\section{Pacific Northwest}

National Laboratory

Operated by Battelle for the

U.S. Department of Energy

\title{
Hanford Area 2000 Population
}

\author{
D.B. Elliott \\ E.J. Antonio \\ M.J. Scott \\ K. Rhoads
}

May 2004

Prepared for the U.S. Department of Energy

under Contract DE-AC06-76RL01830

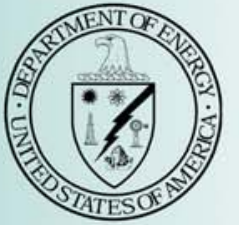


This report was prepared as an account of work sponsored by an agency of the United States Government. Neither the United States Government nor any agency thereof, nor Battelle Memorial Institute, nor any of their employees, makes any warranty, express or implied, or assumes any legal liability or responsibility for the accuracy, completeness, or usefulness of any information, apparatus, product, or process disclosed, or represents that its use would not infringe privately owned rights. Reference herein to any specific commercial product, process, or service by trade name, trademark, manufacturer, or otherwise does not necessarily constitute or imply its endorsement, recommendation, or favoring by the United States Government or any agency thereof, or Battelle Memorial Institute. The views and opinions of authors expressed herein do not necessarily state or reflect those of the United States Government or any agency thereof.

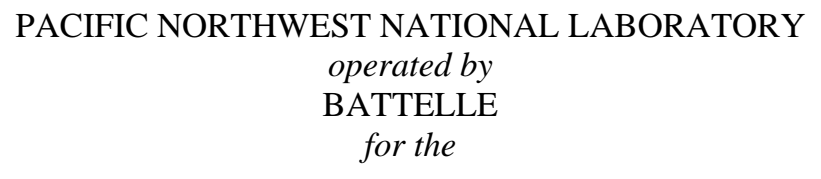

UNITED STATES DEPARTMENT OF ENERGY

under Contract DE-AC06-76RL01830

Printed in the United States of America

\author{
Available to DOE and DOE contractors from the \\ Office of Scientific and Technical Information, \\ P.O. Box 62, Oak Ridge, TN 37831-0062; \\ ph: (865) 576-8401 \\ fax: (865) 576-5728 \\ email: reports@adonis.osti.gov
}

Available to the public from the National Technical Information Service, U.S. Department of Commerce, 5285 Port Royal Rd., Springfield, VA 22161

ph: (800) 553-6847

fax: (703) 605-6900

email: orders@ntis.fedworld.gov

online ordering: http://www.ntis.gov/ordering.htm 
PNNL-14428

\title{
Hanford Area 2000 Population
}

\author{
D.B. Elliott \\ M.J. Scott \\ E.J. Antonio \\ K. Rhoads
}

May 2004

Prepared for

the U.S. Department of Energy

under Contract DE-AC06-76RL01830

Pacific Northwest National Laboratory

Richland, Washington 99352 


\section{Summary}

This report was prepared for the U.S. Department of Energy (DOE) Richland Operations Office, Surface Environmental Surveillance Project, to provide demographic data required for ongoing environmental assessments and safety analyses at the DOE Hanford Site near Richland, Washington. This document includes 2000 Census estimates for the resident population within an 80-kilometer (50mile) radius of the Hanford Site. Population distributions are reported relative to five reference points centered on meteorological stations within major operating areas of the Hanford Site - the $100 \mathrm{~F}, 100 \mathrm{~K}$, 200, 300, and 400 Areas. These data are presented in both graphical and tabular format, and are provided for total populations residing within $80 \mathrm{~km}(50 \mathrm{mi})$ of the reference points, as well as for Native American, Hispanic and Latino, total minority, and low-income populations. 


\section{Contents}

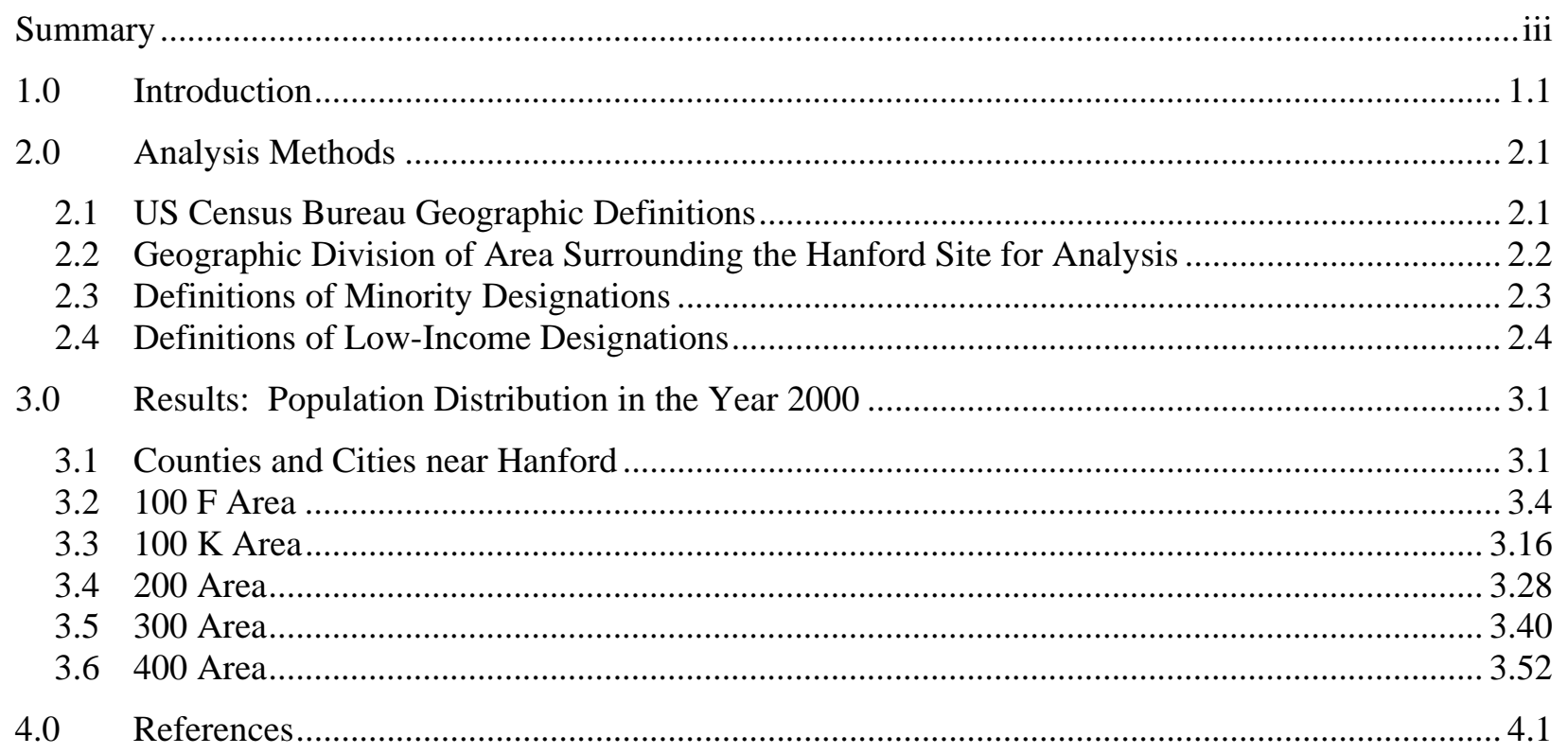




\section{Figures}

1.1. Reference Locations on the Hanford Site

2.1. Map of Census Blocks in Southeast Washington including the Hanford Site with Site Boundary and a 50-Mile Grid Centered on the 200 Area Hanford Meteorological Station

3.1. 2000 Census Populations for Counties and Selected Cities within an 80-km (50-mile) Radius of the Hanford Meteorological Station.

3.2. Map of 80-km (50-mi) Region Surrounding the $100 \mathrm{~F}$ Area Meteorological Station

3.3. Map of Total Resident Population within $80 \mathrm{~km}$ (50 mi) of the Hanford 100 F Area Meteorological Station, in 16-km (10-mi) Increments by Distance and Direction

3.4. Map of Native American Population within $80 \mathrm{~km}$ (50 mi) of the Hanford $100 \mathrm{~F}$ Area Meteorological Station, in 16-km (10-mi) Increments by Distance and Direction...

3.5. Map of Hispanic and Latino Population within $80 \mathrm{~km}$ (50 mi) of the Hanford $100 \mathrm{~F}$ Area Meteorological Station, in 16-km (10-mi) Increments by Distance and Direction.....

3.6. Map of Total Minority Population within $80 \mathrm{~km}$ (50 mi) of the Hanford $100 \mathrm{~F}$ Area Meteorological Station, in 16-km (10-mi) Increments by Distance and Direction...

3.7. Map of Low-Income Population within $80 \mathrm{~km}$ (50 mi) of the Hanford 100 F Area Meteorological Station, in 16-km (10-mi) Increments by Distance and Direction.

3.8. Map of 80-km (50-mi) Region Surrounding the $100 \mathrm{~K}$ Area Meteorological Station.

3.9. Map of Total Resident Population within $80 \mathrm{~km}$ (50 mi) of the Hanford $100 \mathrm{~K}$ Area Meteorological Station, in 16-km (10-mi) Increments by Distance and Direction

3.10. Map of Native American Population within $80 \mathrm{~km}$ (50 mi) of the Hanford $100 \mathrm{~K}$ Area Meteorological Station, in 16-km (10-mi) Increments by Distance and Direction .

3.11. Map of Hispanic and Latino Population within $80 \mathrm{~km}$ (50 mi) of the Hanford $100 \mathrm{~K}$ Area Meteorological Station, in 16-km (10-mi) Increments by Distance and Direction.

3.12. Map of Total Minority Population within $80 \mathrm{~km}$ (50 mi) of the Hanford $100 \mathrm{~K}$ Area Meteorological Station, in 16-km (10-mi) Increments by Distance and Direction .

3.13. Map of Low-Income Population within $80 \mathrm{~km}$ (50 mi) of the Hanford $100 \mathrm{~K}$ Area Meteorological Station, in 16-km (10-mi) Increments by Distance and Direction

3.14. Map of 80-km (50-mi) Region Surrounding the 200 Area Hanford Meteorological Station

3.15. Map of Total Resident Population within $80 \mathrm{~km}$ (50 mi) of the 200 Area Hanford Meteorological Station, in 16-km (10-mi) Increments by Distance and Direction .

3.16. Map of Native American Population within $80 \mathrm{~km}$ (50 mi) of the 200 Area Hanford Meteorological Station, in 16-km (10-mi) Increments by Distance and Direction

3.17. Map of Hispanic and Latino Population within $80 \mathrm{~km}$ (50 mi) of the 200 Area Hanford Meteorological Station, in 16-km (10-mi) Increments by Distance and Direction .

3.18. Map of Total Minority Population within $80 \mathrm{~km}(50 \mathrm{mi})$ of the 200 Area Hanford Meteorological Station, in 16-km (10-mi) Increments by Distance and Direction

3.19. Map of Low-Income Population within $80 \mathrm{~km}$ (50 mi) of the 200 Area Hanford Meteorological Station, in 16-km (10-mi) Increments by Distance and Direction 
3.20. Map of 80-km (50-mi) Region Surrounding the 300 Area Meteorological Station.

3.21. Map of Total Resident Population within $80 \mathrm{~km}$ (50 mi) of the Hanford 300 Area Meteorological Station, in 16-km (10-mi) Increments by Distance and Direction

3.22. Map of Native American Population within $80 \mathrm{~km}(50 \mathrm{mi})$ of the Hanford 300 Area Meteorological Station, in 16-km (10-mi) Increments by Distance and Direction.....

3.23. Map of Hispanic and Latino Population within $80 \mathrm{~km}(50 \mathrm{mi})$ of the Hanford 300 Area Meteorological Station, in 16-km (10-mi) Increments by Distance and Direction

3.24. Map of Total Minority Population within $80 \mathrm{~km}$ (50 mi) of the Hanford 300 Area Meteorological Station, in 16-km (10-mi) Increments by Distance and Direction

3.25. Map of Low-Income Population within $80 \mathrm{~km}(50 \mathrm{mi})$ of the Hanford 300 Area Meteorological Station, in 16-km (10-mi) Increments by Distance and Direction

3.26. Map of 80-km (50-mi) Region Surrounding the 400 Area Meteorological Station

3.27. Map of Total Resident Population within $80 \mathrm{~km}$ (50 mi) of the Hanford 400 Area Meteorological Station, in 16-km (10-mi) Increments by Distance and Direction

3.28. Map of Native American Population within $80 \mathrm{~km}(50 \mathrm{mi})$ of the Hanford 400 Area Meteorological Station, in 16-km (10-mi) Increments by Distance and Direction

3.29. Map of Hispanic and Latino Population within $80 \mathrm{~km}(50 \mathrm{mi})$ of the Hanford 400 Area Meteorological Station, in 16-km (10-mi) Increments by Distance and Direction

3.30. Map of Total Minority Population within $80 \mathrm{~km}$ (50 mi) of the Hanford 400 Area Meteorological Station, in 16-km (10-mi) Increments by Distance and Direction

3.31. Map of Low-Income Population within $80 \mathrm{~km}(50 \mathrm{mi})$ of the Hanford 400 Area Meteorological Station, in 16-km (10-mi) Increments by Distance and Direction 


\section{Tables}

3.1. Population Growth from 1990 to 2000 in Selected Counties and Cities Surrounding the Hanford Site

3.2. Distribution of Total Resident Population within $80 \mathrm{~km}$ (50 mi) of the Hanford 100 F Area Meteorological Station.

3.3. Distribution of Native American Population within $80 \mathrm{~km}$ (50 mi) of the Hanford 100 F Area Meteorological Station.

3.4. Distribution of Hispanic and Latino Population within $80 \mathrm{~km}$ (50 mi) of the Hanford 100 F Area Meteorological Station.

3.5. Distribution of Total Minority Population within $80 \mathrm{~km}$ (50 mi) of the Hanford 100 F Area Meteorological Station.

3.6. Distribution of Low-Income Population within $80 \mathrm{~km}$ (50 mi) of the Hanford 100 F Area Meteorological Station.

3.7. Distribution of Total Resident Population within $80 \mathrm{~km}$ (50 mi) of the Hanford $100 \mathrm{~K}$ Area Meteorological Station

3.8. Distribution of Native American Population within $80 \mathrm{~km}$ (50 mi) of the Hanford $100 \mathrm{~K}$ Area Meteorological Station

3.9. Distribution of Hispanic and Latino Population within $80 \mathrm{~km}$ (50 mi) of the Hanford 100 K Area Meteorological Station

3.10. Distribution of Total Minority Population within $80 \mathrm{~km}$ (50 mi) of the Hanford $100 \mathrm{~K}$ Area Meteorological Station

3.11. Distribution of Low-Income Population within $80 \mathrm{~km}$ (50 mi) of the Hanford $100 \mathrm{~K}$ Area Meteorological Station

3.12. Distribution of Total Resident Population within $80 \mathrm{~km}$ (50 mi) of the 200 Area Hanford Meteorological Station

3.13. Distribution of Native American Population within $80 \mathrm{~km}$ (50 mi) of the 200 Area Hanford Meteorological Station

3.14. Distribution of Hispanic and Latino Population within $80 \mathrm{~km}$ (50 mi) of the 200 Area Hanford Meteorological Station

3.15. Distribution of Total Minority Population within $80 \mathrm{~km}$ (50 mi) of the 200 Area Hanford Meteorological Station

3.16. Distribution of Low-Income Population within $80 \mathrm{~km}$ (50 mi) of the 200 Area Hanford Meteorological Station

3.17. Distribution of Total Resident Population within $80 \mathrm{~km}$ (50 mi) of the Hanford 300 Area Meteorological Station

3.18. Distribution of Native American Population within $80 \mathrm{~km}$ (50 mi) of the Hanford 300 Area Meteorological Station

3.19. Distribution of Hispanic and Latino Population within $80 \mathrm{~km}$ (50 mi) of the Hanford 300 Area Meteorological Station 
3.20. Distribution of Total Minority Population within $80 \mathrm{~km}$ (50 mi) of the Hanford 300 Area Meteorological Station

3.21. Distribution of Low-Income Population within $80 \mathrm{~km}$ (50 mi) of the Hanford 300 Area Meteorological Station

3.22. Distribution of Total Resident Population within $80 \mathrm{~km}$ (50 mi) of the Hanford 400 Area Meteorological Station

3.23. Distribution of Native American Population within $80 \mathrm{~km}$ (50 mi) of the Hanford 400 Area Meteorological Station

3.24. Distribution of Hispanic and Latino Population within $80 \mathrm{~km}$ (50 mi) of the Hanford 400 Area Meteorological Station

3.25. Distribution of Total Minority Population within $80 \mathrm{~km}$ (50 mi) of the Hanford 400 Area Meteorological Station

3.26. Distribution of Low-Income Population within $80 \mathrm{~km}$ (50 mi) of the Hanford 400 Area Meteorological Station 


\subsection{Introduction}

Population size and distribution are important criteria for assessing the magnitude of the risk to the public from radionuclide and hazardous chemical emissions, whether from accidents or in the course of normal operations. Data pertaining to population size and distribution are required to prepare environmental dose assessments for radionuclide emissions as required by DOE Order 5400.5 (DOE 1993) and other types of risk evaluations prepared under the National Environmental Policy Act (42 USC 4321) or other requirements. They may also be used by non-DOE facilities within the Hanford Site to ensure that nonreactor nuclear facility Safety Analysis Reports are in compliance with U.S. Nuclear Regulatory Commission (NRC) guidelines (NRC 1975; NRC 1988).

Previous population estimates were prepared for the Hanford Site based on the 1980 Census (Sommer et al. 1981) and the 1990 Census of Population (Beck et al. 1991). This report was prepared based on 2000 Census data to provide updated information for the Hanford Site. The report presents total population estimates within an 80-km (50-mile) radius from five reference points on the Hanford Site: the 100 F Area Meteorological Station, the 100 K Area Meteorological Station, the 200 Area Hanford Meteorological Station (Building 622-R), the 400 Area Meteorological Station, and the 300 Area Meteorological Station (Building 303C) (see Figure 1.1). The areas within $80 \mathrm{~km}$ (50 mi) of these reference locations encompass all or part of twelve counties in two states (see Figure 3.2). The Washington counties include Adams, Benton, Franklin, Grant, Kittitas, Klickitat, Walla Walla, and Yakima. The Oregon counties include Morrow and Umatilla. The 100 Area radii also include very small portions of Chelan and Douglas Counties in Washington. The 50-year forecasts in Sommer et al. (1981) and Beck et al. (1991) were very seldom used, so updated 50-year forecasts were not prepared for this report.

In February 1994, Executive Order 12898, "Federal Actions to Address Environmental Justice in Minority Populations and Low-Income Populations” (59 FR 7629), directed federal agencies in the Executive Branch to consider environmental justice so that their programs would not have “disproportionately high and adverse human health or environmental effects" on minority and lowincome populations. The Council on Environmental Quality (CEQ) later provided additional guidance for integrating environmental justice (EJ) into the National Environmental Policy Act process in a December 1997 document, Environmental Justice Guidance under the National Environmental Policy Act (CEQ 1997). In response to those guidelines, this report identifies minority and low-income populations within $80 \mathrm{~km}$ (50 mi) of the five reference points. Population estimates include total resident, total minority, Hispanic and Latino, Native American, and low-income populations. 


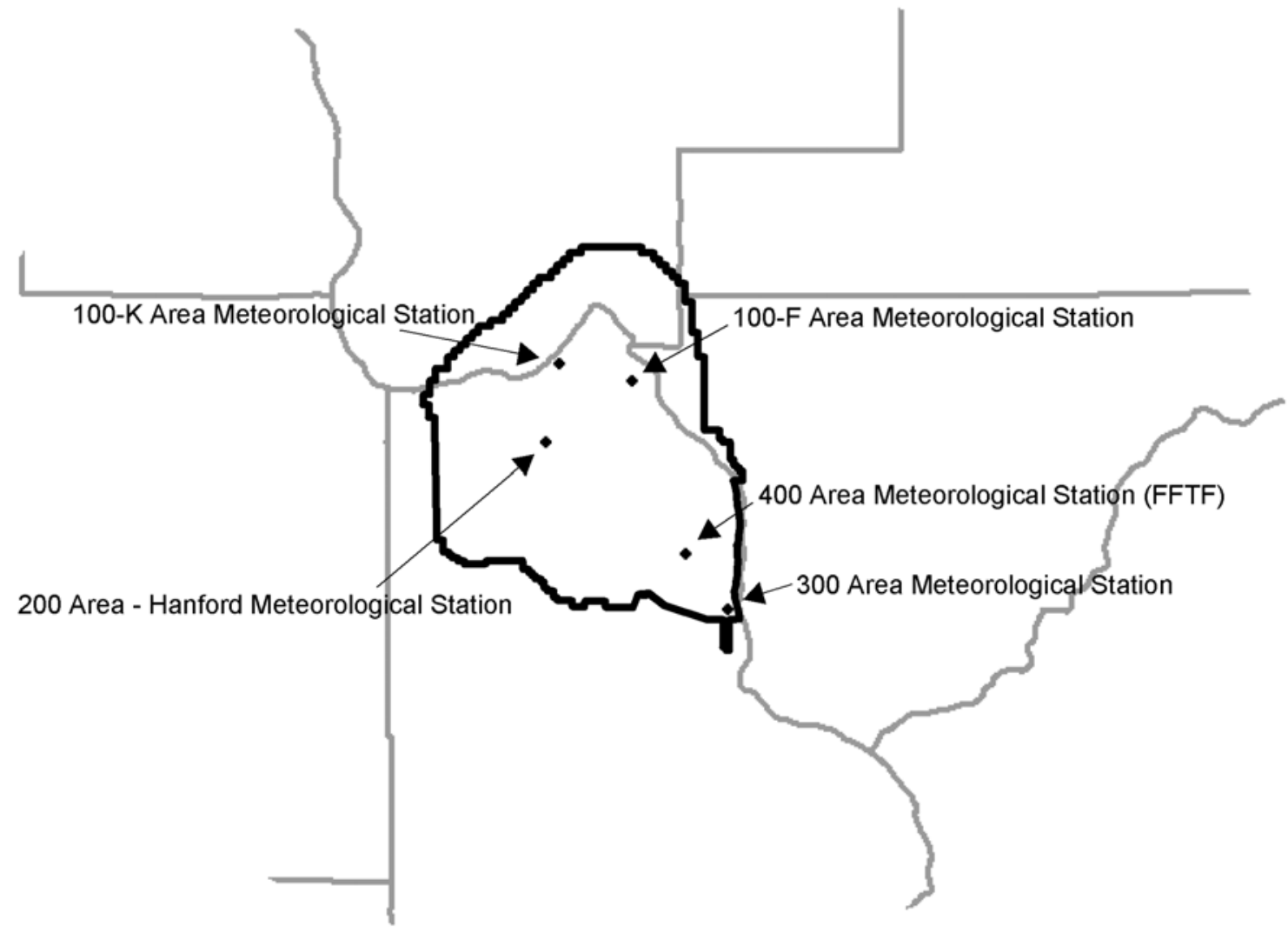

Figure 1.1. Reference Locations on the Hanford Site 


\subsection{Analysis Methods}

The analyses for this report used a methodology similar to that in prior analyses of Hanford area population conducted with 1990 Census data (Beck et al. 1991). However, due to advances in geographic information systems and mapping tools, this report provides greater resolution and detail than the 1991 study, which presented data in 10-mile increments from the Hanford reference points. This report also uses census data at the block and block group level rather than at the larger tract level, which locates populations with greater accuracy and precision. Another change from the Beck et al. (1991) report was the choice of reference points. Four reference points were used in that report, including the $100 \mathrm{~N}, 200$, 300, and 400 Areas. For this report the $100 \mathrm{~N}$ Area reference point was replaced by two different 100 Area points - the $100 \mathrm{~K}$ and $100 \mathrm{~F}$ Areas - to better reflect current site cleanup activities. The reactor at the $100 \mathrm{~N}$ Area has been shut down and decommissioning of the facilities at that site is well underway. The $100 \mathrm{~K}$ and $100 \mathrm{~F}$ Area population distributions were included in this report to provide representative data for ongoing remediation activities at a number of nearby sites along the Columbia River corridor.

The population distributions developed for this report are presented as circular grids by distance and direction relative to the five reference points identified previously. In contrast, the US Census geographical unit allocations are based on population and have irregular boundaries that are established with assistance from local committees. The process for distributing population data gathered from census units into the circular grid used for Hanford Site assessments is described in the following sections. Figure 2.1 shows a map of census blocks in southeast Washington relative to the Hanford Site boundary and an 80-km (50-mile) circular grid centered on the 200 Area Hanford Meteorological Station.

\subsection{US Census Bureau Geographic Definitions}

The U.S. Census Bureau divides counties into tracts, block groups, and blocks. Census tracts are small, relatively permanent, statistical subdivisions of counties delineated by local committees of census data users in accordance with Census Bureau guidelines for the purpose of collecting and presenting decennial census data. Census tracts generally contain between 1,000 and 8,000 people, with an optimum size of 4,000 people (Census 2003a). Census tracts are composed of block groups. A block group contains one or more census blocks and is the lowest-level geographic entity for which the Census Bureau tabulates sample data (such as income) from the decennial census. A census block is the smallest geographic entity for which the U.S. Census Bureau collects and tabulates 100 percent decennial census population count information (such as race and ethnicity).

Among the 367 census block groups within 50 miles of the 200 Area Hanford Meteorological Station, the largest had a population of 5,649 individuals, and the smallest had a population of zero. There are 17,833 census blocks within or partially within the 80 -km (50-mile) radius grid relative to the Hanford Meteorological Station. The block with the largest population contains 1,747 people, and there are 7,129 blocks with no residents. 


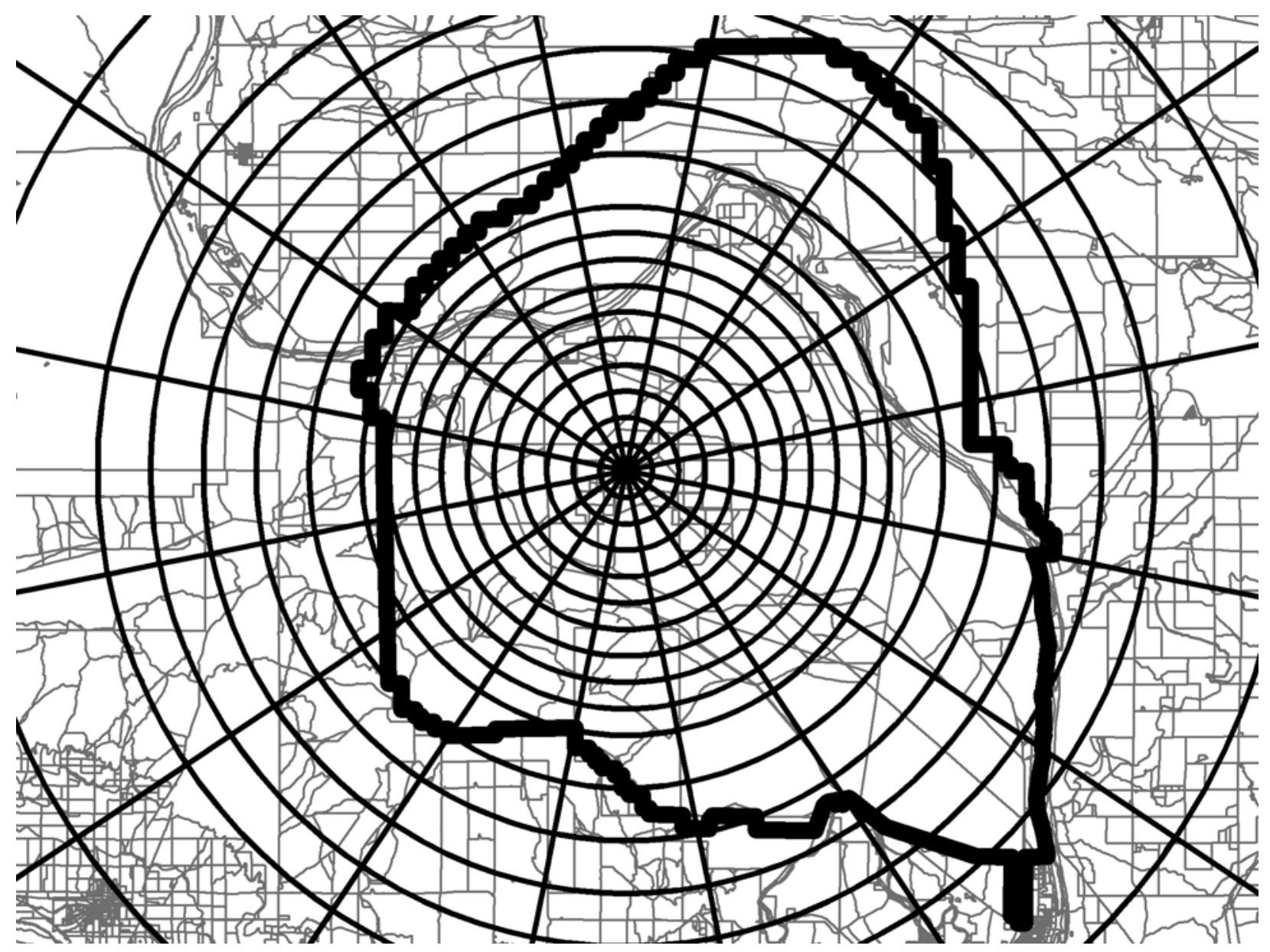

Figure 2.1. Map of Census Blocks in Southeast Washington including the Hanford Site with Site Boundary and a 50-Mile Grid Centered on the 200 Area Hanford Meteorological Station. Grid is shown out to approximately 25 miles.

\subsection{Geographic Division of Area Surrounding the Hanford Site for Analysis}

The population data contained in this report are distributed into circular grids relative to each of the five identified reference points on the Hanford Site. Each grid consists of sectors defined by circles of 20 unique radii (i.e., distances from the reference point), with the first 0 to 10 miles being in 1-mile increments, 10 to 20 miles in 2-mile increments, 20 to 40 miles in 5-mile increments, and 40 to 50 miles in one increment. The 20 annular areas defined by these circles are divided into 16 radial sectors corresponding to the major compass directions, creating a 320-sector circular grid (partially shown in Figure 2.1). These 320-sector distance-and-direction grids were centered on each of the five reference points.

The populations within each of the 320 sectors of these circular grids were determined in two steps. First, for maximum accuracy, the population totals were extracted from 2000 Census population data at the census block level (for total population and minorities) (Census 2001a and b) and at the block group 
level (Census 2002a and b) for low-income populations, along with geographic information that referenced the population data to a map of the area (Census 2001c). To ensure consistency and comparability with the block-level racial data, the percentages of low-income population at the block group-level were multiplied by the total population in each of the block group's constituent blocks. This calculated block-level low-income population was then allocated among the 320 sectors.

Second, the populations were placed into a geographic information system (GIS) software package to produce population estimates for each sector in the circular grid. Because of the irregular shape and size of the Census blocks, it was often necessary to allocate parts of the census block population into one or more sectors of the circular grid. This was required where census blocks fell into two or more sectors (see Figure 2.1), or where blocks located along the outer edge of the grid were only partially contained within the grid. In such cases, a fraction of the census block population was assigned to each grid sector based on the fraction of the census block area contained within that sector using the "geoprocessing" capabilities within the GIS software.

In order to assign the census block populations to the appropriate grid sectors, the total area of each of the blocks was calculated. The blocks were then divided into segments that fell within each of the grid sectors, and the area of the resulting pieces was calculated. The census block segments were assigned populations in proportion to their resulting area, using the implicit assumption of a uniform distribution of population within each block. Thus, if a grid cell contained $27 \%$ of the land area of a particular census block, it also was assumed to contain $27 \%$ of the block's population. To ensure that the sum of the populations for all 320 sectors matched that for the entire 50-mile grid, rounding of any resulting fractional populations was avoided during this process until the final presentation of results.

Using this process in combination with the most finely-detailed census data provides the greatest accuracy in the population distributions; however, any adjustment of this type involves a degree of unavoidable uncertainty in allocating the block population. Where census blocks are geographically large and the population distributions within the block are less uniform, this procedure can lead to larger uncertainties in assigning population. However, for the most part, any errors introduced by this process are small and usually reassign small numbers of people to neighboring cells.

\subsection{Definitions of Minority Designations}

The "minorities" designation in the Census data counts both racial minorities (black, Asian, Native American, Alaska Native, Pacific islander, and either mixed race or “other” race), and Hispanics and Latinos. The Hispanic and Latino category may be viewed as the ancestry, nationality group, lineage, or country of birth of the person or person's parents or ancestors before arrival in the United States. Hispanics and Latinos may be of any race, so white Hispanics and Latinos are added to the racial minorities (which includes non-white Hispanics and Latinos) to obtain an estimate of the total minority population. Nationally, in 2000, non-Hispanic or non-Latino whites were $69.1 \%$ of the population, meaning that minorities were $30.9 \%$ (Census 2001d). 


\subsection{Definitions of Low-Income Designations}

Low-income individuals live in households that report an annual income less than the United States official poverty level, as reported by the Census Bureau. The poverty level varies by size and relationship of the members of the household. The 2000 Census poverty level was based on incomes in the year 1999 and was set at $\$ 17,029$ for a family of four in that year (Census 2003b). Nationally, in 1999, 12.4\% of all persons lived in households that had incomes below the poverty level. 


\subsection{Results: Population Distribution in the Year 2000}

Population estimates for each population group in each of the areas surrounding the five reference points are presented in tables, as well as graphically, in the following sections. The tables are presented as matrices of 320 sectors composed of population estimates at each of the 20 radii (20 incremental distances from the reference points) for each of the 16 standard compass-point directions. The graphic presentation consists of circular distance-and-direction grids centered on each of the five reference points. The graphic information has been aggregated into an 80-sector grid to simplify viewing. The grids show populations at five 16-km (10-mile) intervals from the reference points, again divided into 16 compass directions.

Section 3.1 discusses population growth in the counties and cities near Hanford from 1990 to 2000. Sections 3.2 through 3.6 provide the graphic distance and direction grids and 320-sector tables for populations within $80 \mathrm{~km}$ (50 miles) of the five reference points on the Hanford Site: the $100 \mathrm{~F}$ Area Meteorological Station, the $100 \mathrm{~K}$ Area Meteorological Station, the Hanford Meteorological Station in the 200 Area, the 300 Area Meteorological Station, and the 400 Area Meteorological Station. For each reference point, five sets of tables and grids are provided for total populations as well as for minority and low-income populations. There are currently no permanent residents within the Hanford Site boundary.

\subsection{Counties and Cities near Hanford}

Cities closest to the Hanford reservation include Richland, Kennewick, Pasco, West Richland, Benton City, Prosser, Sunnyside, Grandview, and Mesa, all of which are located in southeastern Washington State. The locations and year 2000 populations for these and other nearby communities are presented in Figure 3.1. The closest major city to any Hanford facility is Richland, Washington, which is located in Benton County and had a population of 38,708 according to the 2000 census.

During the decade of the 1990s, the population of Benton and Franklin Counties, where most Hanford employees reside, grew at a compounded annual rate of $2.5 \%$, while the 10 counties surrounding the site as a whole grew at $2.1 \%$ per year. For comparison, the nation's population growth rate was about $1.2 \%$ per year from 1990 to 2000. The more rapid local growth rate occurred in part due to the increased funding devoted to Hanford cleanup and the development of the local economy. Table 3.1 shows the growth for selected counties, towns, and cities within the region surrounding the Hanford Site. Many cities and towns in the area grew by a third or more during the decade, and four of them more than doubled in size. 


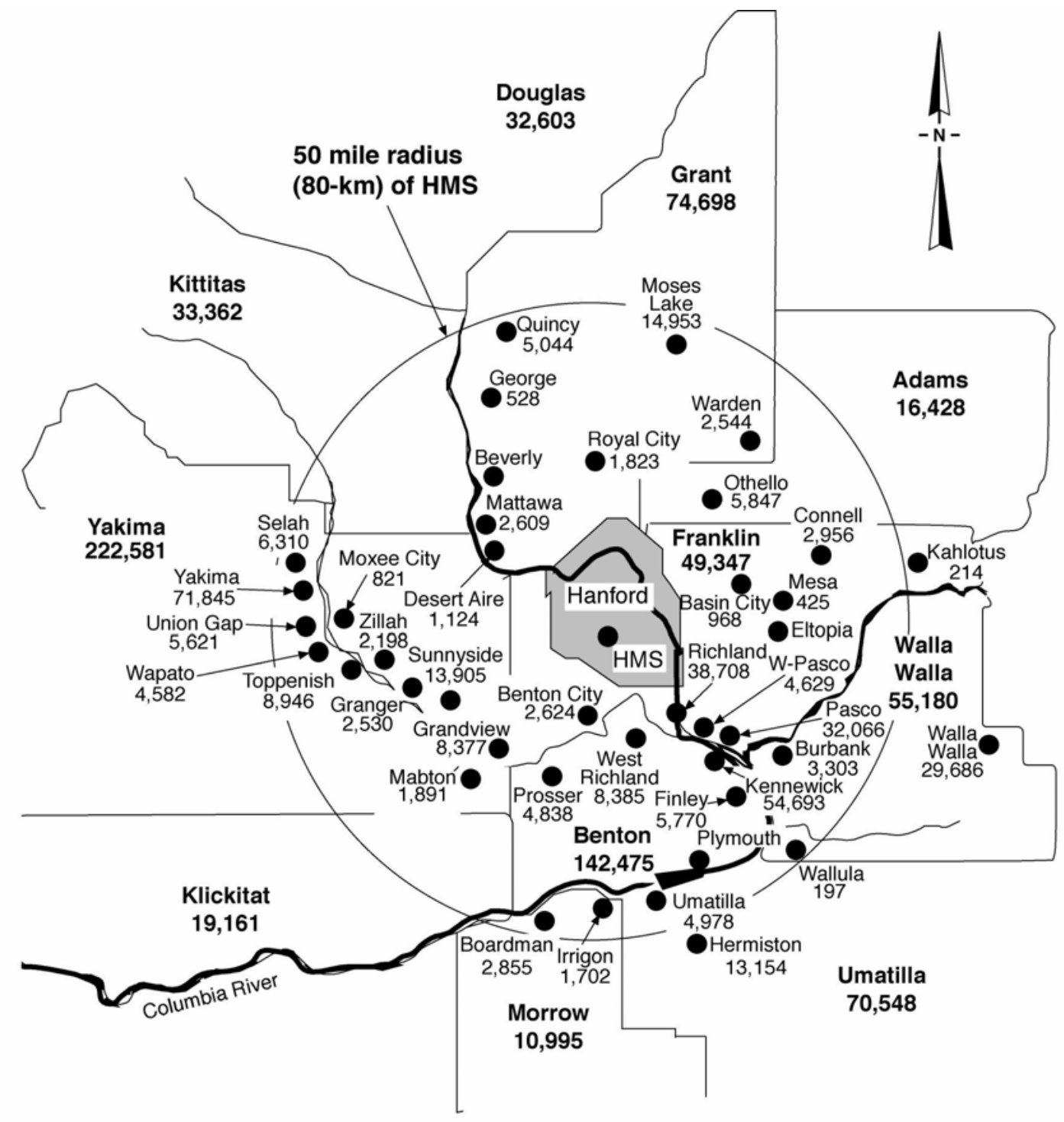

Note: If population not listed, less than 1,000.

HMS = Hanford Meteorological Station

Figure 3.1. 2000 Census Populations for Counties and Selected Cities within an 80-km (50-mile) Radius of the Hanford Meteorological Station 
Table 3.1. Population Growth from 1990 to 2000 in Selected Counties and Cities Surrounding the Hanford Site

\begin{tabular}{|c|c|c|c|c|c|c|c|}
\hline & Census & Census & $\begin{array}{l}\text { Percent } \\
\text { Growth }\end{array}$ & & Census & Census & $\begin{array}{l}\text { Percent } \\
\text { Growth }\end{array}$ \\
\hline Washington & 1990 & 2000 & $1990-2000$ & Washington, Cont. & 1990 & 2000 & $\begin{array}{r}1990- \\
2000 \\
\end{array}$ \\
\hline Adams County & 13,603 & 16,428 & $20.8 \%$ & Walla Walla County & 48,439 & 55,180 & $13.9 \%$ \\
\hline \multirow[t]{3}{*}{ Othello } & 4,638 & 5,847 & $26.1 \%$ & Burbank & 1,745 & 3,303 & $89.3 \%$ \\
\hline & & & & Walla Walla & 26,482 & 29,686 & $12.1 \%$ \\
\hline & & & & Wallula & N/A & 197 & N/A \\
\hline Benton County & 112,560 & 142,475 & $26.6 \%$ & Yakima County & 188,823 & 222,581 & $17.9 \%$ \\
\hline Benton City & 1,806 & 2,624 & $45.3 \%$ & Grandview & 7,169 & 8,377 & $16.9 \%$ \\
\hline Finley & 4,897 & 5,770 & $17.8 \%$ & Granger & 2,053 & 2,530 & $23.2 \%$ \\
\hline Kennewick & 42,152 & 54,751 & $29.9 \%$ & Mabton & 1,482 & 1,891 & $27.6 \%$ \\
\hline Prosser & 4,476 & 4,838 & $8.1 \%$ & Moxee & 825 & 821 & $-0.5 \%$ \\
\hline Richland & 32,315 & 38,708 & $19.8 \%$ & Selah & 5,113 & 6,310 & $23.4 \%$ \\
\hline West Richland & 3,962 & 8,385 & $111.6 \%$ & Sunnyside & 11,238 & 13,905 & $23.7 \%$ \\
\hline $\begin{array}{l}\text { Franklin } \\
\text { County }\end{array}$ & 37,473 & 49,347 & $31.7 \%$ & Toppenish & 7,419 & 8,946 & $20.6 \%$ \\
\hline Connell & 2,005 & 2,956 & $47.4 \%$ & Union Gap & 3,120 & 5,621 & $80.2 \%$ \\
\hline Kahlotus & 167 & 214 & $28.1 \%$ & Wapato & 3,795 & 4,582 & $20.7 \%$ \\
\hline Mesa & 252 & 425 & $68.7 \%$ & Yakima & 54,843 & 71,845 & $31.0 \%$ \\
\hline Pasco & 20,337 & 32,066 & $57.7 \%$ & Zillah & 1,911 & 2,198 & $15.0 \%$ \\
\hline Grant County & 54,798 & 74,698 & $36.3 \%$ & Oregon & & & \\
\hline Desert Aire & N/A & 1,124 & N/A & $\overline{\text { Morrow County }}$ & 7,625 & 10,995 & $44.2 \%$ \\
\hline George & 324 & 528 & $63.0 \%$ & Boardman & 1,387 & 2,855 & $105.8 \%$ \\
\hline Mattawa & 941 & 2,609 & $177.3 \%$ & Irrigon & 737 & 1,702 & $130.9 \%$ \\
\hline Moses Lake & 11,235 & 14,953 & $33.1 \%$ & & & & \\
\hline Quincy & 3,738 & 5,044 & $34.9 \%$ & Umatilla County & 59,249 & 70,548 & $19.1 \%$ \\
\hline Royal City & 1,104 & 1,823 & $65.1 \%$ & Hermiston & 10,040 & 13,154 & $31.0 \%$ \\
\hline Warden & 1,639 & 2,544 & $55.2 \%$ & Umatilla & 3,046 & 4,978 & $63.4 \%$ \\
\hline Kittitas County & 26,725 & 33,362 & $24.8 \%$ & $\begin{array}{l}\text { Sum for Counties } \\
\text { Shown }\end{array}$ & 565,911 & 694,775 & $22.8 \%$ \\
\hline $\begin{array}{l}\text { Klickitat } \\
\text { County }\end{array}$ & 16,616 & 19,161 & $15.3 \%$ & & & & \\
\hline & & & & & & & \\
\hline
\end{tabular}




\section{2 $100 \mathrm{~F}$ Area}

The 100 F Area Meteorological Station is near the northeast corner of the Hanford Site. At the 2000 Census there were about 382,200 people residing within $80 \mathrm{~km}$ (50 mi) of this location. Based on census block data, about 149,500 (39\%) of these residents were minorities. The Hispanic and Latino population (all races) was the single largest minority group, with 127,200 members (33\% of the total population). There were 7,500 Native Americans (2\% of the total). There were an estimated 65,800 low-income individuals (about $17 \%$ of the population).

Figures 3.2 to 3.7 and Tables 3.2 to 3.6 show population distributions for total, minority, and lowincome populations living within 50 miles of the $100 \mathrm{~F}$ Area Meteorological Station on the Hanford Site.

About 300 people live within 10 miles of the $100 \mathrm{~F}$ Area Meteorological Station. There are about 3,000 people within 15 miles, and 18,000 people within 20 miles, mostly from the north to ENE. There are about 36,000 people living between 20 and 25 miles from the 100 F Area Meteorological Station, mostly SSE to south. There is a small concentration of Native Americans living between 18 and 20 miles to the north, and a few hundred living in the Tri-Cities area 20-50 miles to the south and southeast. The largest Native American population is 40-50 miles WSW in the Yakima area, with over 4,000 individuals. Hispanic and Latino populations are concentrated 16-20 miles to the northeast (6,300 individuals), 20-25 miles to the WNW (2,900-plus individuals), 25-30 miles SSE (2,300 individuals), 30-35 miles to the SSE (25,400 individuals) and SW (17,300 individuals), 35-40 miles to the SW (6,900 individuals) and 40-50 miles to the WSW (17,800 individuals) and due west (11,600 individuals). 


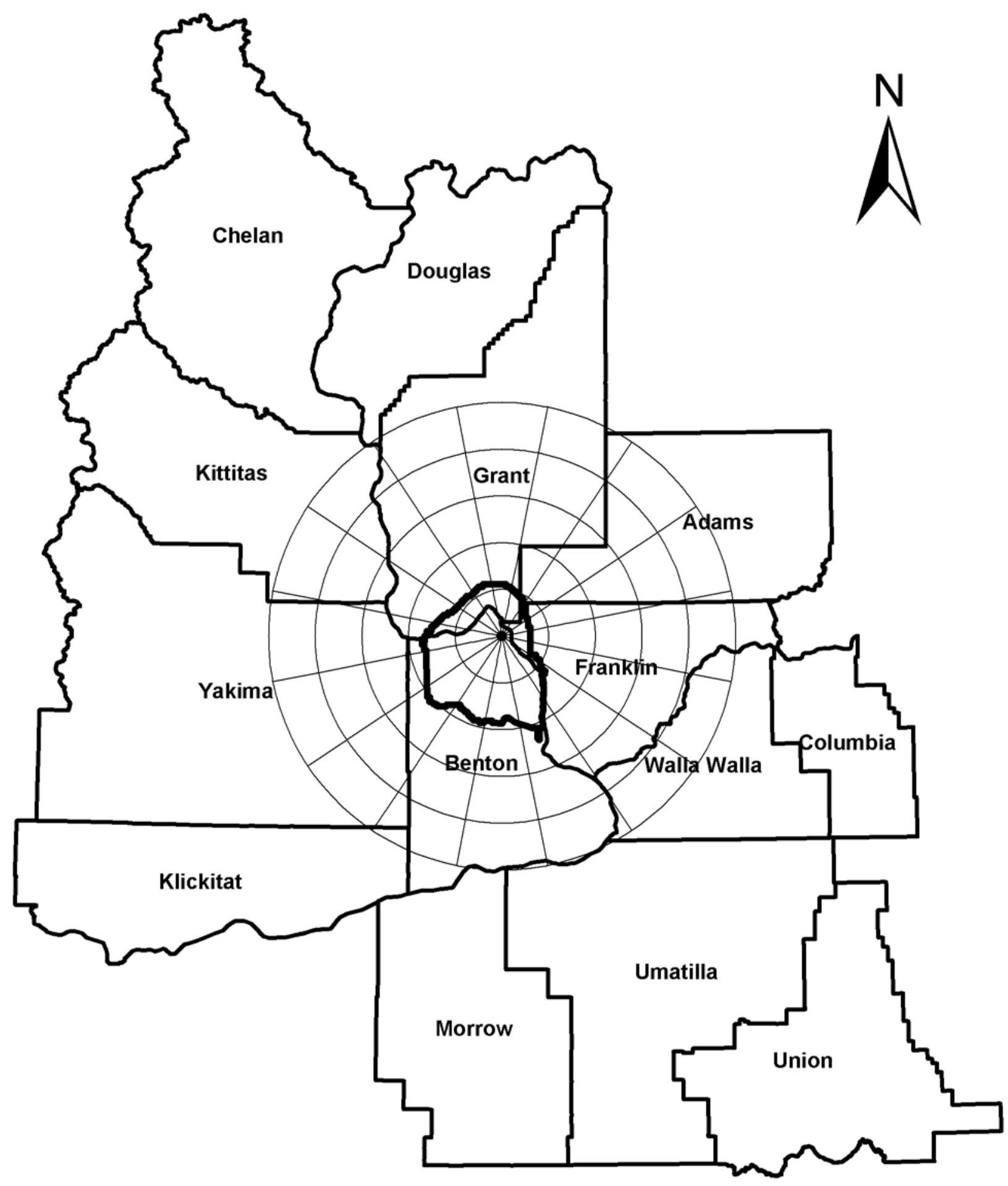

Figure 3.2. Map of 80-km (50-mi) Region Surrounding the $100 \mathrm{~F}$ Area Meteorological Station 


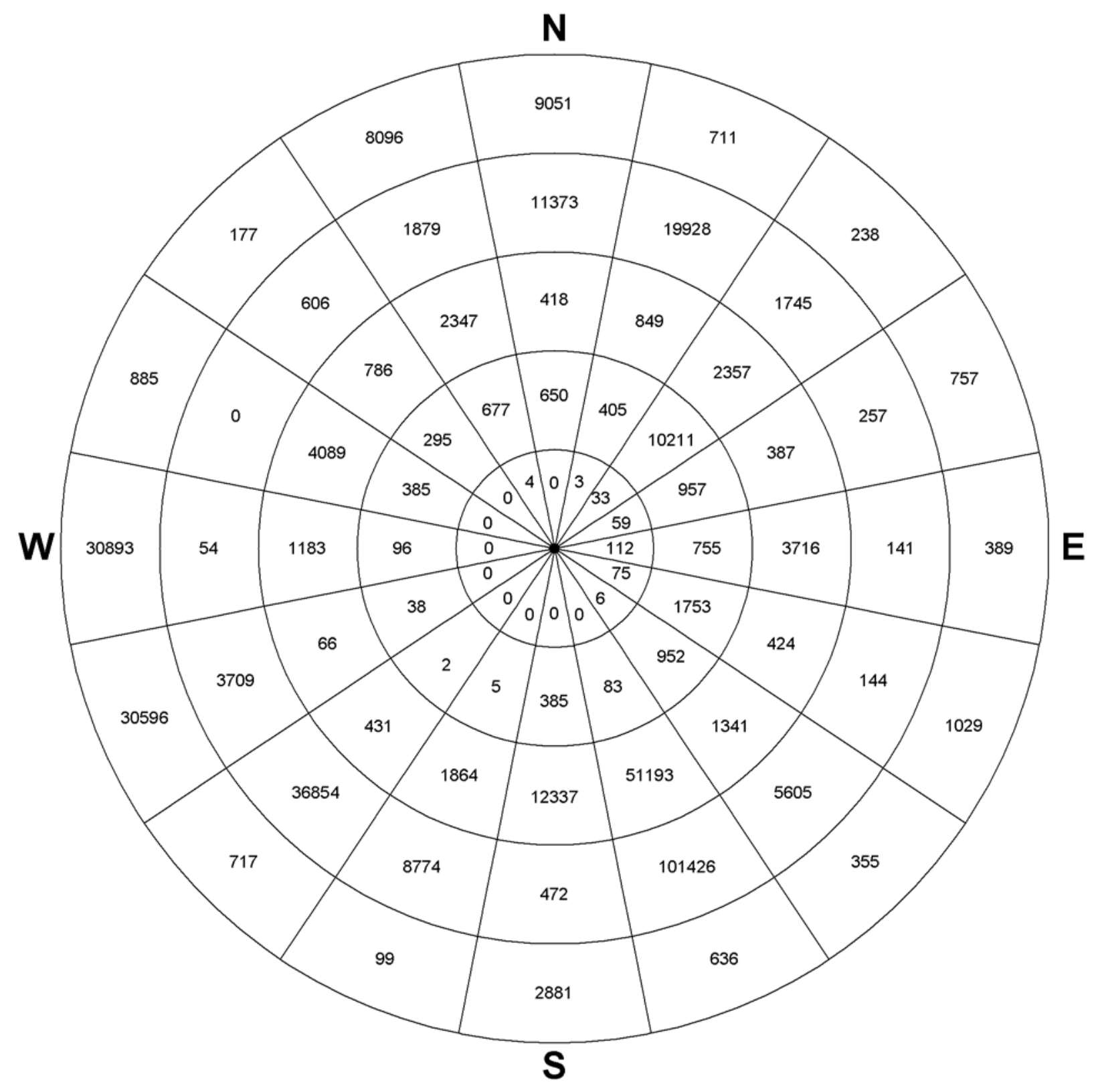

Figure 3.3. Map of Total Resident Population within $80 \mathrm{~km}(50 \mathrm{mi})$ of the Hanford $100 \mathrm{~F}$ Area Meteorological Station, in 16-km (10-mi) Increments by Distance and Direction 


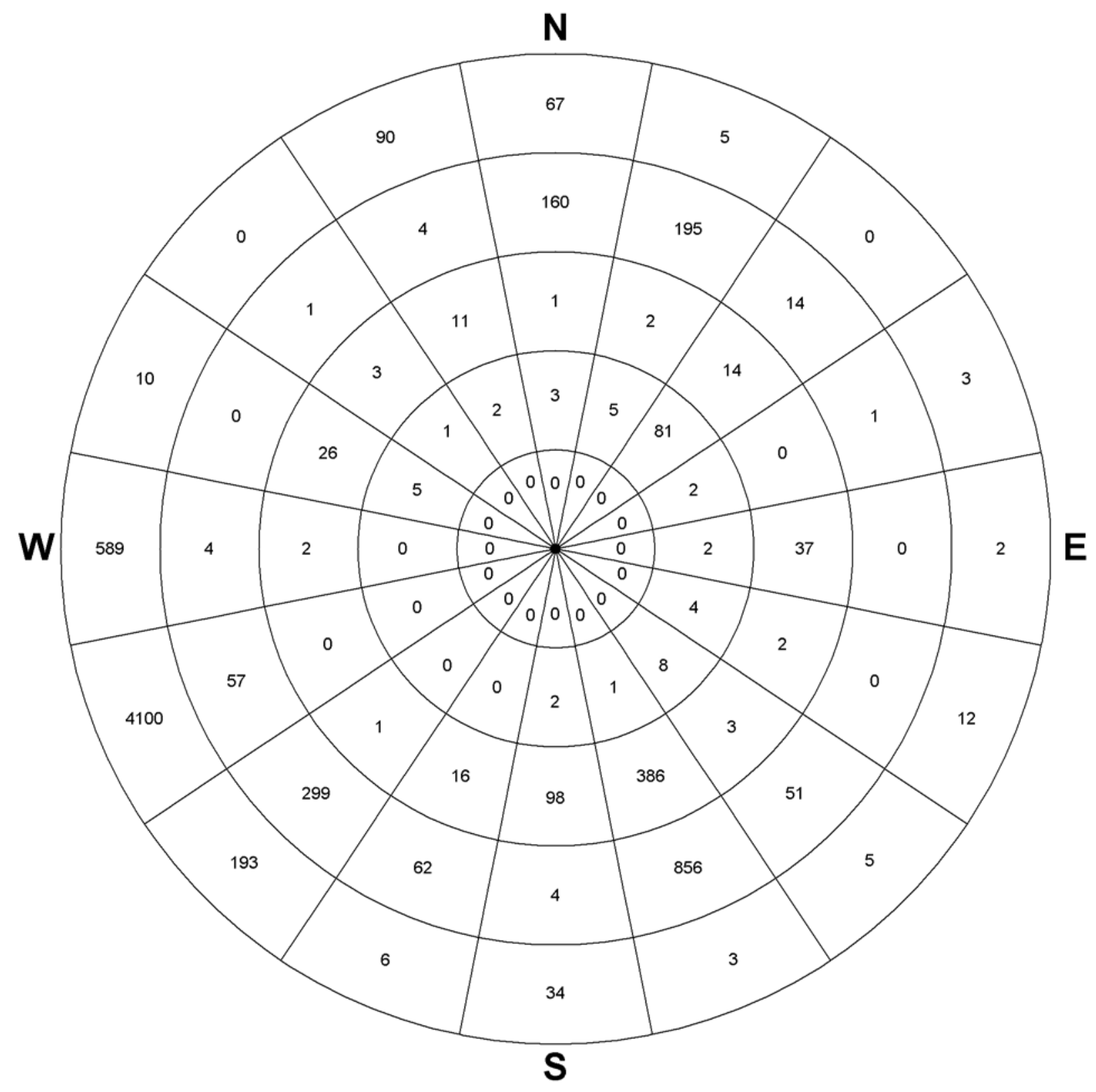

Figure 3.4. Map of Native American Population within $80 \mathrm{~km}$ (50 mi) of the Hanford $100 \mathrm{~F}$ Area Meteorological Station, in 16-km (10-mi) Increments by Distance and Direction 


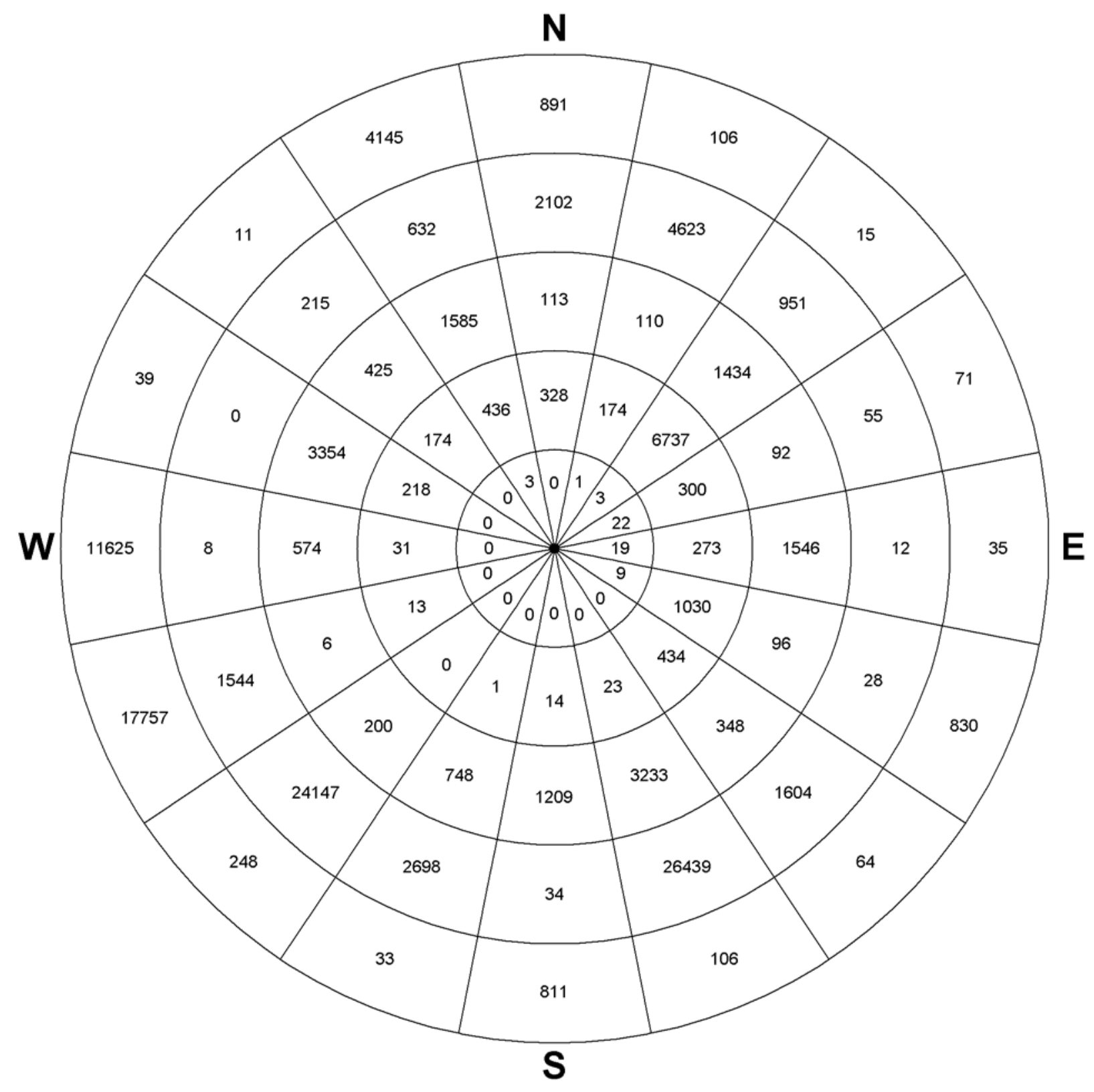

Figure 3.5. Map of Hispanic and Latino Population within $80 \mathrm{~km}(50 \mathrm{mi})$ of the Hanford $100 \mathrm{~F}$ Area Meteorological Station, in 16-km (10-mi) Increments by Distance and Direction 


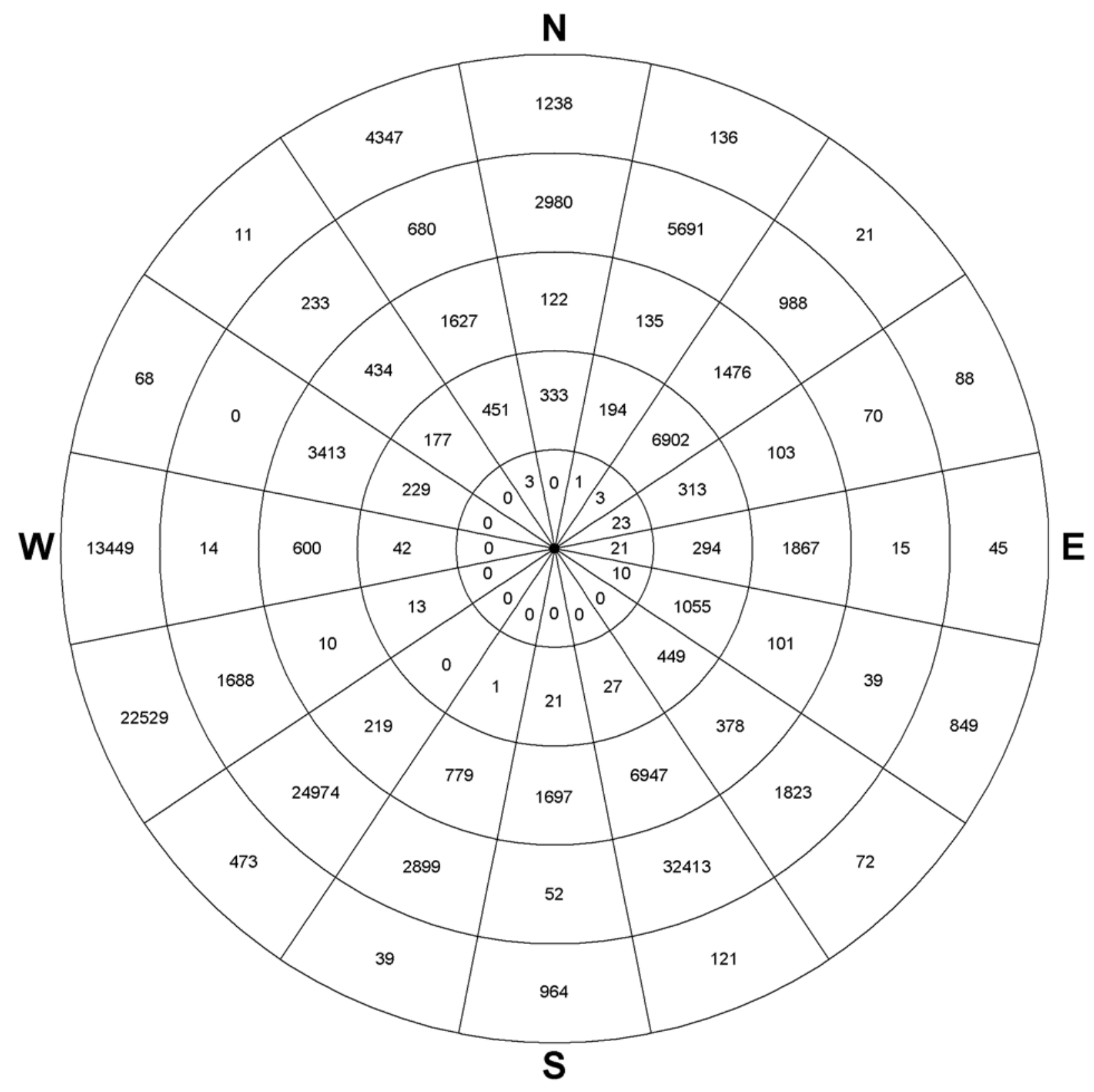

Figure 3.6. Map of Total Minority Population within $80 \mathrm{~km}(50 \mathrm{mi})$ of the Hanford $100 \mathrm{~F}$ Area Meteorological Station, in 16-km (10-mi) Increments by Distance and Direction 


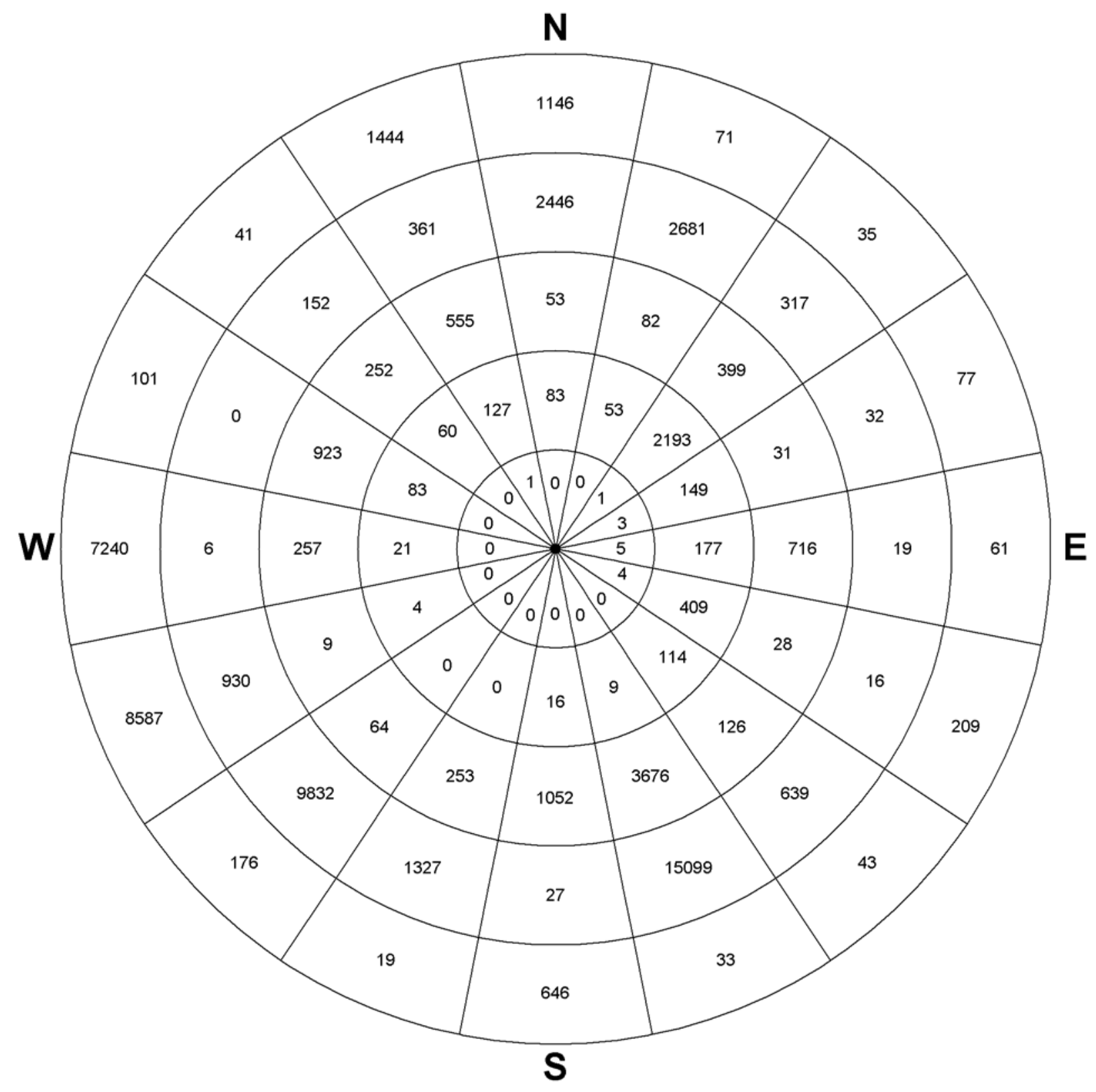

Figure 3.7. Map of Low-Income Population within $80 \mathrm{~km}(50 \mathrm{mi})$ of the Hanford $100 \mathrm{~F}$ Area Meteorological Station, in 16-km (10-mi) Increments by Distance and Direction 
Table 3.2. Distribution of Total Resident Population within 80 km (50 mi) of the Hanford 100 F Area Meteorological Station

\begin{tabular}{|c|c|c|c|c|c|c|c|c|c|c|c|c|c|c|c|c|c|}
\hline & \multicolumn{17}{|c|}{ Direction } \\
\hline $\begin{array}{l}\text { Distance } \\
\text { from } \\
\text { Reference } \\
\text { Point } \\
\text { (Miles) } \\
\end{array}$ & $\mathbf{N}$ & NNE & NE & ENE & $\mathbf{E}$ & ESE & SE & SSE & $S$ & SSW & SW & WSW & $\mathbf{W}$ & WNW & NW & NNW & Total \\
\hline 0-1mi & 0 & 0 & 0 & 0 & 0 & 0 & 0 & 0 & 0 & 0 & 0 & 0 & 0 & 0 & 0 & 0 & $\mathbf{0}$ \\
\hline $1-2$ & 0 & 0 & 0 & 0 & 0 & 0 & 0 & 0 & 0 & 0 & 0 & 0 & 0 & 0 & 0 & 0 & $\mathbf{0}$ \\
\hline $2-3$ & 0 & 0 & 0 & 0 & 0 & 0 & 0 & 0 & 0 & 0 & 0 & 0 & 0 & 0 & 0 & 0 & $\mathbf{0}$ \\
\hline $3-4$ & 0 & 0 & 2 & 4 & 4 & 4 & 0 & 0 & 0 & 0 & 0 & 0 & 0 & 0 & 0 & 0 & 14 \\
\hline $4-5$ & 0 & 0 & 1 & 5 & 5 & 6 & 1 & 0 & 0 & 0 & 0 & 0 & 0 & 0 & 0 & 0 & 18 \\
\hline $5-6$ & 0 & 0 & 1 & 3 & 6 & 7 & 1 & 0 & 0 & 0 & 0 & 0 & 0 & 0 & 0 & 0 & 18 \\
\hline 6-7 & 0 & 0 & 5 & 6 & 7 & 8 & 1 & 0 & 0 & 0 & 0 & 0 & 0 & 0 & 0 & 0 & 27 \\
\hline $7-8$ & 0 & 1 & 7 & 12 & 15 & 13 & 1 & 0 & 0 & 0 & 0 & 0 & 0 & 0 & 0 & 0 & 49 \\
\hline $8-9$ & 0 & 1 & 7 & 14 & 22 & 18 & 1 & 0 & 0 & 0 & 0 & 0 & 0 & 0 & 0 & 0 & 63 \\
\hline 9-10 & 0 & 1 & 10 & 15 & 53 & 19 & 1 & 0 & 0 & 0 & 0 & 0 & 0 & 0 & 0 & 4 & 103 \\
\hline 10-12 & 1 & 33 & 49 & 37 & 131 & 53 & 31 & 0 & 0 & 0 & 0 & 0 & 0 & 4 & 18 & 27 & 384 \\
\hline $12-14$ & 3 & 186 & 149 & 103 & 132 & 465 & 103 & 4 & 0 & 0 & 0 & 0 & 7 & 51 & 108 & 21 & 1,332 \\
\hline 14-16 & 38 & 61 & 813 & 400 & 300 & 764 & 202 & 0 & 0 & 0 & 0 & 0 & 14 & 90 & 88 & 41 & 2,811 \\
\hline 16-18 & 282 & 43 & 3,065 & 228 & 59 & 251 & 249 & 10 & 28 & 0 & 0 & 10 & 27 & 96 & 29 & 149 & 4,526 \\
\hline $18-20$ & 326 & 82 & 6,135 & 189 & 133 & 220 & 367 & 69 & 357 & 5 & 2 & 28 & 48 & 144 & 52 & 439 & 8,596 \\
\hline 20-25 & 397 & 274 & 632 & 289 & 621 & 328 & 1,039 & 18,225 & 7,106 & 286 & 16 & 34 & 1,183 & 3,535 & 345 & 2,188 & 36,498 \\
\hline $25-30$ & 21 & 575 & 1,725 & 98 & 3,095 & 96 & 302 & 32,968 & 5,231 & 1,578 & 415 & 32 & 0 & 554 & 441 & 159 & 47,290 \\
\hline 30-35 & 2,388 & 11,356 & 1,644 & 155 & 86 & 42 & 1,795 & 89,499 & 368 & 8,088 & 25,465 & 626 & 0 & 0 & 400 & 275 & 142,187 \\
\hline $35-40$ & 8,985 & 8,572 & 101 & 102 & 55 & 102 & 3,810 & 11,927 & 104 & 686 & 11,389 & 3,083 & 54 & 0 & 206 & 1,604 & 50,780 \\
\hline $40-50$ & 9,051 & 711 & 238 & 757 & 389 & 1,029 & 355 & 636 & 2,881 & 99 & 717 & 30,596 & 30,893 & 885 & 177 & 8,096 & 87,510 \\
\hline Total & 21,492 & 21,896 & 14,584 & 2,417 & 5,113 & 3,425 & 8,259 & 153,338 & 16,075 & 10,742 & 38,004 & 34,409 & 32,226 & 5,359 & 1,864 & 13,003 & 382,206 \\
\hline
\end{tabular}


Table 3.3. Distribution of Native American Population within 80 km (50 mi) of the Hanford 100 F Area Meteorological Station

\begin{tabular}{|c|c|c|c|c|c|c|c|c|c|c|c|c|c|c|c|c|c|}
\hline & \multicolumn{17}{|c|}{ Direction } \\
\hline $\begin{array}{l}\text { Distance } \\
\text { from } \\
\text { Reference } \\
\text { Point } \\
\text { (Miles) } \\
\end{array}$ & $\mathbf{N}$ & NNE & NE & ENE & $\mathbf{E}$ & ESE & SE & SSE & $\mathrm{S}$ & SSW & SW & WSW & $\mathbf{W}$ & WNW & NW & NNW & Total \\
\hline 0-1mi & 0 & 0 & 0 & 0 & 0 & 0 & 0 & 0 & 0 & 0 & 0 & 0 & 0 & 0 & 0 & 0 & $\mathbf{0}$ \\
\hline 1-2 & 0 & 0 & 0 & 0 & 0 & 0 & 0 & 0 & 0 & 0 & 0 & 0 & 0 & 0 & 0 & 0 & $\mathbf{0}$ \\
\hline $2-3$ & 0 & 0 & 0 & 0 & 0 & 0 & 0 & 0 & 0 & 0 & 0 & 0 & 0 & 0 & 0 & 0 & $\mathbf{0}$ \\
\hline $3-4$ & 0 & 0 & 0 & 0 & 0 & 0 & 0 & 0 & 0 & 0 & 0 & 0 & 0 & 0 & 0 & 0 & $\mathbf{0}$ \\
\hline 4-5 & 0 & 0 & 0 & 0 & 0 & 0 & 0 & 0 & 0 & 0 & 0 & 0 & 0 & 0 & 0 & 0 & $\mathbf{0}$ \\
\hline $5-6$ & 0 & 0 & 0 & 0 & 0 & 0 & 0 & 0 & 0 & 0 & 0 & 0 & 0 & 0 & 0 & 0 & $\mathbf{0}$ \\
\hline 6-7 & 0 & 0 & 0 & 0 & 0 & 0 & 0 & 0 & 0 & 0 & 0 & 0 & 0 & 0 & 0 & 0 & $\mathbf{0}$ \\
\hline $7-8$ & 0 & 0 & 0 & 0 & 0 & 0 & 0 & 0 & 0 & 0 & 0 & 0 & 0 & 0 & 0 & 0 & $\mathbf{0}$ \\
\hline $8-9$ & 0 & 0 & 0 & 0 & 0 & 0 & 0 & 0 & 0 & 0 & 0 & 0 & 0 & 0 & 0 & 0 & $\mathbf{0}$ \\
\hline 9-10 & 0 & 0 & 0 & 0 & 0 & 0 & 0 & 0 & 0 & 0 & 0 & 0 & 0 & 0 & 0 & 0 & $\mathbf{0}$ \\
\hline $10-12$ & 0 & 2 & 0 & 0 & 0 & 0 & 0 & 0 & 0 & 0 & 0 & 0 & 0 & 0 & 0 & 0 & 2 \\
\hline $12-14$ & 0 & 3 & 2 & 0 & 0 & 1 & 0 & 0 & 0 & 0 & 0 & 0 & 0 & 0 & 0 & 0 & 6 \\
\hline 14-16 & 0 & 0 & 5 & 1 & 2 & 3 & 1 & 0 & 0 & 0 & 0 & 0 & 0 & 0 & 0 & 0 & 12 \\
\hline $16-18$ & 0 & 0 & 14 & 1 & 0 & 0 & 2 & 0 & 0 & 0 & 0 & 0 & 0 & 5 & 0 & 0 & 22 \\
\hline $18-20$ & 3 & 0 & 60 & 0 & 0 & 0 & 5 & 1 & 2 & 0 & 0 & 0 & 0 & 0 & 1 & 2 & 74 \\
\hline $20-25$ & 1 & 2 & 1 & 0 & 4 & 1 & 3 & 142 & 53 & 6 & 0 & 0 & 2 & 22 & 0 & 11 & 248 \\
\hline $25-30$ & 0 & 0 & 13 & 0 & 33 & 1 & 0 & 244 & 45 & 10 & 1 & 0 & 0 & 4 & 3 & 0 & 354 \\
\hline 30-35 & 19 & 77 & 14 & 1 & 0 & 0 & 18 & 737 & 4 & 52 & 208 & 1 & 0 & 0 & 1 & 0 & 1,132 \\
\hline $35-40$ & 141 & 118 & 0 & 0 & 0 & 0 & 33 & 119 & 0 & 10 & 91 & 56 & 4 & 0 & 0 & 4 & 576 \\
\hline $40-50$ & 67 & 5 & 0 & 3 & 2 & 12 & 5 & 3 & 34 & 6 & 193 & 4,100 & 589 & 10 & 0 & 90 & 5,119 \\
\hline Total & 231 & 207 & 109 & 6 & 41 & 18 & 67 & 1,246 & 138 & 84 & 493 & 4,157 & 595 & 41 & 5 & 107 & 7,545 \\
\hline
\end{tabular}


Table 3.4. Distribution of Hispanic and Latino Population within 80 km (50 mi) of the Hanford 100 F Area Meteorological Station

\begin{tabular}{|c|c|c|c|c|c|c|c|c|c|c|c|c|c|c|c|c|c|}
\hline & \multicolumn{17}{|c|}{ Direction } \\
\hline $\begin{array}{l}\text { Distance } \\
\text { from } \\
\text { Reference } \\
\text { Point } \\
\text { (Miles) }\end{array}$ & $\mathbf{N}$ & NNE & NE & ENE & $\mathbf{E}$ & ESE & SE & SSE & $\mathbf{S}$ & SSW & SW & WSW & $\mathbf{W}$ & WNW & NW & NNW & Total \\
\hline 0-1mi & 0 & 0 & 0 & 0 & 0 & 0 & 0 & 0 & 0 & 0 & 0 & 0 & 0 & 0 & 0 & 0 & $\mathbf{0}$ \\
\hline $1-2$ & 0 & 0 & 0 & 0 & 0 & 0 & 0 & 0 & 0 & 0 & 0 & 0 & 0 & 0 & 0 & 0 & 0 \\
\hline $2-3$ & 0 & 0 & 0 & 0 & 0 & 0 & 0 & 0 & 0 & 0 & 0 & 0 & 0 & 0 & 0 & 0 & 0 \\
\hline $3-4$ & 0 & 0 & 0 & 0 & 0 & 0 & 0 & 0 & 0 & 0 & 0 & 0 & 0 & 0 & 0 & 0 & $\mathbf{0}$ \\
\hline $4-5$ & 0 & 0 & 0 & 1 & 1 & 1 & 0 & 0 & 0 & 0 & 0 & 0 & 0 & 0 & 0 & 0 & 3 \\
\hline $5-6$ & 0 & 0 & 0 & 1 & 1 & 1 & 0 & 0 & 0 & 0 & 0 & 0 & 0 & 0 & 0 & 0 & 3 \\
\hline 6-7 & 0 & 0 & 0 & 2 & 1 & 1 & 0 & 0 & 0 & 0 & 0 & 0 & 0 & 0 & 0 & 0 & 4 \\
\hline $7-8$ & 0 & 0 & 0 & 5 & 4 & 1 & 0 & 0 & 0 & 0 & 0 & 0 & 0 & 0 & 0 & 0 & 10 \\
\hline $8-9$ & 0 & 0 & 0 & 7 & 4 & 2 & 0 & 0 & 0 & 0 & 0 & 0 & 0 & 0 & 0 & 0 & 13 \\
\hline 9-10 & 0 & 1 & 3 & 6 & 8 & 3 & 0 & 0 & 0 & 0 & 0 & 0 & 0 & 0 & 0 & 3 & 24 \\
\hline 10-12 & 0 & 15 & 28 & 8 & 43 & 10 & 7 & 0 & 0 & 0 & 0 & 0 & 0 & 2 & 12 & 22 & 147 \\
\hline $12-14$ & 2 & 89 & 77 & 22 & 34 & 333 & 49 & 0 & 0 & 0 & 0 & 0 & 4 & 30 & 74 & 7 & 721 \\
\hline 14-16 & 17 & 29 & 297 & 148 & 156 & 523 & 85 & 0 & 0 & 0 & 0 & 0 & 9 & 64 & 58 & 12 & 1,398 \\
\hline 16-18 & 162 & 12 & 2,400 & 79 & 12 & 87 & 128 & 5 & 1 & 0 & 0 & 4 & 11 & 49 & 13 & 88 & 3,051 \\
\hline $18-20$ & 147 & 29 & 3,935 & 43 & 28 & 77 & 165 & 18 & 13 & 1 & 0 & 9 & 7 & 73 & 17 & 307 & 4,869 \\
\hline 20-25 & 113 & 70 & 358 & 56 & 299 & 77 & 294 & 887 & 596 & 85 & 5 & 3 & 574 & 2,950 & 186 & 1,520 & 8,073 \\
\hline $25-30$ & 0 & 40 & 1,076 & 36 & 1,247 & 19 & 54 & 2,346 & 613 & 663 & 195 & 3 & 0 & 404 & 239 & 65 & 7,000 \\
\hline $30-35$ & 189 & 2,276 & 948 & 31 & 11 & 4 & 1,145 & 25,420 & 22 & 2,568 & 17,287 & 259 & 0 & 0 & 154 & 24 & 50,338 \\
\hline $35-40$ & 1,913 & 2,347 & 3 & 24 & 1 & 24 & 459 & 1,019 & 12 & 130 & 6,860 & 1,285 & 8 & 0 & 61 & 608 & 14,754 \\
\hline $40-50$ & 891 & 106 & 15 & 71 & 35 & 830 & 64 & 106 & 811 & 33 & 248 & 17,757 & 11,625 & 39 & 11 & 4,145 & 36,787 \\
\hline Total & 3,434 & 5,014 & 9,140 & 540 & 1,885 & 1,993 & 2,450 & 29,801 & 2,068 & 3,480 & 24,595 & 19,320 & 12,238 & 3,611 & 825 & 6,801 & 127,195 \\
\hline
\end{tabular}


Table 3.5. Distribution of Total Minority Population within 80 km (50 mi) of the Hanford 100 F Area Meteorological Station

\begin{tabular}{|c|c|c|c|c|c|c|c|c|c|c|c|c|c|c|c|c|c|}
\hline & \multicolumn{17}{|c|}{ Direction } \\
\hline $\begin{array}{l}\text { Distance } \\
\text { from } \\
\text { Reference } \\
\text { Point } \\
\text { (Miles) } \\
\end{array}$ & $\mathbf{N}$ & NNE & $\mathrm{NE}$ & ENE & $\mathbf{E}$ & ESE & SE & SSE & $\mathbf{S}$ & SSW & SW & WSW & W & WNW & NW & NNW & Total \\
\hline 0-1mi & 0 & 0 & 0 & 0 & 0 & 0 & 0 & 0 & 0 & 0 & 0 & 0 & 0 & 0 & 0 & 0 & $\mathbf{0}$ \\
\hline $1-2$ & 0 & 0 & 0 & 0 & 0 & 0 & 0 & 0 & 0 & 0 & 0 & 0 & 0 & 0 & 0 & 0 & $\mathbf{0}$ \\
\hline $2-3$ & 0 & 0 & 0 & 0 & 0 & 0 & 0 & 0 & 0 & 0 & 0 & 0 & 0 & 0 & 0 & 0 & $\mathbf{0}$ \\
\hline $3-4$ & 0 & 0 & 0 & 1 & 1 & 1 & 0 & 0 & 0 & 0 & 0 & 0 & 0 & 0 & 0 & 0 & 3 \\
\hline $4-5$ & 0 & 0 & 0 & 1 & 1 & 1 & 0 & 0 & 0 & 0 & 0 & 0 & 0 & 0 & 0 & 0 & 3 \\
\hline $5-6$ & 0 & 0 & 0 & 1 & 1 & 1 & 0 & 0 & 0 & 0 & 0 & 0 & 0 & 0 & 0 & 0 & 3 \\
\hline $6-7$ & 0 & 0 & 0 & 2 & 2 & 1 & 0 & 0 & 0 & 0 & 0 & 0 & 0 & 0 & 0 & 0 & 5 \\
\hline $7-8$ & 0 & 0 & 0 & 5 & 4 & 1 & 0 & 0 & 0 & 0 & 0 & 0 & 0 & 0 & 0 & 0 & 10 \\
\hline $8-9$ & 0 & 0 & 0 & 7 & 4 & 2 & 0 & 0 & 0 & 0 & 0 & 0 & 0 & 0 & 0 & 0 & 13 \\
\hline 9-10 & 0 & 1 & 3 & 6 & 8 & 3 & 0 & 0 & 0 & 0 & 0 & 0 & 0 & 0 & 0 & 3 & 24 \\
\hline $10-12$ & 0 & 21 & 29 & 8 & 46 & 11 & 9 & 0 & 0 & 0 & 0 & 0 & 0 & 3 & 12 & 22 & 161 \\
\hline $12-14$ & 2 & 98 & 78 & 22 & 38 & 338 & 50 & 2 & 0 & 0 & 0 & 0 & 4 & 32 & 74 & 7 & 745 \\
\hline $14-16$ & 18 & 29 & 311 & 150 & 163 & 533 & 87 & 0 & 0 & 0 & 0 & 0 & 9 & 66 & 58 & 16 & 1,440 \\
\hline 16-18 & 163 & 12 & 2,435 & 82 & 14 & 94 & 132 & 5 & 1 & 0 & 0 & 4 & 14 & 55 & 14 & 91 & 3,116 \\
\hline $18-20$ & 150 & 34 & 4,049 & 51 & 33 & 79 & 171 & 20 & 20 & 1 & 0 & 9 & 15 & 73 & 19 & 315 & 5,039 \\
\hline 20-25 & 122 & 76 & 371 & 65 & 310 & 82 & 317 & 2,459 & 851 & 87 & 5 & 4 & 600 & 3,005 & 188 & 1,557 & 10,099 \\
\hline $25-30$ & 0 & 59 & 1,105 & 38 & 1,557 & 19 & 61 & 4,488 & 846 & 692 & 214 & 6 & 0 & 408 & 246 & 70 & 9,809 \\
\hline 30-35 & 273 & 2,861 & 983 & 41 & 14 & 4 & 1,228 & 30,961 & 36 & 2,755 & 17,861 & 273 & 0 & 0 & 168 & 32 & 57,490 \\
\hline $35-40$ & 2,707 & 2,830 & 5 & 29 & 1 & 35 & 595 & 1,452 & 16 & 144 & 7,113 & 1,415 & 14 & 0 & 65 & 648 & 17,069 \\
\hline $40-50$ & 1,238 & 136 & 21 & 88 & 45 & 849 & 72 & 121 & 964 & 39 & 473 & 22,529 & 13,449 & 68 & 11 & 4,347 & 44,450 \\
\hline Total & 4,673 & 6,157 & 9,390 & 597 & 2,242 & 2,054 & 2,722 & 39,508 & 2,734 & 3,718 & 25,666 & 24,240 & 14,105 & 3,710 & 855 & 7,108 & 149,479 \\
\hline
\end{tabular}


Table 3.6. Distribution of Low-Income Population within $80 \mathrm{~km}$ (50 mi) of the Hanford 100 F Area Meteorological Station

\begin{tabular}{|c|c|c|c|c|c|c|c|c|c|c|c|c|c|c|c|c|c|}
\hline & \multicolumn{17}{|c|}{ Direction } \\
\hline $\begin{array}{l}\text { Distance } \\
\text { from } \\
\text { Reference } \\
\text { Point } \\
\text { (Miles) } \\
\end{array}$ & $\mathbf{N}$ & NNE & NE & ENE & $\mathbf{E}$ & ESE & SE & SSE & $S$ & SSW & SW & WSW & $\mathbf{W}$ & WNW & NW & NNW & Total \\
\hline 0-1mi & 0 & 0 & 0 & 0 & 0 & 0 & 0 & 0 & 0 & 0 & 0 & 0 & 0 & 0 & 0 & 0 & $\mathbf{0}$ \\
\hline $1-2$ & 0 & 0 & 0 & 0 & 0 & 0 & 0 & 0 & 0 & 0 & 0 & 0 & 0 & 0 & 0 & 0 & 0 \\
\hline $2-3$ & 0 & 0 & 0 & 0 & 0 & 0 & 0 & 0 & 0 & 0 & 0 & 0 & 0 & 0 & 0 & 0 & 0 \\
\hline $3-4$ & 0 & 0 & 0 & 0 & 0 & 0 & 0 & 0 & 0 & 0 & 0 & 0 & 0 & 0 & 0 & 0 & $\mathbf{0}$ \\
\hline $4-5$ & 0 & 0 & 0 & 0 & 0 & 0 & 0 & 0 & 0 & 0 & 0 & 0 & 0 & 0 & 0 & 0 & 0 \\
\hline $5-6$ & 0 & 0 & 0 & 0 & 0 & 0 & 0 & 0 & 0 & 0 & 0 & 0 & 0 & 0 & 0 & 0 & 0 \\
\hline 6-7 & 0 & 0 & 0 & 0 & 0 & 1 & 0 & 0 & 0 & 0 & 0 & 0 & 0 & 0 & 0 & 0 & 1 \\
\hline $7-8$ & 0 & 0 & 0 & 1 & 1 & 1 & 0 & 0 & 0 & 0 & 0 & 0 & 0 & 0 & 0 & 0 & 3 \\
\hline $8-9$ & 0 & 0 & 0 & 1 & 1 & 1 & 0 & 0 & 0 & 0 & 0 & 0 & 0 & 0 & 0 & 0 & 3 \\
\hline 9-10 & 0 & 0 & 1 & 1 & 3 & 1 & 0 & 0 & 0 & 0 & 0 & 0 & 0 & 0 & 0 & 1 & 7 \\
\hline 10-12 & 0 & 2 & 3 & 2 & 9 & 4 & 4 & 0 & 0 & 0 & 0 & 0 & 0 & 1 & 4 & 6 & 35 \\
\hline $12-14$ & 0 & 13 & 10 & 9 & 24 & 118 & 12 & 0 & 0 & 0 & 0 & 0 & 2 & 11 & 23 & 5 & 227 \\
\hline 14-16 & 5 & 11 & 111 & 67 & 88 & 211 & 24 & 0 & 0 & 0 & 0 & 0 & 3 & 19 & 19 & 8 & 566 \\
\hline 16-18 & 36 & 9 & 575 & 39 & 17 & 46 & 30 & 1 & 1 & 0 & 0 & 1 & 6 & 21 & 6 & 20 & 808 \\
\hline $18-20$ & 42 & 18 & 1,494 & 32 & 39 & 30 & 44 & 8 & 15 & 0 & 0 & 3 & 10 & 31 & 8 & 88 & 1,862 \\
\hline 20-25 & 51 & 36 & 70 & 25 & 141 & 19 & 102 & 1,495 & 473 & 23 & 2 & 4 & 257 & 769 & 101 & 514 & 4,082 \\
\hline 25-30 & 2 & 46 & 329 & 6 & 575 & 9 & 24 & 2,181 & 579 & 230 & 62 & 5 & 0 & 154 & 151 & 41 & 4,394 \\
\hline 30-35 & 277 & 1,491 & 306 & 19 & 11 & 5 & 376 & 14,426 & 12 & 1,232 & 7,236 & 99 & 0 & 0 & 98 & 66 & 25,654 \\
\hline $35-40$ & 2,169 & 1,190 & 11 & 13 & 8 & 11 & 263 & 673 & 15 & 95 & 2,596 & 831 & 6 & 0 & 54 & 295 & 8,230 \\
\hline $40-50$ & 1,146 & 71 & 35 & 77 & 61 & 209 & 43 & 33 & 646 & 19 & 176 & 8,587 & 7,240 & 101 & 41 & 1,444 & 19,929 \\
\hline Total & 3,728 & 2,887 & 2,945 & 292 & 978 & 666 & 922 & 18,817 & 1,741 & 1,599 & 10,072 & 9,530 & 7,524 & 1,107 & 505 & 2,488 & 65,801 \\
\hline
\end{tabular}




\section{3 $100 \mathrm{~K}$ Area}

The $100 \mathrm{~K}$ Area Meteorological Station is near the northwest corner of the Hanford Site. At the 2000 Census there were about 481,900 people residing within $80 \mathrm{~km}$ (50 mi) of this location. Because $100 \mathrm{~K}$ is located several miles west of $100 \mathrm{~F}$, the 50 -mile radius for this reference point includes the city of Yakima and several surrounding towns located west of the site; thus, the total population for this reference point is higher than the population for $100 \mathrm{~F}$. Based on census block data, about 176,800 (37\%) of these residents were minorities. The Hispanic and Latino population (all races) was the single largest minority group, with 148,100 members (31\% of the population). There were 10,100 Native Americans (2\% of the total). There were an estimated 80,600 low-income individuals (about $17 \%$ of the population).

Figures 3.8 to 3.13 and Tables 3.7 to 3.11 show population distributions for total, minority, and lowincome populations living within 50 miles of the $100 \mathrm{~K}$ Area Meteorological Station on the Hanford Site.

Although the $100 \mathrm{~K}$ Area Meteorological Station is comparatively isolated in the northwest corner of the Hanford site, about 400 people live in the farming areas toward the north, ranging from northeast to northwest. About 1,700 people live within 15 miles, and 12,400 people live within 20 miles, mostly from the WNW to ENE. There are about 16,000 people living between 20 and 25 miles from the $100 \mathrm{~K}$ Area Meteorological Station, mostly to the ENE. There are small concentrations of Native Americans living between 20 and 25 miles to the ENE, and a few hundred living in the Tri-Cities area 20-50 miles to the south and southeast. The largest Native American population is 40-50 miles WSW in the Yakima area, with over 4,000 individuals. Hispanic and Latino populations are located 16-18 miles to the north (over 1,500 individuals) and WNW (2,700 individuals), 20-25 miles to the ESE to ENE (7,500 individuals), 2530 miles SSE to SSW (3,800 individuals), 30-35 miles to the SSW (10,000 individuals) and SW (14,000 individuals), 35-40 miles to the SE (22,400 individuals) and NNE (4,900 individuals), and 40-50 miles to the WSW (11,200 individuals) and due west (30,200 individuals). 


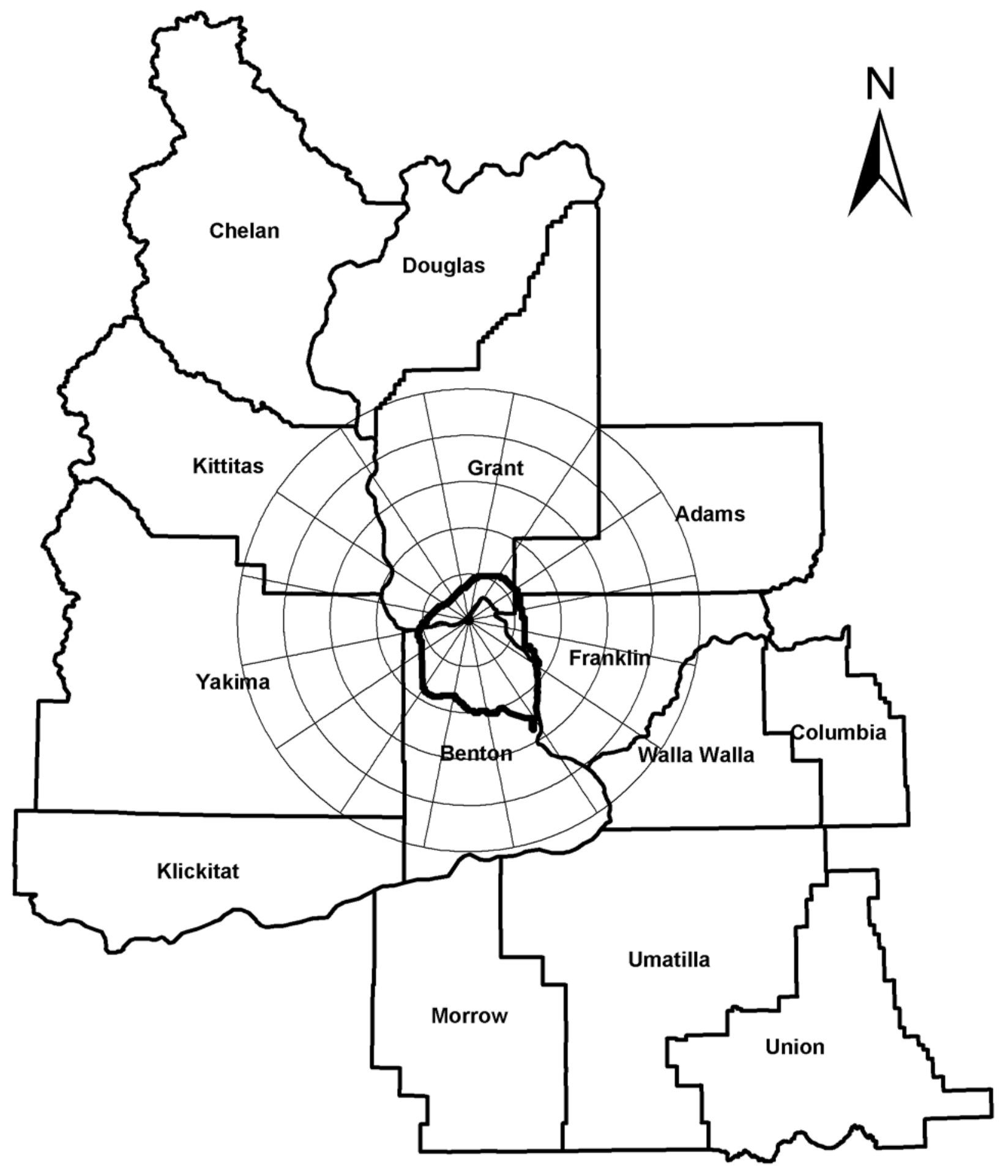

Figure 3.8. Map of 80-km (50-mi) Region Surrounding the $100 \mathrm{~K}$ Area Meteorological Station 


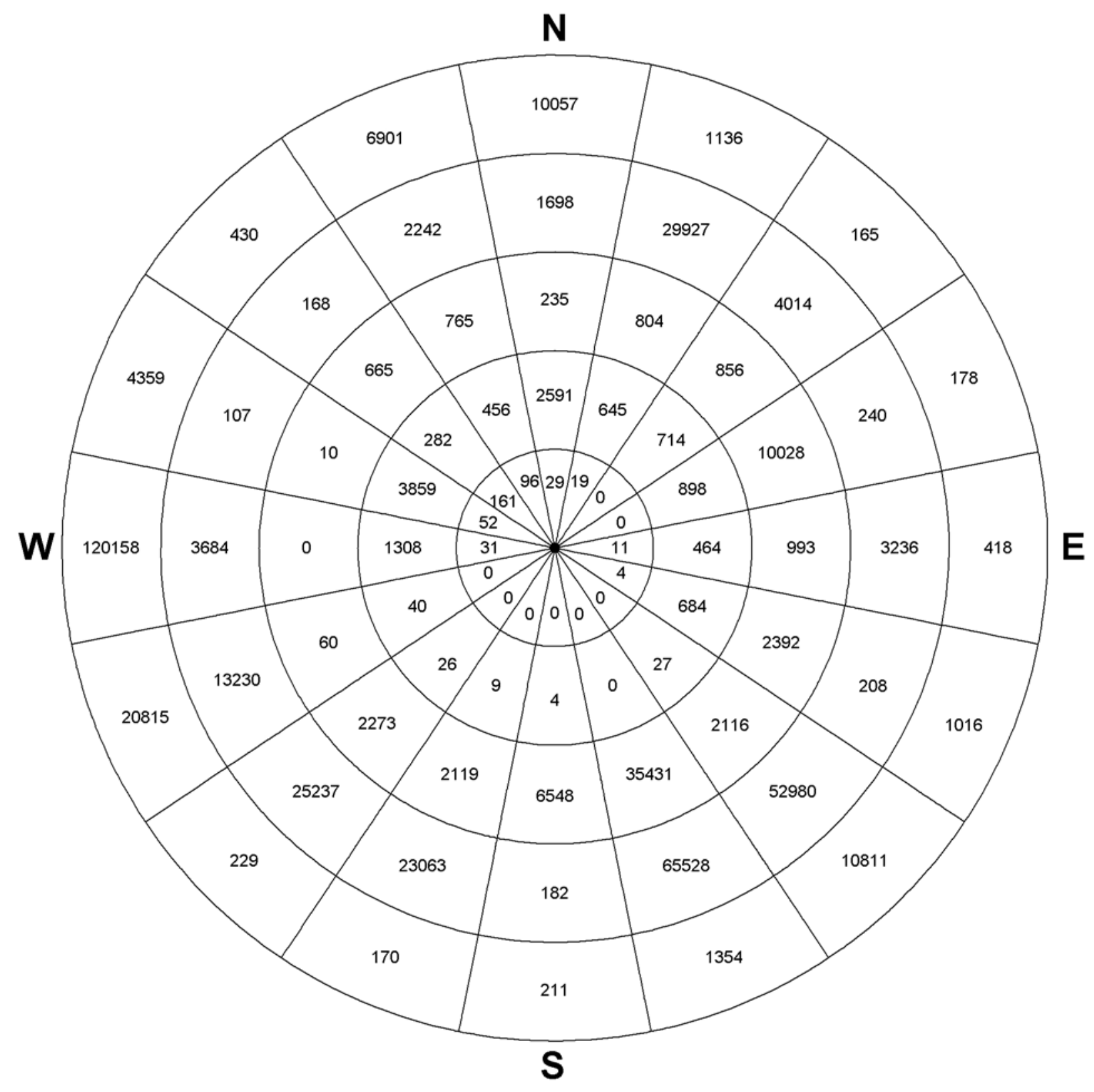

Figure 3.9. Map of Total Resident Population within $80 \mathrm{~km}$ (50 mi) of the Hanford $100 \mathrm{~K}$ Area Meteorological Station, in 16-km (10-mi) Increments by Distance and Direction 


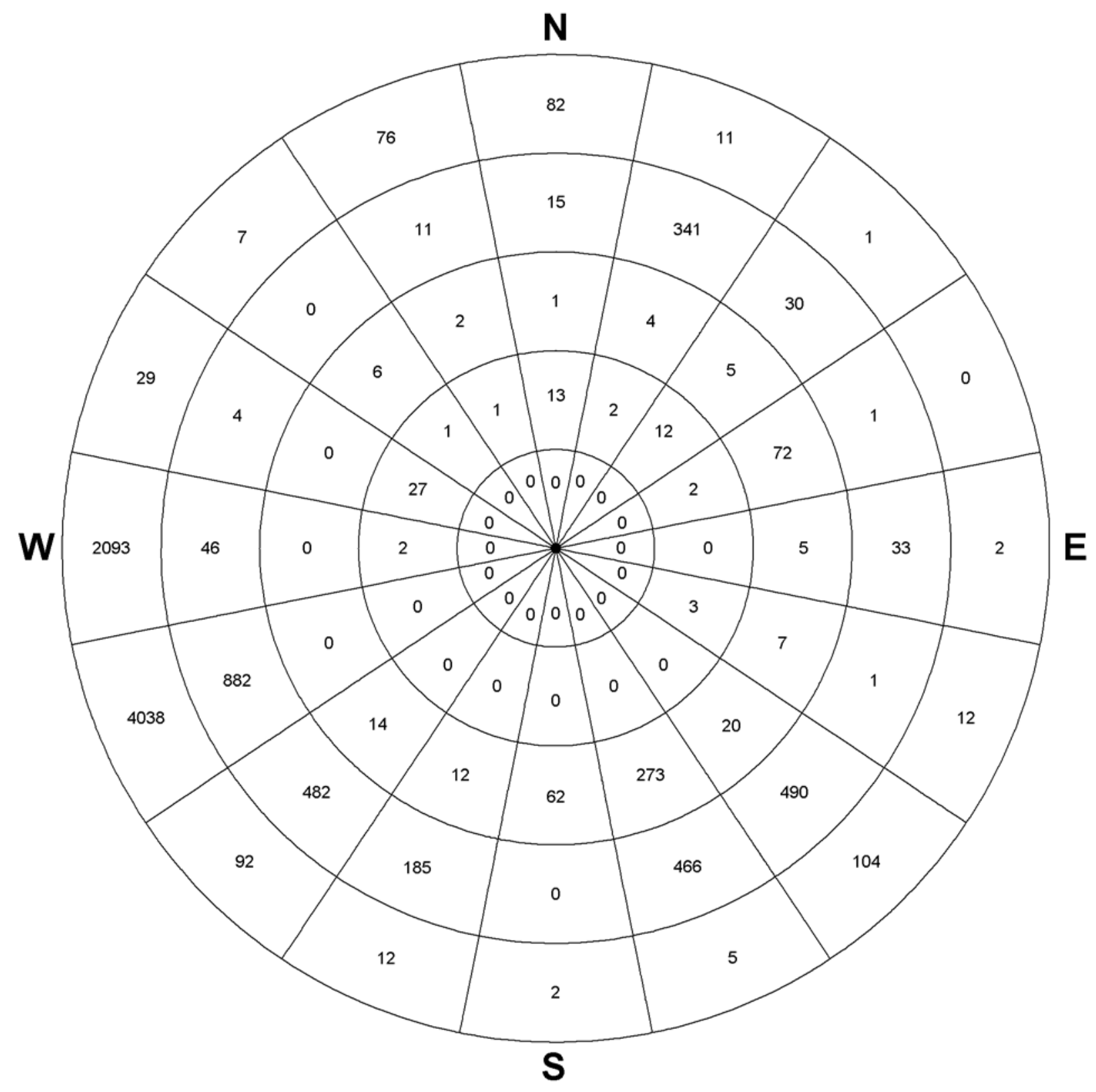

Figure 3.10. Map of Native American Population within $80 \mathrm{~km}(50 \mathrm{mi})$ of the Hanford $100 \mathrm{~K}$ Area Meteorological Station, in 16-km (10-mi) Increments by Distance and Direction 


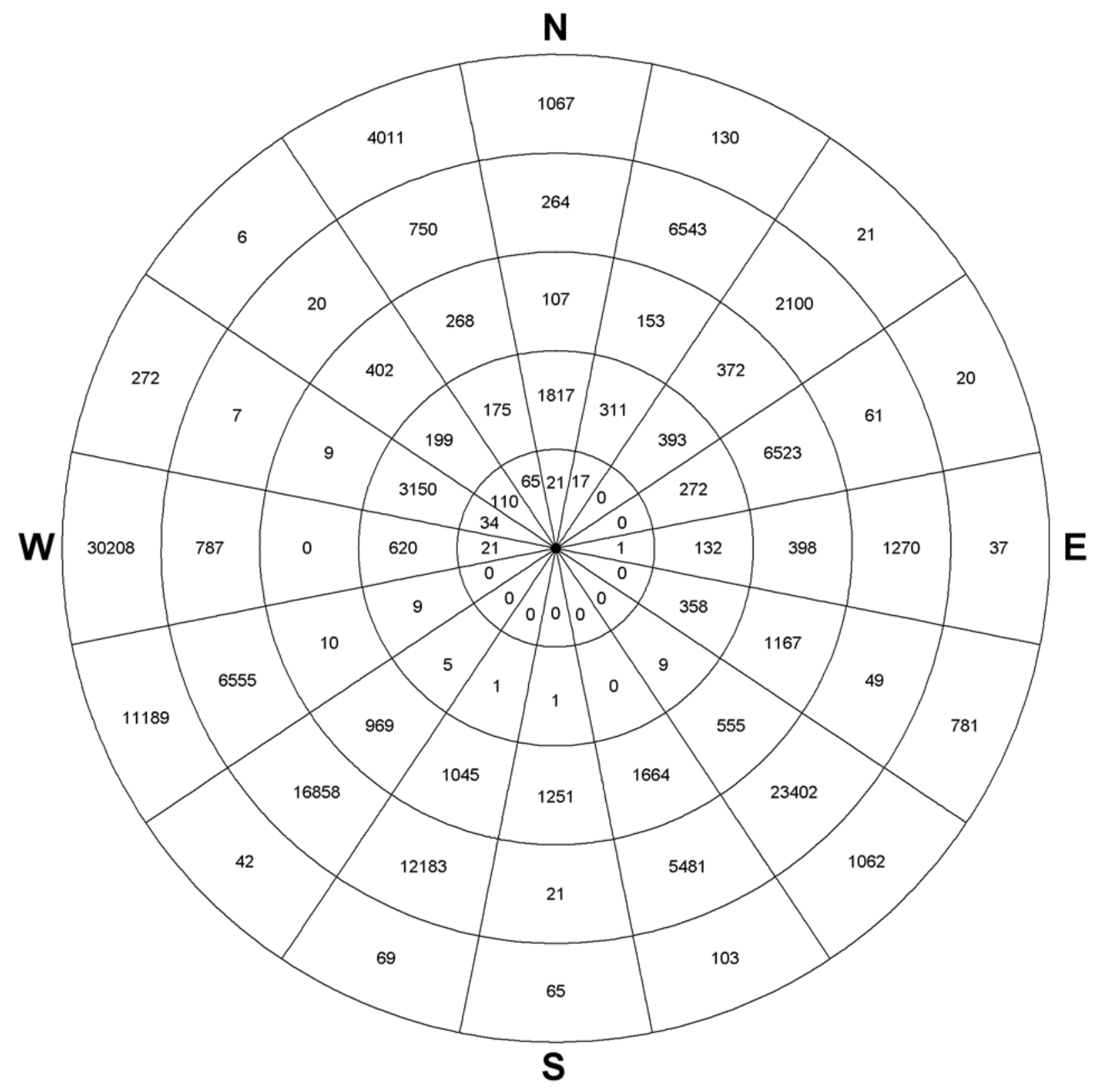

Figure 3.11. Map of Hispanic and Latino Population within $80 \mathrm{~km}(50 \mathrm{mi})$ of the Hanford $100 \mathrm{~K}$ Area Meteorological Station, in 16-km (10-mi) Increments by Distance and Direction 


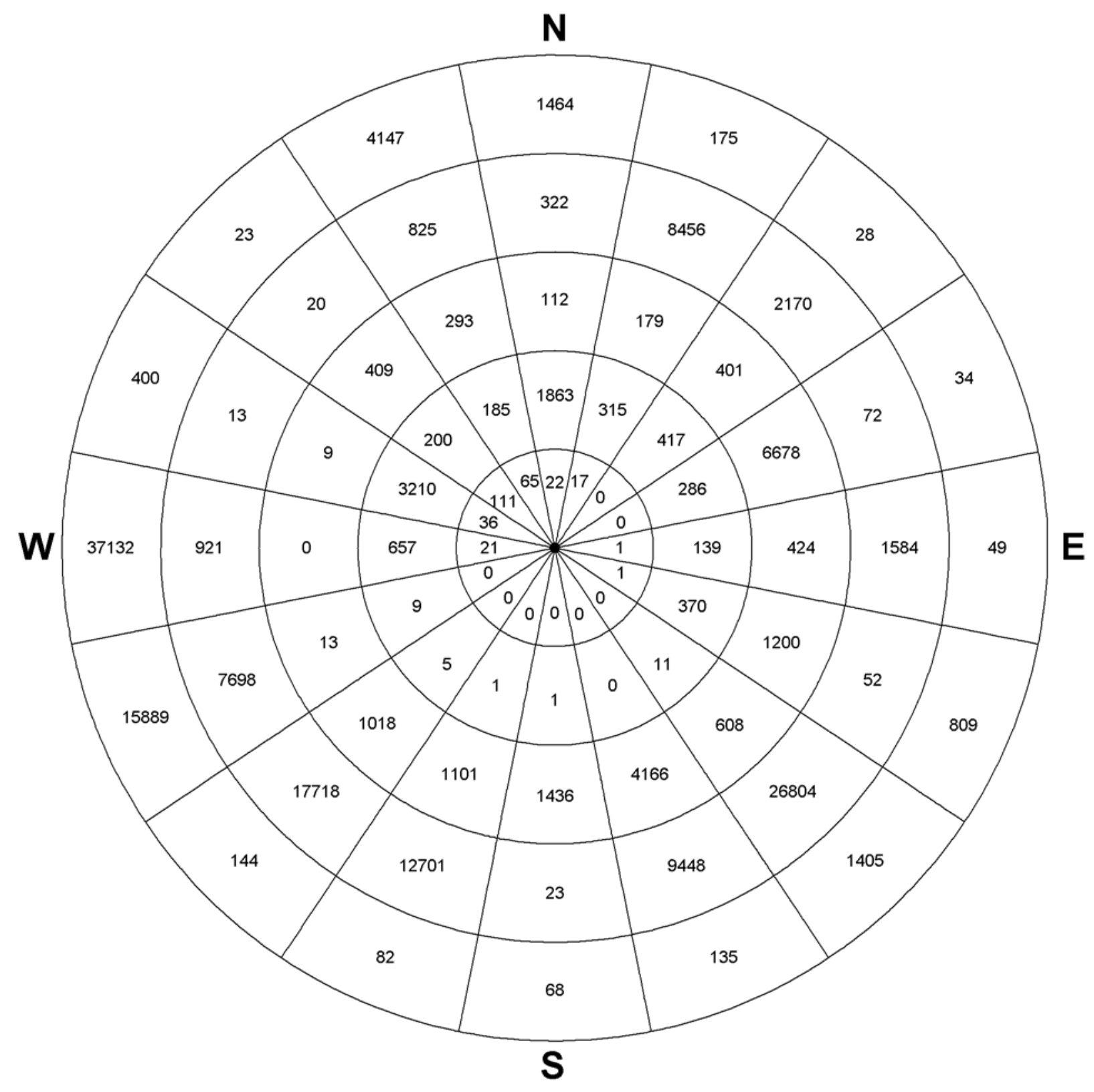

Figure 3.12. Map of Total Minority Population within $80 \mathrm{~km}(50 \mathrm{mi})$ of the Hanford $100 \mathrm{~K}$ Area Meteorological Station, in 16-km (10-mi) Increments by Distance and Direction 


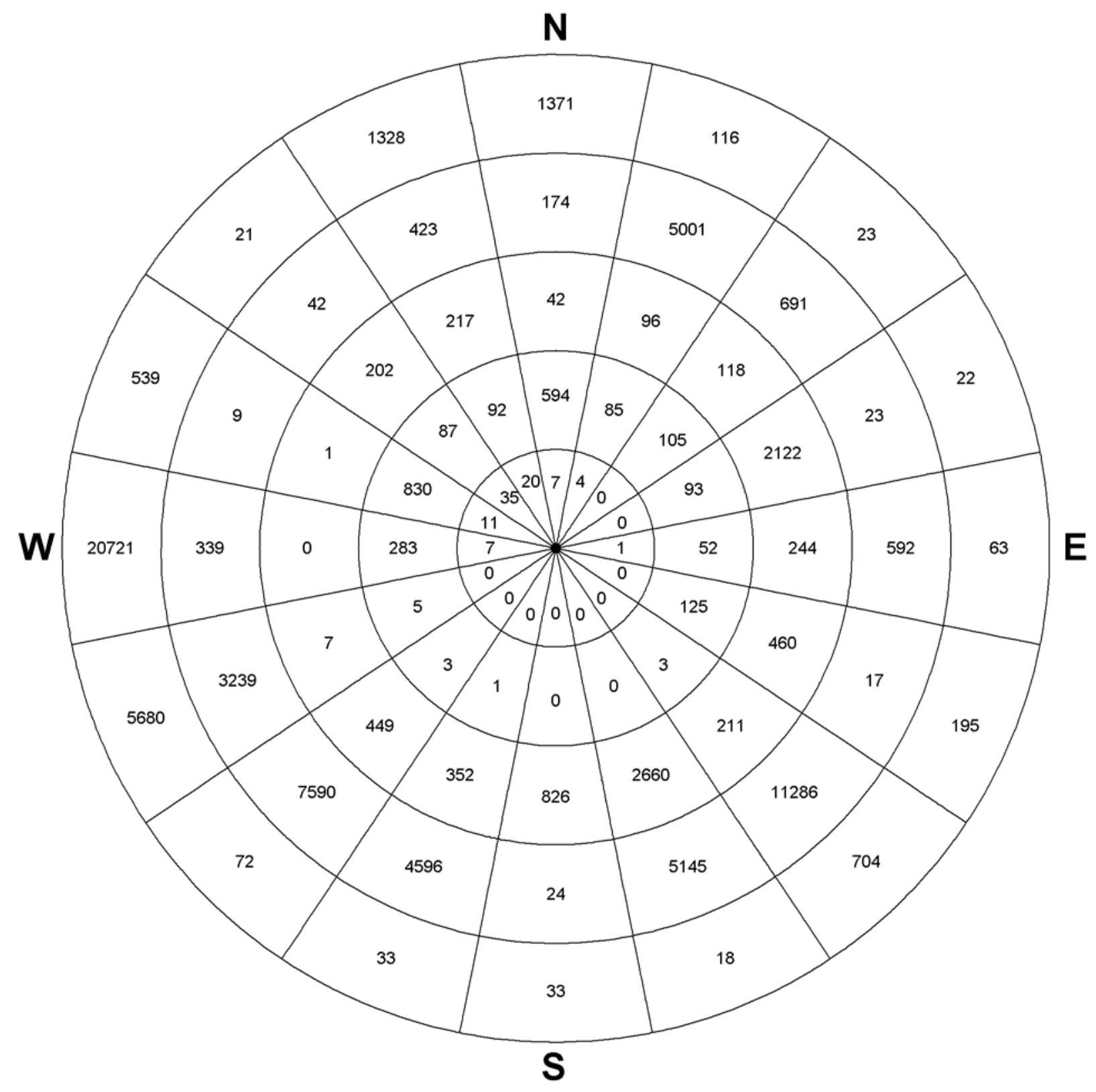

Figure 3.13. Map of Low-Income Population within $80 \mathrm{~km}$ (50 mi) of the Hanford $100 \mathrm{~K}$ Area Meteorological Station, in 16-km (10-mi) Increments by Distance and Direction 
Table 3.7. Distribution of Total Resident Population within 80 km (50 mi) of the Hanford $100 \mathrm{~K}$ Area Meteorological Station

\begin{tabular}{|c|c|c|c|c|c|c|c|c|c|c|c|c|c|c|c|c|c|}
\hline \multirow[b]{2}{*}{$\begin{array}{l}\text { Distance } \\
\text { from } \\
\text { Reference } \\
\text { Point } \\
\text { (Miles) }\end{array}$} & \multicolumn{17}{|c|}{ Direction } \\
\hline & $\mathbf{N}$ & NNE & NE & ENE & $\mathbf{E}$ & ESE & SE & SSE & $S$ & SSW & SW & WSW & $\mathbf{W}$ & WNW & NW & NNW & Total \\
\hline 0-1mi & 0 & 0 & 0 & 0 & 0 & 0 & 0 & 0 & 0 & 0 & 0 & 0 & 0 & 0 & 0 & 0 & 0 \\
\hline $1-2$ & 0 & 0 & 0 & 0 & 0 & 0 & 0 & 0 & 0 & 0 & 0 & 0 & 0 & 0 & 0 & 0 & $\mathbf{0}$ \\
\hline $2-3$ & 0 & 0 & 0 & 0 & 0 & 0 & 0 & 0 & 0 & 0 & 0 & 0 & 0 & 0 & 0 & 0 & $\mathbf{0}$ \\
\hline $3-4$ & 0 & 0 & 0 & 0 & 0 & 0 & 0 & 0 & 0 & 0 & 0 & 0 & 0 & 0 & 0 & 0 & $\mathbf{0}$ \\
\hline $4-5$ & 0 & 0 & 0 & 0 & 0 & 0 & 0 & 0 & 0 & 0 & 0 & 0 & 0 & 0 & 0 & 0 & 0 \\
\hline $5-6$ & 0 & 0 & 0 & 0 & 0 & 0 & 0 & 0 & 0 & 0 & 0 & 0 & 1 & 2 & 1 & 1 & 5 \\
\hline $6-7$ & 1 & 0 & 0 & 0 & 0 & 0 & 0 & 0 & 0 & 0 & 0 & 0 & 4 & 6 & 9 & 11 & 31 \\
\hline $7-8$ & 4 & 1 & 0 & 0 & 0 & 0 & 0 & 0 & 0 & 0 & 0 & 0 & 5 & 8 & 35 & 28 & 81 \\
\hline $8-9$ & 12 & 9 & 0 & 0 & 2 & 0 & 0 & 0 & 0 & 0 & 0 & 0 & 9 & 18 & 64 & 40 & 154 \\
\hline 9-10 & 12 & 9 & 0 & 0 & 9 & 4 & 0 & 0 & 0 & 0 & 0 & 0 & 12 & 18 & 52 & 16 & 132 \\
\hline $10-12$ & 16 & 5 & 0 & 11 & 19 & 15 & 0 & 0 & 0 & 0 & 0 & 1 & 34 & 72 & 52 & 14 & 239 \\
\hline $12-14$ & 51 & 17 & 16 & 25 & 39 & 24 & 0 & 0 & 0 & 0 & 5 & 12 & 57 & 134 & 17 & 33 & 430 \\
\hline 14-16 & 159 & 90 & 131 & 51 & 85 & 47 & 0 & 0 & 0 & 0 & 9 & 14 & 580 & 119 & 1 & 73 & 1,359 \\
\hline 16-18 & 2,079 & 214 & 220 & 161 & 152 & 95 & 4 & 0 & 1 & 1 & 10 & 9 & 636 & 3,098 & 54 & 143 & 6,877 \\
\hline 18-20 & 286 & 319 & 347 & 650 & 169 & 503 & 23 & 0 & 3 & 8 & 2 & 4 & 1 & 436 & 158 & 193 & 3,102 \\
\hline 20-25 & 190 & 560 & 387 & 9,641 & 278 & 1,707 & 547 & 935 & 882 & 36 & 49 & 15 & 0 & 10 & 523 & 348 & 16,108 \\
\hline $25-30$ & 45 & 244 & 469 & 387 & 715 & 685 & 1,569 & 34,496 & 5,666 & 2,083 & 2,224 & 45 & 0 & 0 & 142 & 417 & 49,187 \\
\hline $30-35$ & 502 & 9,646 & 3,355 & 86 & 3,135 & 147 & 5,806 & 27,272 & 88 & 20,072 & 20,998 & 1,097 & 43 & 0 & 39 & 1,365 & 93,651 \\
\hline $35-40$ & 1,196 & 20,281 & 659 & 154 & 101 & 61 & 47,174 & 38,256 & 94 & 2,991 & 4,239 & 12,133 & 3,641 & 107 & 129 & 877 & 132,093 \\
\hline $40-50$ & 10,057 & 1,136 & 165 & 178 & 418 & 1,016 & 10,811 & 1,354 & 211 & 170 & 229 & 20,815 & 120,158 & 4,359 & 430 & 6,901 & 178,408 \\
\hline Total & 14,610 & 32,531 & 5,749 & 11,344 & 5,122 & 4,304 & 65,934 & 102,313 & 6,945 & 25,361 & 27,765 & 34,145 & 125,181 & 8,387 & 1,706 & 10,460 & 481,857 \\
\hline
\end{tabular}


Table 3.8. Distribution of Native American Population within 80 km (50 mi) of the Hanford 100 K Area Meteorological Station

\begin{tabular}{|c|c|c|c|c|c|c|c|c|c|c|c|c|c|c|c|c|c|}
\hline & \multicolumn{17}{|c|}{ Direction } \\
\hline $\begin{array}{l}\text { Distance } \\
\text { from } \\
\text { Reference } \\
\text { Point } \\
\text { (Miles) } \\
\end{array}$ & $\mathbf{N}$ & NNE & $\mathrm{NE}$ & ENE & $\mathbf{E}$ & ESE & SE & SSE & $\mathbf{S}$ & SSW & SW & WSW & $\mathbf{W}$ & WNW & NW & NNW & Total \\
\hline 0-1mi & 0 & 0 & 0 & 0 & 0 & 0 & 0 & 0 & 0 & 0 & 0 & 0 & 0 & 0 & 0 & 0 & $\mathbf{0}$ \\
\hline 1-2 & 0 & 0 & 0 & 0 & 0 & 0 & 0 & 0 & 0 & 0 & 0 & 0 & 0 & 0 & 0 & 0 & 0 \\
\hline $2-3$ & 0 & 0 & 0 & 0 & 0 & 0 & 0 & 0 & 0 & 0 & 0 & 0 & 0 & 0 & 0 & 0 & 0 \\
\hline $3-4$ & 0 & 0 & 0 & 0 & 0 & 0 & 0 & 0 & 0 & 0 & 0 & 0 & 0 & 0 & 0 & 0 & 0 \\
\hline 4-5 & 0 & 0 & 0 & 0 & 0 & 0 & 0 & 0 & 0 & 0 & 0 & 0 & 0 & 0 & 0 & 0 & 0 \\
\hline 5-6 & 0 & 0 & 0 & 0 & 0 & 0 & 0 & 0 & 0 & 0 & 0 & 0 & 0 & 0 & 0 & 0 & 0 \\
\hline $6-7$ & 0 & 0 & 0 & 0 & 0 & 0 & 0 & 0 & 0 & 0 & 0 & 0 & 0 & 0 & 0 & 0 & $\mathbf{0}$ \\
\hline $7-8$ & 0 & 0 & 0 & 0 & 0 & 0 & 0 & 0 & 0 & 0 & 0 & 0 & 0 & 0 & 0 & 0 & 0 \\
\hline $8-9$ & 0 & 0 & 0 & 0 & 0 & 0 & 0 & 0 & 0 & 0 & 0 & 0 & 0 & 0 & 0 & 0 & 0 \\
\hline 9-10 & 0 & 0 & 0 & 0 & 0 & 0 & 0 & 0 & 0 & 0 & 0 & 0 & 0 & 0 & 0 & 0 & 0 \\
\hline 10-12 & 0 & 0 & 0 & 0 & 0 & 0 & 0 & 0 & 0 & 0 & 0 & 0 & 0 & 5 & 0 & 0 & 5 \\
\hline $12-14$ & 0 & 0 & 1 & 0 & 0 & 0 & 0 & 0 & 0 & 0 & 0 & 0 & 0 & 0 & 0 & 0 & 1 \\
\hline 14-16 & 0 & 0 & 3 & 0 & 0 & 0 & 0 & 0 & 0 & 0 & 0 & 0 & 2 & 0 & 0 & 1 & 6 \\
\hline $16-18$ & 10 & 1 & 1 & 1 & 0 & 1 & 0 & 0 & 0 & 0 & 0 & 0 & 0 & 20 & 0 & 0 & 34 \\
\hline $18-20$ & 3 & 1 & 7 & 1 & 0 & 2 & 0 & 0 & 0 & 0 & 0 & 0 & 0 & 2 & 1 & 0 & 17 \\
\hline 20-25 & 0 & 4 & 3 & 72 & 2 & 5 & 8 & 4 & 11 & 0 & 0 & 0 & 0 & 0 & 5 & 1 & 115 \\
\hline 25-30 & 1 & 0 & 2 & 0 & 3 & 2 & 12 & 269 & 51 & 12 & 14 & 0 & 0 & 0 & 1 & 1 & 368 \\
\hline $30-35$ & 1 & 66 & 29 & 0 & 33 & 1 & 34 & 174 & 0 & 164 & 164 & 21 & 5 & 0 & 0 & 4 & 696 \\
\hline $35-40$ & 14 & 275 & 1 & 1 & 0 & 0 & 456 & 292 & 0 & 21 & 318 & 861 & 41 & 4 & 0 & 7 & 2,291 \\
\hline $40-50$ & 82 & 11 & 1 & 0 & 2 & 12 & 104 & 5 & 2 & 12 & 92 & 4,038 & 2,093 & 29 & 7 & 76 & 6,566 \\
\hline Total & 111 & 358 & 48 & 75 & 40 & 23 & 614 & 744 & 64 & 209 & 588 & 4,920 & 2,141 & 60 & 14 & 90 & 10,099 \\
\hline
\end{tabular}


Table 3.9. Distribution of Hispanic and Latino Population within 80 km (50 mi) of the Hanford 100 K Area Meteorological Station

\begin{tabular}{|c|c|c|c|c|c|c|c|c|c|c|c|c|c|c|c|c|c|}
\hline & \multicolumn{17}{|c|}{ Direction } \\
\hline $\begin{array}{l}\text { Distance } \\
\text { from } \\
\text { Reference } \\
\text { Point } \\
\text { (Miles) } \\
\end{array}$ & $\mathbf{N}$ & NNE & NE & ENE & $\mathbf{E}$ & ESE & SE & SSE & $\mathbf{S}$ & SSW & SW & WSW & $\mathbf{W}$ & WNW & NW & NNW & Total \\
\hline 0-1mi & 0 & 0 & 0 & 0 & 0 & 0 & 0 & 0 & 0 & 0 & 0 & 0 & 0 & 0 & 0 & 0 & 0 \\
\hline 1-2 & 0 & 0 & 0 & 0 & 0 & 0 & 0 & 0 & 0 & 0 & 0 & 0 & 0 & 0 & 0 & 0 & $\mathbf{0}$ \\
\hline $2-3$ & 0 & 0 & 0 & 0 & 0 & 0 & 0 & 0 & 0 & 0 & 0 & 0 & 0 & 0 & 0 & 0 & $\mathbf{0}$ \\
\hline $3-4$ & 0 & 0 & 0 & 0 & 0 & 0 & 0 & 0 & 0 & 0 & 0 & 0 & 0 & 0 & 0 & 0 & $\mathbf{0}$ \\
\hline $4-5$ & 0 & 0 & 0 & 0 & 0 & 0 & 0 & 0 & 0 & 0 & 0 & 0 & 0 & 0 & 0 & 0 & $\mathbf{0}$ \\
\hline 5-6 & 0 & 0 & 0 & 0 & 0 & 0 & 0 & 0 & 0 & 0 & 0 & 0 & 1 & 1 & 1 & 0 & 3 \\
\hline $6-7$ & 0 & 0 & 0 & 0 & 0 & 0 & 0 & 0 & 0 & 0 & 0 & 0 & 2 & 3 & 5 & 6 & 16 \\
\hline $7-8$ & 3 & 1 & 0 & 0 & 0 & 0 & 0 & 0 & 0 & 0 & 0 & 0 & 3 & 5 & 21 & 17 & 50 \\
\hline $8-9$ & 10 & 8 & 0 & 0 & 0 & 0 & 0 & 0 & 0 & 0 & 0 & 0 & 6 & 13 & 45 & 30 & 112 \\
\hline 9-10 & 8 & 8 & 0 & 0 & 1 & 0 & 0 & 0 & 0 & 0 & 0 & 0 & 9 & 12 & 38 & 12 & 88 \\
\hline 10-12 & 1 & 1 & 0 & 0 & 4 & 2 & 0 & 0 & 0 & 0 & 0 & 0 & 16 & 30 & 42 & 1 & 97 \\
\hline $12-14$ & 18 & 12 & 7 & 7 & 13 & 2 & 0 & 0 & 0 & 0 & 3 & 2 & 11 & 74 & 4 & 6 & 159 \\
\hline 14-16 & 99 & 50 & 52 & 24 & 23 & 5 & 0 & 0 & 0 & 0 & 1 & 7 & 395 & 80 & 0 & 29 & 765 \\
\hline $16-18$ & 1,550 & 107 & 124 & 63 & 48 & 18 & 0 & 0 & 0 & 0 & 1 & 0 & 197 & 2,667 & 39 & 67 & 4,881 \\
\hline $18-20$ & 149 & 141 & 210 & 178 & 44 & 331 & 9 & 0 & 1 & 1 & 0 & 0 & 1 & 299 & 114 & 72 & 1,550 \\
\hline 20-25 & 104 & 134 & 266 & 6,410 & 91 & 974 & 236 & 28 & 143 & 12 & 7 & 0 & 0 & 9 & 346 & 160 & 8,920 \\
\hline 25-30 & 3 & 19 & 106 & 113 & 307 & 193 & 319 & 1,636 & 1,108 & 1,033 & 962 & 10 & 0 & 0 & 56 & 108 & 5,973 \\
\hline $30-35$ & 60 & 1,664 & 1,981 & 30 & 1,252 & 34 & 1,009 & 1,542 & 15 & 9,958 & 13,957 & 350 & 1 & 0 & 14 & 611 & 32,478 \\
\hline $35-40$ & 204 & 4,879 & 119 & 31 & 18 & 15 & 22,393 & 3,939 & 6 & 2,225 & 2,901 & 6,205 & 786 & 7 & 6 & 139 & 43,873 \\
\hline $40-50$ & 1,067 & 130 & 21 & 20 & 37 & 781 & 1,062 & 103 & 65 & 69 & 42 & 11,189 & 30,208 & 272 & 6 & 4,011 & 49,083 \\
\hline Total & 3,276 & 7,154 & 2,886 & 6,876 & 1,838 & 2,355 & 25,028 & 7,248 & 1,338 & 13,298 & 17,874 & 17,763 & 31,636 & 3,472 & 737 & 5,269 & 148,048 \\
\hline
\end{tabular}


Table 3.10. Distribution of Total Minority Population within 80 km (50 mi) of the Hanford 100 K Area Meteorological Station

\begin{tabular}{|c|c|c|c|c|c|c|c|c|c|c|c|c|c|c|c|c|c|}
\hline & \multicolumn{17}{|c|}{ Direction } \\
\hline $\begin{array}{l}\text { Distance } \\
\text { from } \\
\text { Reference } \\
\text { Point } \\
\text { (Miles) }\end{array}$ & $\mathbf{N}$ & NNE & NE & ENE & $\mathbf{E}$ & ESE & SE & SSE & $\mathbf{S}$ & SSW & SW & WSW & W & WNW & NW & NNW & Total \\
\hline 0-1mi & 0 & 0 & 0 & 0 & 0 & 0 & 0 & 0 & 0 & 0 & 0 & 0 & 0 & 0 & 0 & 0 & $\mathbf{0}$ \\
\hline $1-2$ & 0 & 0 & 0 & 0 & 0 & 0 & 0 & 0 & 0 & 0 & 0 & 0 & 0 & 0 & 0 & 0 & 0 \\
\hline $2-3$ & 0 & 0 & 0 & 0 & 0 & 0 & 0 & 0 & 0 & 0 & 0 & 0 & 0 & 0 & 0 & 0 & $\mathbf{0}$ \\
\hline $3-4$ & 0 & 0 & 0 & 0 & 0 & 0 & 0 & 0 & 0 & 0 & 0 & 0 & 0 & 0 & 0 & 0 & $\mathbf{0}$ \\
\hline $4-5$ & 0 & 0 & 0 & 0 & 0 & 0 & 0 & 0 & 0 & 0 & 0 & 0 & 0 & 0 & 0 & 0 & $\mathbf{0}$ \\
\hline $5-6$ & 0 & 0 & 0 & 0 & 0 & 0 & 0 & 0 & 0 & 0 & 0 & 0 & 1 & 1 & 1 & 0 & 3 \\
\hline $6-7$ & 0 & 0 & 0 & 0 & 0 & 0 & 0 & 0 & 0 & 0 & 0 & 0 & 2 & 4 & 6 & 6 & 18 \\
\hline $7-8$ & 3 & 1 & 0 & 0 & 0 & 0 & 0 & 0 & 0 & 0 & 0 & 0 & 3 & 5 & 21 & 17 & 50 \\
\hline $8-9$ & 10 & 8 & 0 & 0 & 0 & 0 & 0 & 0 & 0 & 0 & 0 & 0 & 6 & 13 & 45 & 30 & 112 \\
\hline 9-10 & 9 & 8 & 0 & 0 & 1 & 1 & 0 & 0 & 0 & 0 & 0 & 0 & 9 & 13 & 38 & 12 & 91 \\
\hline $10-12$ & 2 & 1 & 0 & 1 & 4 & 2 & 0 & 0 & 0 & 0 & 0 & 0 & 19 & 35 & 42 & 2 & 108 \\
\hline $12-14$ & 23 & 13 & 10 & 8 & 14 & 3 & 0 & 0 & 0 & 0 & 3 & 2 & 19 & 74 & 4 & 7 & 180 \\
\hline 14-16 & 101 & 51 & 64 & 25 & 23 & 6 & 0 & 0 & 0 & 0 & 1 & 7 & 411 & 80 & 0 & 32 & 801 \\
\hline $16-18$ & 1,583 & 108 & 126 & 64 & 50 & 21 & 2 & 0 & 0 & 0 & 1 & 0 & 207 & 2,720 & 39 & 68 & 4,989 \\
\hline $18-20$ & 154 & 142 & 217 & 188 & 48 & 338 & 9 & 0 & 1 & 1 & 0 & 0 & 1 & 301 & 115 & 76 & 1,591 \\
\hline $20-25$ & 108 & 149 & 274 & 6,565 & 96 & 1,001 & 250 & 56 & 160 & 12 & 12 & 2 & 0 & 9 & 351 & 170 & 9,215 \\
\hline $25-30$ & 4 & 30 & 127 & 113 & 328 & 199 & 358 & 4,110 & 1,276 & 1,089 & 1,006 & 11 & 0 & 0 & 58 & 123 & 8,832 \\
\hline 30-35 & 73 & 2,142 & 2,034 & 32 & 1,563 & 37 & 1,333 & 3,307 & 15 & 10,422 & 14,443 & 410 & 5 & 0 & 14 & 644 & 36,474 \\
\hline $35-40$ & 249 & 6,314 & 136 & 40 & 21 & 15 & 25,471 & 6,141 & 8 & 2,279 & 3,275 & 7,288 & 916 & 13 & 6 & 181 & 52,353 \\
\hline $40-50$ & 1,464 & 175 & 28 & 34 & 49 & 809 & 1,405 & 135 & 68 & 82 & 144 & 15,889 & 37,132 & 400 & 23 & 4,147 & 61,984 \\
\hline Total & 3,783 & 9,142 & 3,016 & 7,070 & 2,197 & 2,432 & 28,828 & 13,749 & 1,528 & 13,885 & 18,885 & 23,609 & 38,731 & 3,668 & 763 & 5,515 & 176,801 \\
\hline
\end{tabular}


Table 3.11. Distribution of Low-Income Population within 80 km (50 mi) of the Hanford $100 \mathrm{~K}$ Area Meteorological Station

\begin{tabular}{|c|c|c|c|c|c|c|c|c|c|c|c|c|c|c|c|c|c|}
\hline & \multicolumn{17}{|c|}{ Direction } \\
\hline $\begin{array}{l}\text { Distance } \\
\text { from } \\
\text { Reference } \\
\text { Point } \\
\text { (Miles) } \\
\end{array}$ & $\mathbf{N}$ & NNE & NE & ENE & $\mathbf{E}$ & ESE & SE & SSE & $\mathbf{S}$ & SSW & SW & WSW & $\mathbf{W}$ & WNW & NW & NNW & Total \\
\hline 0-1mi & 0 & 0 & 0 & 0 & 0 & 0 & 0 & 0 & 0 & 0 & 0 & 0 & 0 & 0 & 0 & 0 & 0 \\
\hline $1-2$ & 0 & 0 & 0 & 0 & 0 & 0 & 0 & 0 & 0 & 0 & 0 & 0 & 0 & 0 & 0 & 0 & 0 \\
\hline $2-3$ & 0 & 0 & 0 & 0 & 0 & 0 & 0 & 0 & 0 & 0 & 0 & 0 & 0 & 0 & 0 & 0 & 0 \\
\hline $3-4$ & 0 & 0 & 0 & 0 & 0 & 0 & 0 & 0 & 0 & 0 & 0 & 0 & 0 & 0 & 0 & 0 & 0 \\
\hline $4-5$ & 0 & 0 & 0 & 0 & 0 & 0 & 0 & 0 & 0 & 0 & 0 & 0 & 0 & 0 & 0 & 0 & 0 \\
\hline $5-6$ & 0 & 0 & 0 & 0 & 0 & 0 & 0 & 0 & 0 & 0 & 0 & 0 & 0 & 0 & 0 & 0 & 0 \\
\hline 6-7 & 0 & 0 & 0 & 0 & 0 & 0 & 0 & 0 & 0 & 0 & 0 & 0 & 1 & 1 & 2 & 2 & 6 \\
\hline $7-8$ & 1 & 0 & 0 & 0 & 0 & 0 & 0 & 0 & 0 & 0 & 0 & 0 & 1 & 2 & 8 & 6 & 18 \\
\hline 8-9 & 3 & 2 & 0 & 0 & 0 & 0 & 0 & 0 & 0 & 0 & 0 & 0 & 2 & 4 & 14 & 9 & 34 \\
\hline 9-10 & 3 & 2 & 0 & 0 & 1 & 0 & 0 & 0 & 0 & 0 & 0 & 0 & 3 & 4 & 11 & 3 & 27 \\
\hline $10-12$ & 3 & 1 & 0 & 1 & 1 & 1 & 0 & 0 & 0 & 0 & 0 & 0 & 7 & 16 & 11 & 3 & 44 \\
\hline $12-14$ & 9 & 2 & 1 & 2 & 3 & 2 & 0 & 0 & 0 & 0 & 1 & 2 & 12 & 29 & 4 & 6 & 73 \\
\hline 14-16 & 20 & 12 & 10 & 3 & 6 & 3 & 0 & 0 & 0 & 0 & 1 & 2 & 126 & 26 & 0 & 12 & 221 \\
\hline $16-18$ & 525 & 27 & 22 & 10 & 10 & 8 & 0 & 0 & 0 & 0 & 1 & 1 & 138 & 673 & 18 & 37 & 1,470 \\
\hline $18-20$ & 37 & 43 & 72 & 77 & 32 & 111 & 3 & 0 & 0 & 1 & 0 & 0 & 0 & 86 & 54 & 34 & 550 \\
\hline 20-25 & 32 & 74 & 84 & 2,111 & 81 & 398 & 66 & 44 & 67 & 4 & 9 & 2 & 0 & 1 & 177 & 100 & 3,250 \\
\hline $25-30$ & 10 & 22 & 34 & 11 & 163 & 62 & 145 & 2,616 & 759 & 348 & 440 & 5 & 0 & 0 & 25 & 117 & 4,757 \\
\hline 30-35 & 48 & 1,244 & 638 & 4 & 579 & 11 & 109 & 1,718 & 11 & 3,777 & 6,295 & 283 & 5 & 0 & 10 & 310 & 15,042 \\
\hline $35-40$ & 126 & 3,757 & 53 & 19 & 13 & 6 & 11,177 & 3,427 & 13 & 819 & 1,295 & 2,956 & 334 & 9 & 32 & 113 & 24,149 \\
\hline $40-50$ & 1,371 & 116 & 23 & 22 & 63 & 195 & 704 & 18 & 33 & 33 & 72 & 5,680 & 20,721 & 539 & 21 & 1,328 & 30,939 \\
\hline Total & 2,188 & 5,302 & 937 & 2,260 & 952 & 797 & 12,204 & 7,823 & 883 & 4,982 & 8,114 & 8,931 & 21,350 & 1,390 & 387 & 2,080 & 80,580 \\
\hline
\end{tabular}




\subsection{Area}

The Hanford Meteorological Station is near the center of the Hanford Site. At the time of the 2000 Census there were about 486,300 people residing within $80 \mathrm{~km}(50 \mathrm{mi})$ of this location. Based on census block data, about 180,000 (37\%) of those residents were minorities. The Hispanic and Latino population (all races) was the single largest minority group, with 150,800 members (31\% of the total population). There were 10,400 Native Americans (2\% of the total). There were an estimated 81,300 low-income individuals (about $17 \%$ of the population).

Figures 3.14 to 3.19 and Tables 3.12 to 3.16 show population distributions for total, minority, and low-income populations living within a 50-mile radius of the Hanford Meteorological Station in the 200 Area of the Hanford Site.

Because of its location near the center of the Hanford Site, the resident population within 10 miles of the Hanford Meteorological Station is very small (estimated as 23 individuals). About 800 people live within 15 miles, and 10,400 people live within 20 miles. There are about 56,000 people living between 20 and 25 miles from the 200 Area Meteorological Station, located mostly to the southwest and the southeast. There are about 1,300 Native Americans living between 20 and 40 miles to the southeast, and about 8,000 Native Americans living 25 to 50 miles to the west and west-southwest. Substantial Hispanic and Latino populations are located 18 to 20 miles to the northwest (2,600 individuals), 25 to 30 miles to the northeast (6,000 individuals) and southwest (18,200 individuals), 30 to 35 miles to the southeast (26,000 individuals), and 40 to 50 miles to the north-northeast (6,300 individuals) and due west (31,500 individuals). 


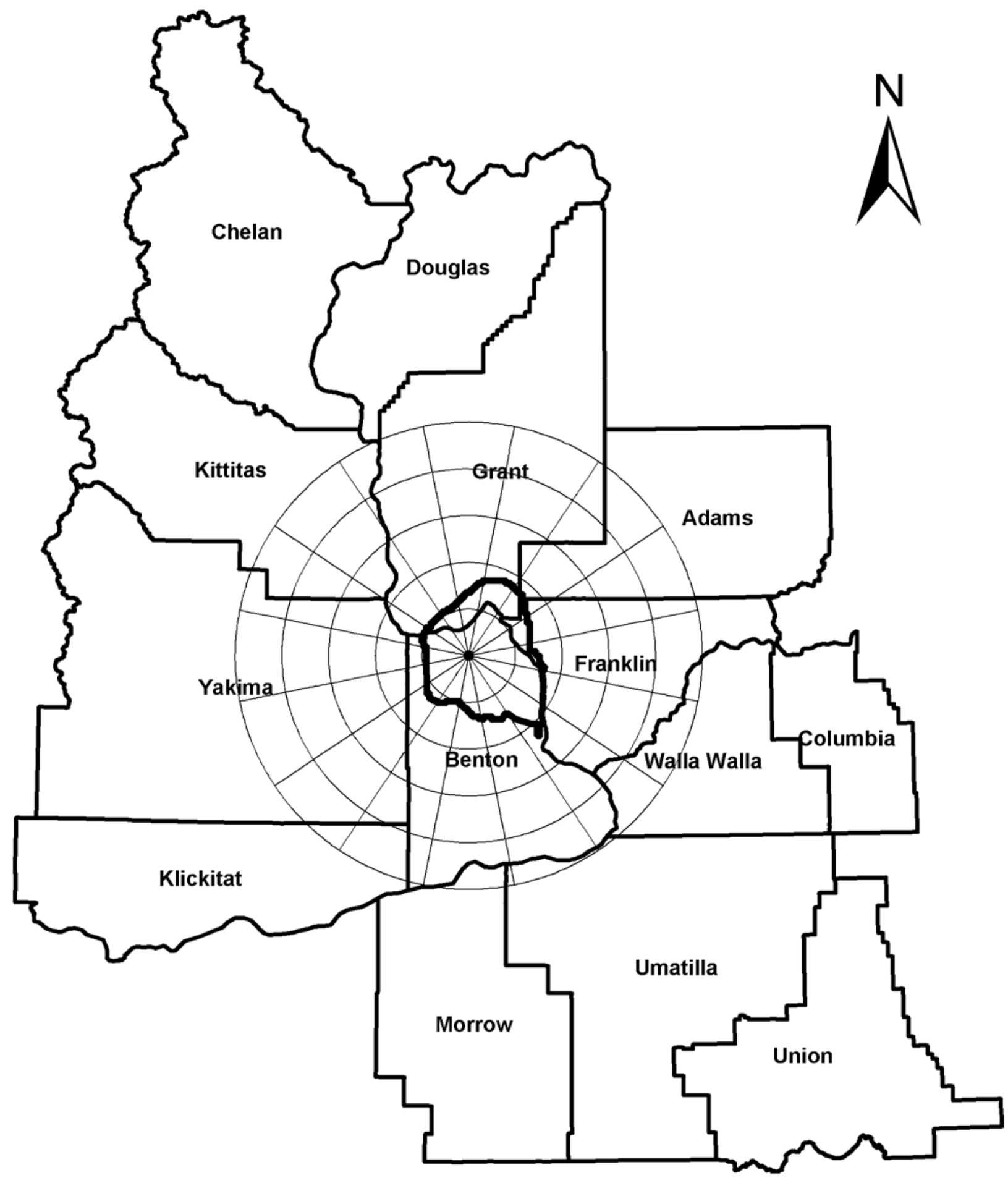

Figure 3.14. Map of 80-km (50-mi) Region Surrounding the 200 Area Hanford Meteorological Station 


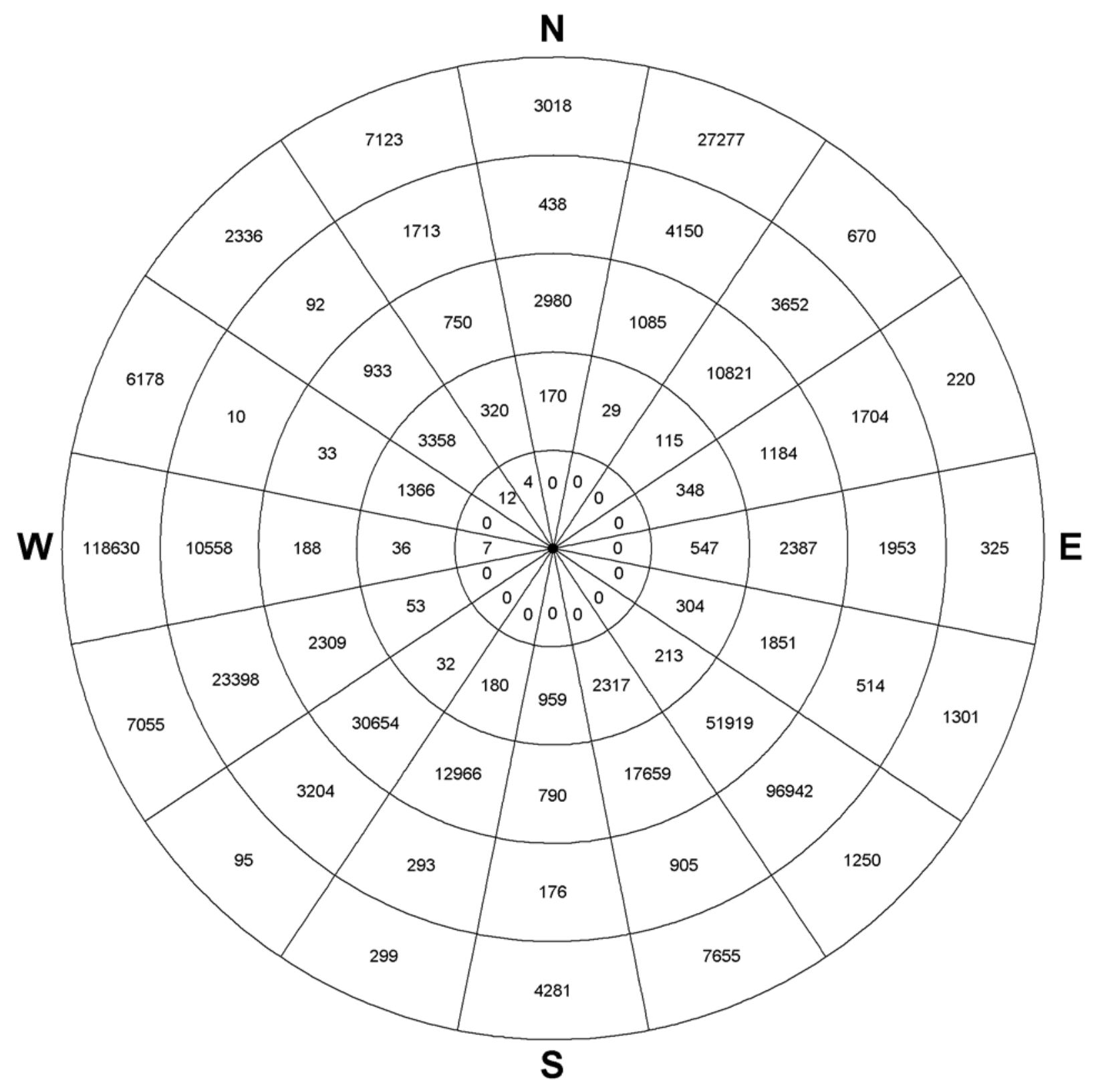

Figure 3.15. Map of Total Resident Population within $80 \mathrm{~km}(50 \mathrm{mi})$ of the 200 Area Hanford Meteorological Station, in 16-km (10-mi) Increments by Distance and Direction 


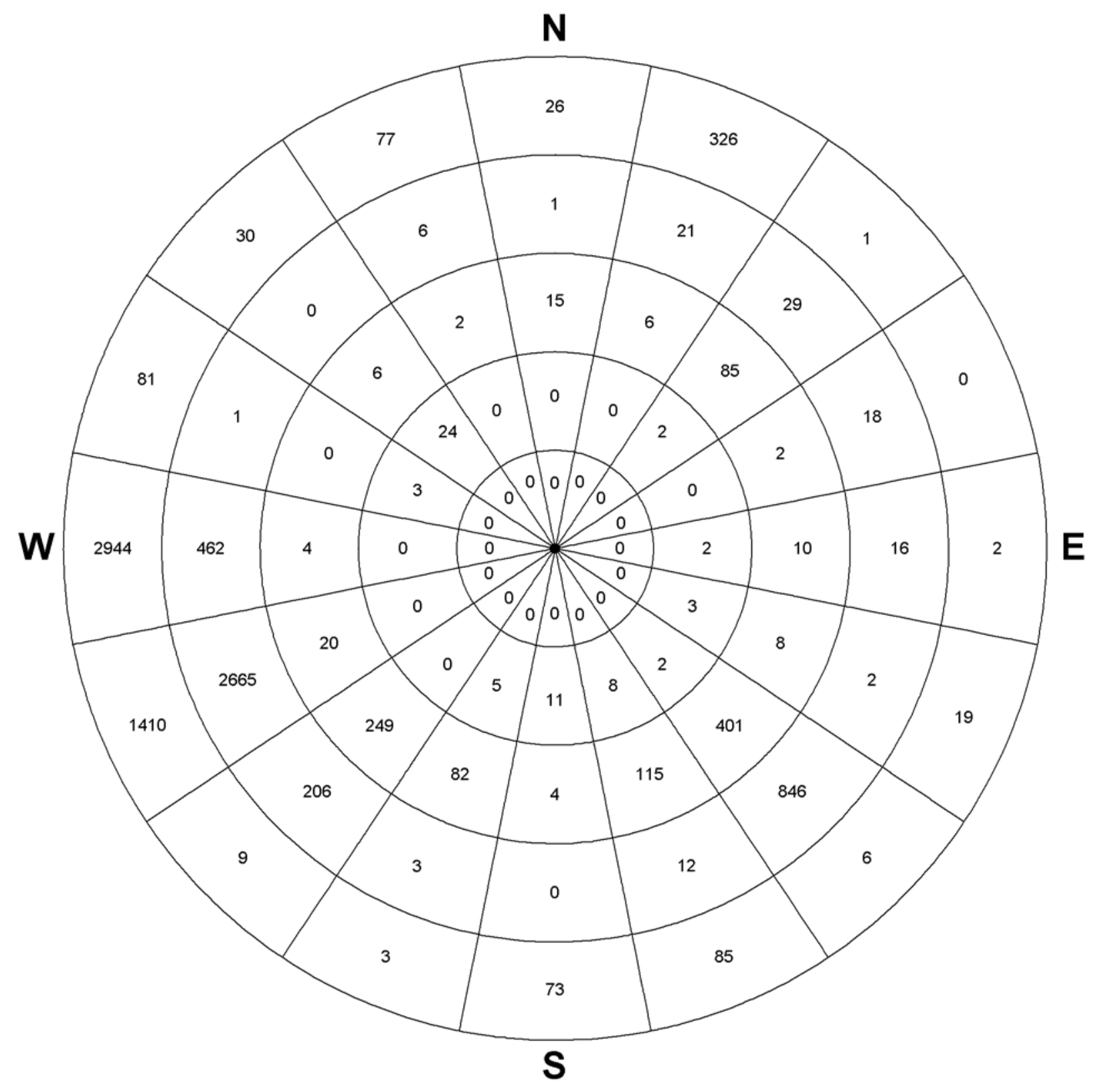

Figure 3.16. Map of Native American Population within $80 \mathrm{~km}(50 \mathrm{mi})$ of the 200 Area Hanford Meteorological Station, in 16-km (10-mi) Increments by Distance and Direction 


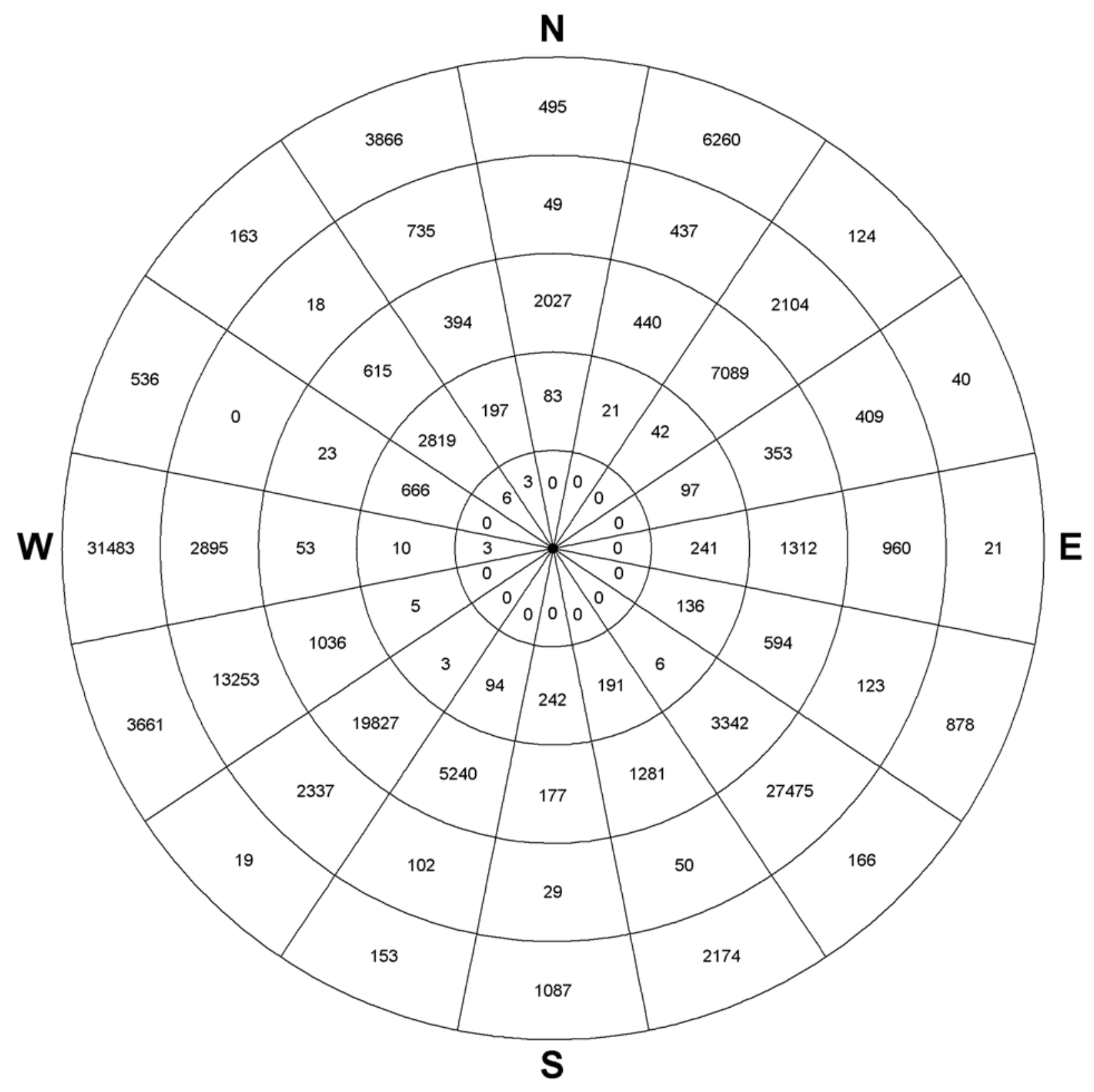

Figure 3.17. Map of Hispanic and Latino Population within $80 \mathrm{~km}(50 \mathrm{mi})$ of the 200 Area Hanford Meteorological Station, in 16-km (10-mi) Increments by Distance and Direction 


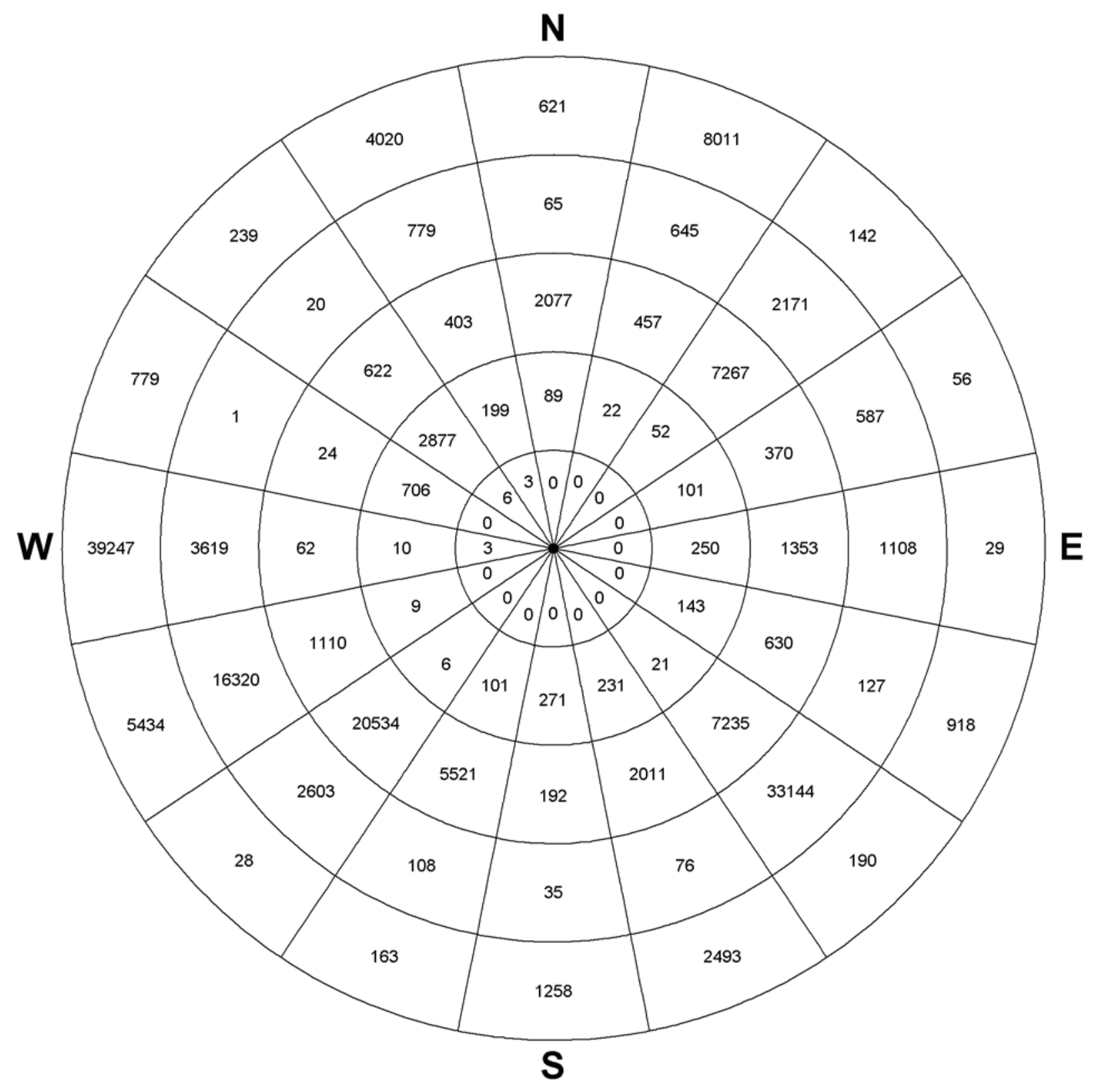

Figure 3.18. Map of Total Minority Population within $80 \mathrm{~km}(50 \mathrm{mi})$ of the 200 Area Hanford Meteorological Station, in 16-km (10-mi) Increments by Distance and Direction 


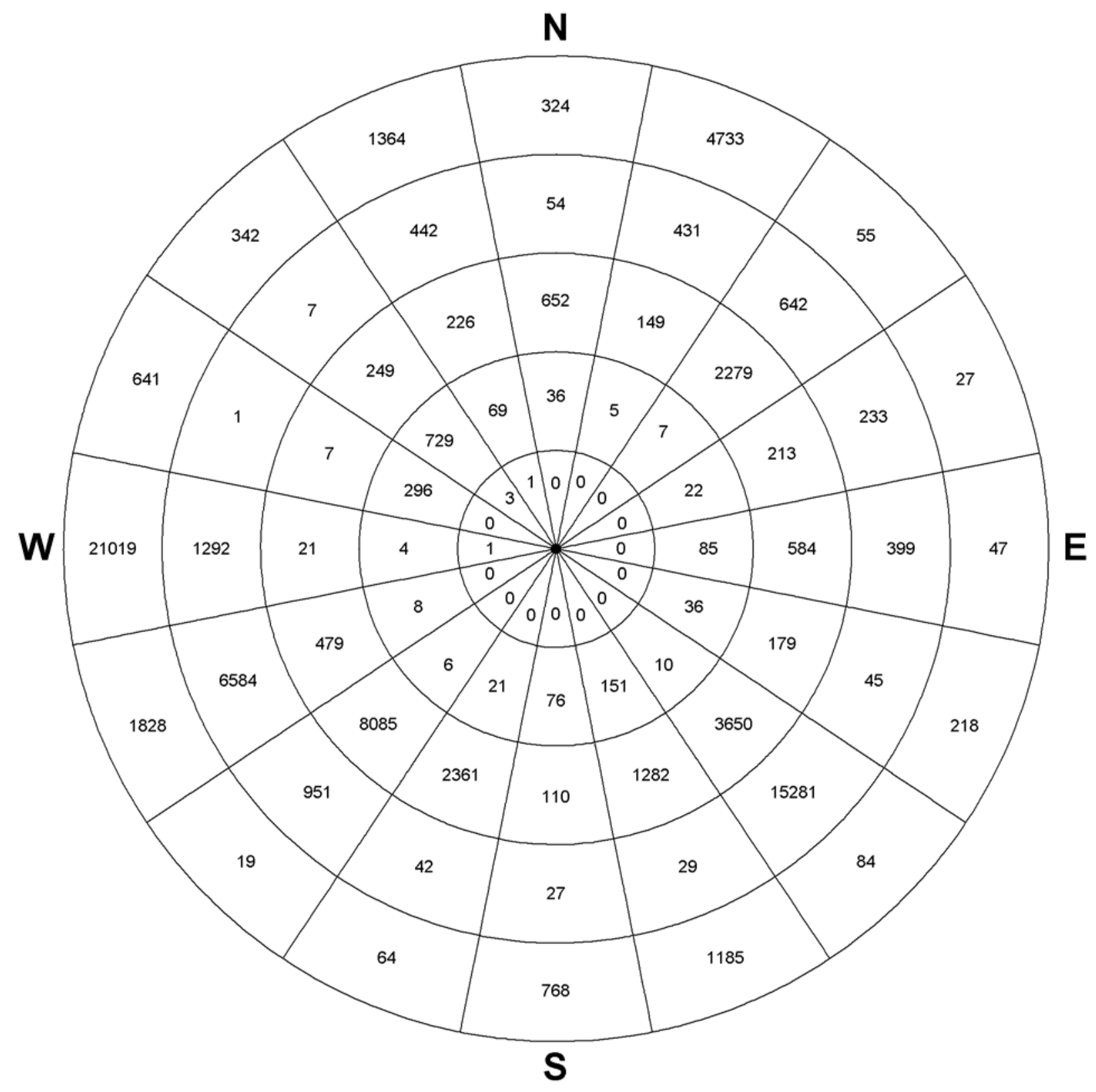

Figure 3.19. Map of Low-Income Population within $80 \mathrm{~km}$ (50 mi) of the 200 Area Hanford Meteorological Station, in 16-km (10-mi) Increments by Distance and Direction 
Table 3.12. Distribution of Total Resident Population within $80 \mathrm{~km}(50 \mathrm{mi})$ of the 200 Area Hanford Meteorological Station

\begin{tabular}{|c|c|c|c|c|c|c|c|c|c|c|c|c|c|c|c|c|c|}
\hline \multirow[b]{2}{*}{$\begin{array}{l}\text { Distance } \\
\text { from } \\
\text { Reference } \\
\text { Point } \\
\text { (Miles) } \\
\end{array}$} & \multicolumn{17}{|c|}{ Direction } \\
\hline & $\mathbf{N}$ & NNE & NE & ENE & $\mathbf{E}$ & ESE & SE & SSE & $\mathbf{S}$ & SSW & SW & WSW & $\mathbf{W}$ & WNW & NW & NNW & Total \\
\hline 0-1mi & 0 & 0 & 0 & 0 & 0 & 0 & 0 & 0 & 0 & 0 & 0 & 0 & 0 & 0 & 0 & 0 & $\mathbf{0}$ \\
\hline $1-2$ & 0 & 0 & 0 & 0 & 0 & 0 & 0 & 0 & 0 & 0 & 0 & 0 & 0 & 0 & 0 & 0 & $\mathbf{0}$ \\
\hline $2-3$ & 0 & 0 & 0 & 0 & 0 & 0 & 0 & 0 & 0 & 0 & 0 & 0 & 0 & 0 & 0 & 0 & $\mathbf{0}$ \\
\hline $3-4$ & 0 & 0 & 0 & 0 & 0 & 0 & 0 & 0 & 0 & 0 & 0 & 0 & 0 & 0 & 0 & 0 & $\mathbf{0}$ \\
\hline $4-5$ & 0 & 0 & 0 & 0 & 0 & 0 & 0 & 0 & 0 & 0 & 0 & 0 & 0 & 0 & 0 & 0 & $\mathbf{0}$ \\
\hline 5-6 & 0 & 0 & 0 & 0 & 0 & 0 & 0 & 0 & 0 & 0 & 0 & 0 & 0 & 0 & 0 & 0 & $\mathbf{0}$ \\
\hline $6-7$ & 0 & 0 & 0 & 0 & 0 & 0 & 0 & 0 & 0 & 0 & 0 & 0 & 0 & 0 & 0 & 0 & $\mathbf{0}$ \\
\hline $7-8$ & 0 & 0 & 0 & 0 & 0 & 0 & 0 & 0 & 0 & 0 & 0 & 0 & 0 & 0 & 0 & 0 & $\mathbf{0}$ \\
\hline $8-9$ & 0 & 0 & 0 & 0 & 0 & 0 & 0 & 0 & 0 & 0 & 0 & 0 & 0 & 0 & 4 & 1 & 5 \\
\hline 9-10 & 0 & 0 & 0 & 0 & 0 & 0 & 0 & 0 & 0 & 0 & 0 & 0 & 7 & 0 & 8 & 3 & 18 \\
\hline 10-12 & 0 & 0 & 1 & 15 & 2 & 0 & 0 & 0 & 0 & 1 & 0 & 7 & 24 & 9 & 38 & 21 & 118 \\
\hline $12-14$ & 23 & 0 & 3 & 29 & 12 & 0 & 0 & 3 & 2 & 9 & 0 & 13 & 7 & 32 & 52 & 127 & 312 \\
\hline 14-16 & 58 & 12 & 10 & 45 & 40 & 4 & 15 & 116 & 4 & 12 & 0 & 7 & 3 & 66 & 143 & 123 & 658 \\
\hline 16-18 & 26 & 7 & 17 & 113 & 169 & 44 & 114 & 462 & 112 & 16 & 8 & 13 & 1 & 1,075 & 166 & 28 & 2,371 \\
\hline 18-20 & 63 & 10 & 84 & 146 & 324 & 256 & 84 & 1,736 & 841 & 142 & 24 & 13 & 1 & 184 & 2,959 & 21 & 6,888 \\
\hline $20-25$ & 2,484 & 393 & 2,035 & 665 & 1,636 & 1,139 & 26,281 & 12,743 & 750 & 2,972 & 3,276 & 340 & 14 & 33 & 805 & 329 & 55,895 \\
\hline $25-30$ & 496 & 692 & 8,786 & 519 & 751 & 712 & 25,638 & 4,916 & 40 & 9,994 & 27,378 & 1,969 & 174 & 0 & 128 & 421 & 82,614 \\
\hline $30-35$ & 66 & 379 & 503 & 356 & 46 & 271 & 83,468 & 886 & 126 & 262 & 3,060 & 8,590 & 822 & 0 & 88 & 426 & 99,349 \\
\hline $35-40$ & 372 & 3,771 & 3,149 & 1,348 & 1,907 & 243 & 13,474 & 19 & 50 & 31 & 144 & 14,808 & 9,736 & 10 & 4 & 1,287 & 50,353 \\
\hline $40-50$ & 3,018 & 27,277 & 670 & 220 & 325 & 1,301 & 1,250 & 7,655 & 4,281 & 299 & 95 & 7,055 & 118,630 & 6,178 & 2,336 & 7,123 & 187,713 \\
\hline Total & 6,606 & 32,541 & 15,258 & 3,456 & 5,212 & 3,970 & 150,324 & 28,536 & 6,206 & 13,738 & 33,985 & 32,815 & 129,419 & 7,587 & 6,731 & 9,910 & 486,294 \\
\hline
\end{tabular}


Table 3.13. Distribution of Native American Population within 80 km (50 mi) of the 200 Area Hanford Meteorological Station

\begin{tabular}{|c|c|c|c|c|c|c|c|c|c|c|c|c|c|c|c|c|c|}
\hline & \multicolumn{17}{|c|}{ Direction } \\
\hline $\begin{array}{l}\text { Distance } \\
\text { from } \\
\text { Reference } \\
\text { Point } \\
\text { (Miles) } \\
\end{array}$ & $\mathbf{N}$ & NNE & NE & ENE & $\mathbf{E}$ & ESE & SE & SSE & $\mathbf{S}$ & SSW & SW & WSW & $\mathbf{W}$ & WNW & NW & NNW & Total \\
\hline o-1mi & 0 & 0 & 0 & 0 & 0 & 0 & 0 & 0 & 0 & 0 & 0 & 0 & 0 & 0 & 0 & 0 & $\mathbf{0}$ \\
\hline 1-2 & 0 & 0 & 0 & 0 & 0 & 0 & 0 & 0 & 0 & 0 & 0 & 0 & 0 & 0 & 0 & 0 & $\mathbf{0}$ \\
\hline $2-3$ & 0 & 0 & 0 & 0 & 0 & 0 & 0 & 0 & 0 & 0 & 0 & 0 & 0 & 0 & 0 & 0 & $\mathbf{0}$ \\
\hline $3-4$ & 0 & 0 & 0 & 0 & 0 & 0 & 0 & 0 & 0 & 0 & 0 & 0 & 0 & 0 & 0 & 0 & $\mathbf{0}$ \\
\hline 4-5 & 0 & 0 & 0 & 0 & 0 & 0 & 0 & 0 & 0 & 0 & 0 & 0 & 0 & 0 & 0 & 0 & $\mathbf{0}$ \\
\hline $5-6$ & 0 & 0 & 0 & 0 & 0 & 0 & 0 & 0 & 0 & 0 & 0 & 0 & 0 & 0 & 0 & 0 & $\mathbf{0}$ \\
\hline 6-7 & 0 & 0 & 0 & 0 & 0 & 0 & 0 & 0 & 0 & 0 & 0 & 0 & 0 & 0 & 0 & 0 & $\mathbf{0}$ \\
\hline $7-8$ & 0 & 0 & 0 & 0 & 0 & 0 & 0 & 0 & 0 & 0 & 0 & 0 & 0 & 0 & 0 & 0 & $\mathbf{0}$ \\
\hline $8-9$ & 0 & 0 & 0 & 0 & 0 & 0 & 0 & 0 & 0 & 0 & 0 & 0 & 0 & 0 & 0 & 0 & $\mathbf{0}$ \\
\hline 9-10 & 0 & 0 & 0 & 0 & 0 & 0 & 0 & 0 & 0 & 0 & 0 & 0 & 0 & 0 & 0 & 0 & $\mathbf{0}$ \\
\hline $10-12$ & 0 & 0 & 0 & 0 & 0 & 0 & 0 & 0 & 0 & 0 & 0 & 0 & 0 & 0 & 0 & 0 & 0 \\
\hline $12-14$ & 0 & 0 & 0 & 0 & 0 & 0 & 0 & 0 & 0 & 0 & 0 & 0 & 0 & 0 & 3 & 0 & 3 \\
\hline 14-16 & 0 & 0 & 0 & 0 & 0 & 0 & 0 & 0 & 2 & 0 & 0 & 0 & 0 & 0 & 2 & 0 & 4 \\
\hline $16-18$ & 0 & 0 & 0 & 0 & 1 & 0 & 1 & 1 & 3 & 0 & 0 & 0 & 0 & 2 & 0 & 0 & 8 \\
\hline $18-20$ & 0 & 0 & 2 & 0 & 1 & 3 & 1 & 7 & 6 & 5 & 0 & 0 & 0 & 1 & 19 & 0 & 45 \\
\hline $20-25$ & 12 & 2 & 12 & 1 & 6 & 7 & 230 & 96 & 4 & 6 & 23 & 0 & 0 & 0 & 3 & 1 & 403 \\
\hline $25-30$ & 3 & 4 & 73 & 1 & 4 & 1 & 171 & 19 & 0 & 76 & 226 & 20 & 4 & 0 & 3 & 1 & 606 \\
\hline $30-35$ & 0 & 1 & 1 & 0 & 0 & 1 & 717 & 12 & 0 & 0 & 156 & 364 & 14 & 0 & 0 & 2 & 1,268 \\
\hline $35-40$ & 1 & 20 & 28 & 18 & 16 & 1 & 129 & 0 & 0 & 3 & 50 & 2,301 & 448 & 1 & 0 & 4 & 3,020 \\
\hline $40-50$ & 26 & 326 & 1 & 0 & 2 & 19 & 6 & 85 & 73 & 3 & 9 & 1,410 & 2,944 & 81 & 30 & 77 & 5,092 \\
\hline Total & 42 & 353 & 117 & 20 & 30 & 32 & 1,255 & 220 & 88 & 93 & 464 & 4,095 & 3,410 & 85 & 60 & 85 & 10,449 \\
\hline
\end{tabular}


Table 3.14. Distribution of Hispanic and Latino Population within $80 \mathrm{~km}(50 \mathrm{mi})$ of the 200 Area Hanford Meteorological Station

\begin{tabular}{|c|c|c|c|c|c|c|c|c|c|c|c|c|c|c|c|c|c|}
\hline & \multicolumn{17}{|c|}{ Direction } \\
\hline $\begin{array}{l}\text { Distance } \\
\text { from } \\
\text { Reference } \\
\text { Point } \\
\text { (Miles) }\end{array}$ & $\mathbf{N}$ & NNE & NE & ENE & $\mathbf{E}$ & ESE & SE & SSE & $\mathbf{S}$ & SSW & SW & WSW & W & WNW & NW & NNW & Total \\
\hline 0-1mi & 0 & 0 & 0 & 0 & 0 & 0 & 0 & 0 & 0 & 0 & 0 & 0 & 0 & 0 & 0 & 0 & $\mathbf{0}$ \\
\hline 1-2 & 0 & 0 & 0 & 0 & 0 & 0 & 0 & 0 & 0 & 0 & 0 & 0 & 0 & 0 & 0 & 0 & $\mathbf{0}$ \\
\hline $2-3$ & 0 & 0 & 0 & 0 & 0 & 0 & 0 & 0 & 0 & 0 & 0 & 0 & 0 & 0 & 0 & 0 & $\mathbf{0}$ \\
\hline $3-4$ & 0 & 0 & 0 & 0 & 0 & 0 & 0 & 0 & 0 & 0 & 0 & 0 & 0 & 0 & 0 & 0 & $\mathbf{0}$ \\
\hline $4-5$ & 0 & 0 & 0 & 0 & 0 & 0 & 0 & 0 & 0 & 0 & 0 & 0 & 0 & 0 & 0 & 0 & $\mathbf{0}$ \\
\hline $5-6$ & 0 & 0 & 0 & 0 & 0 & 0 & 0 & 0 & 0 & 0 & 0 & 0 & 0 & 0 & 0 & 0 & $\mathbf{0}$ \\
\hline 6-7 & 0 & 0 & 0 & 0 & 0 & 0 & 0 & 0 & 0 & 0 & 0 & 0 & 0 & 0 & 0 & 0 & $\mathbf{0}$ \\
\hline $7-8$ & 0 & 0 & 0 & 0 & 0 & 0 & 0 & 0 & 0 & 0 & 0 & 0 & 0 & 0 & 0 & 0 & $\mathbf{0}$ \\
\hline $8-9$ & 0 & 0 & 0 & 0 & 0 & 0 & 0 & 0 & 0 & 0 & 0 & 0 & 0 & 0 & 2 & 1 & 3 \\
\hline 9-10 & 0 & 0 & 0 & 0 & 0 & 0 & 0 & 0 & 0 & 0 & 0 & 0 & 3 & 0 & 4 & 2 & 9 \\
\hline 10-12 & 0 & 0 & 0 & 2 & 0 & 0 & 0 & 0 & 0 & 0 & 0 & 0 & 8 & 2 & 29 & 11 & 52 \\
\hline $12-14$ & 12 & 0 & 0 & 4 & 1 & 0 & 0 & 1 & 0 & 1 & 0 & 1 & 2 & 4 & 25 & 81 & 132 \\
\hline 14-16 & 51 & 11 & 0 & 11 & 4 & 0 & 0 & 11 & 3 & 4 & 0 & 0 & 0 & 38 & 69 & 91 & 293 \\
\hline 16-18 & 5 & 4 & 1 & 29 & 60 & 16 & 1 & 16 & 38 & 5 & 1 & 1 & 0 & 537 & 109 & 11 & 834 \\
\hline $18-20$ & 15 & 6 & 41 & 51 & 176 & 120 & 5 & 163 & 201 & 84 & 2 & 3 & 0 & 85 & 2,587 & 3 & 3,542 \\
\hline 20-25 & 1,766 & 183 & 1,074 & 217 & 986 & 428 & 1,302 & 1,080 & 177 & 1,394 & 1,587 & 133 & 2 & 23 & 576 & 189 & 11,117 \\
\hline $25-30$ & 261 & 257 & 6,015 & 136 & 326 & 166 & 2,040 & 201 & 0 & 3,846 & 18,240 & 903 & 51 & 0 & 39 & 205 & 32,686 \\
\hline 30-35 & 9 & 32 & 158 & 72 & 5 & 58 & 25,957 & 43 & 17 & 88 & 2,303 & 3,858 & 160 & 0 & 18 & 154 & 32,932 \\
\hline $35-40$ & 40 & 405 & 1,946 & 337 & 955 & 65 & 1,518 & 7 & 12 & 14 & 34 & 9,395 & 2,735 & 0 & 0 & 581 & 18,044 \\
\hline $40-50$ & 495 & 6,260 & 124 & 40 & 21 & 878 & 166 & 2,174 & 1,087 & 153 & 19 & 3,661 & 31,483 & 536 & 163 & 3,866 & 51,126 \\
\hline Total & 2,654 & 7,158 & 9,359 & 899 & 2,534 & 1,731 & 30,989 & 3,696 & 1,535 & 5,589 & 22,186 & 17,955 & 34,444 & 1,225 & 3,621 & 5,195 & 150,770 \\
\hline
\end{tabular}


Table 3.15. Distribution of Total Minority Population within 80 km (50 mi) of the 200 Area Hanford Meteorological Station

\begin{tabular}{|c|c|c|c|c|c|c|c|c|c|c|c|c|c|c|c|c|c|}
\hline & \multicolumn{17}{|c|}{ Direction } \\
\hline $\begin{array}{l}\text { Distance } \\
\text { from } \\
\text { Reference } \\
\text { Point } \\
\text { (Miles) }\end{array}$ & $\mathbf{N}$ & NNE & NE & ENE & $\mathbf{E}$ & ESE & SE & SSE & $\mathbf{S}$ & SSW & SW & WSW & $\mathbf{W}$ & WNW & NW & NNW & Total \\
\hline 0-1mi & 0 & 0 & 0 & 0 & 0 & 0 & 0 & 0 & 0 & 0 & 0 & 0 & 0 & 0 & 0 & 0 & $\mathbf{0}$ \\
\hline $1-2$ & 0 & 0 & 0 & 0 & 0 & 0 & 0 & 0 & 0 & 0 & 0 & 0 & 0 & 0 & 0 & 0 & 0 \\
\hline $2-3$ & 0 & 0 & 0 & 0 & 0 & 0 & 0 & 0 & 0 & 0 & 0 & 0 & 0 & 0 & 0 & 0 & 0 \\
\hline $3-4$ & 0 & 0 & 0 & 0 & 0 & 0 & 0 & 0 & 0 & 0 & 0 & 0 & 0 & 0 & 0 & 0 & $\mathbf{0}$ \\
\hline $4-5$ & 0 & 0 & 0 & 0 & 0 & 0 & 0 & 0 & 0 & 0 & 0 & 0 & 0 & 0 & 0 & 0 & $\mathbf{0}$ \\
\hline $5-6$ & 0 & 0 & 0 & 0 & 0 & 0 & 0 & 0 & 0 & 0 & 0 & 0 & 0 & 0 & 0 & 0 & $\mathbf{0}$ \\
\hline 6-7 & 0 & 0 & 0 & 0 & 0 & 0 & 0 & 0 & 0 & 0 & 0 & 0 & 0 & 0 & 0 & 0 & 0 \\
\hline $7-8$ & 0 & 0 & 0 & 0 & 0 & 0 & 0 & 0 & 0 & 0 & 0 & 0 & 0 & 0 & 0 & 0 & $\mathbf{0}$ \\
\hline $8-9$ & 0 & 0 & 0 & 0 & 0 & 0 & 0 & 0 & 0 & 0 & 0 & 0 & 0 & 0 & 2 & 1 & 3 \\
\hline 9-10 & 0 & 0 & 0 & 0 & 0 & 0 & 0 & 0 & 0 & 0 & 0 & 0 & 3 & 0 & 4 & 2 & 9 \\
\hline 10-12 & 0 & 0 & 0 & 2 & 0 & 0 & 0 & 0 & 0 & 0 & 0 & 0 & 8 & 3 & 30 & 13 & 56 \\
\hline $12-14$ & 12 & 0 & 0 & 4 & 2 & 0 & 0 & 1 & 0 & 1 & 0 & 1 & 2 & 11 & 29 & 81 & 144 \\
\hline 14-16 & 51 & 11 & 1 & 12 & 5 & 2 & 1 & 12 & 3 & 4 & 0 & 1 & 0 & 41 & 71 & 91 & 306 \\
\hline 16-18 & 6 & 4 & 2 & 29 & 63 & 16 & 3 & 24 & 38 & 5 & 2 & 3 & 0 & 561 & 109 & 11 & 876 \\
\hline 18-20 & 20 & 7 & 49 & 54 & 180 & 125 & 17 & 194 & 230 & 91 & 4 & 4 & 0 & 90 & 2,638 & 3 & 3,706 \\
\hline 20-25 & 1,805 & 186 & 1,107 & 223 & 1,013 & 448 & 3,413 & 1,610 & 192 & 1,458 & 1,663 & 139 & 3 & 24 & 579 & 191 & 14,054 \\
\hline $25-30$ & 272 & 271 & 6,160 & 147 & 340 & 182 & 3,822 & 401 & 0 & 4,063 & 18,871 & 971 & 59 & 0 & 43 & 212 & 35,814 \\
\hline $30-35$ & 11 & 45 & 174 & 83 & 6 & 60 & 31,154 & 69 & 21 & 91 & 2,511 & 4,430 & 198 & 0 & 20 & 169 & 39,042 \\
\hline $35-40$ & 54 & 600 & 1,997 & 504 & 1,102 & 67 & 1,990 & 7 & 14 & 17 & 92 & 11,890 & 3,421 & 1 & 0 & 610 & 22,366 \\
\hline $40-50$ & 621 & 8,011 & 142 & 56 & 29 & 918 & 190 & 2,493 & 1,258 & 163 & 28 & 5,434 & 39,247 & 779 & 239 & 4,020 & 63,628 \\
\hline Total & 2,852 & 9,135 & 9,632 & 1,114 & 2,740 & 1,818 & 40,590 & 4,811 & 1,756 & 5,893 & 23,171 & 22,873 & 42,941 & 1,510 & 3,764 & 5,404 & 180,004 \\
\hline
\end{tabular}


Table 3.16. Distribution of Low-Income Population within 80 km (50 mi) of the 200 Area Hanford Meteorological Station

\begin{tabular}{|c|c|c|c|c|c|c|c|c|c|c|c|c|c|c|c|c|c|}
\hline & \multicolumn{17}{|c|}{ Direction } \\
\hline $\begin{array}{l}\text { Distance } \\
\text { from } \\
\text { Reference } \\
\text { Point } \\
\text { (Miles) }\end{array}$ & $\mathbf{N}$ & NNE & NE & ENE & $\mathbf{E}$ & ESE & SE & SSE & $\mathbf{S}$ & SSW & SW & WSW & $\mathbf{W}$ & WNW & NW & NNW & Total \\
\hline 0-1mi & 0 & 0 & 0 & 0 & 0 & 0 & 0 & 0 & 0 & 0 & 0 & 0 & 0 & 0 & 0 & 0 & $\mathbf{0}$ \\
\hline $1-2$ & 0 & 0 & 0 & 0 & 0 & 0 & 0 & 0 & 0 & 0 & 0 & 0 & 0 & 0 & 0 & 0 & 0 \\
\hline $2-3$ & 0 & 0 & 0 & 0 & 0 & 0 & 0 & 0 & 0 & 0 & 0 & 0 & 0 & 0 & 0 & 0 & 0 \\
\hline $3-4$ & 0 & 0 & 0 & 0 & 0 & 0 & 0 & 0 & 0 & 0 & 0 & 0 & 0 & 0 & 0 & 0 & $\mathbf{0}$ \\
\hline $4-5$ & 0 & 0 & 0 & 0 & 0 & 0 & 0 & 0 & 0 & 0 & 0 & 0 & 0 & 0 & 0 & 0 & 0 \\
\hline $5-6$ & 0 & 0 & 0 & 0 & 0 & 0 & 0 & 0 & 0 & 0 & 0 & 0 & 0 & 0 & 0 & 0 & $\mathbf{0}$ \\
\hline 6-7 & 0 & 0 & 0 & 0 & 0 & 0 & 0 & 0 & 0 & 0 & 0 & 0 & 0 & 0 & 0 & 0 & $\mathbf{0}$ \\
\hline $7-8$ & 0 & 0 & 0 & 0 & 0 & 0 & 0 & 0 & 0 & 0 & 0 & 0 & 0 & 0 & 0 & 0 & $\mathbf{0}$ \\
\hline $8-9$ & 0 & 0 & 0 & 0 & 0 & 0 & 0 & 0 & 0 & 0 & 0 & 0 & 0 & 0 & 1 & 0 & 1 \\
\hline 9-10 & 0 & 0 & 0 & 0 & 0 & 0 & 0 & 0 & 0 & 0 & 0 & 0 & 1 & 0 & 2 & 1 & 4 \\
\hline 10-12 & 0 & 0 & 0 & 1 & 0 & 0 & 0 & 0 & 0 & 0 & 0 & 1 & 3 & 2 & 8 & 4 & 19 \\
\hline $12-14$ & 5 & 0 & 0 & 2 & 1 & 0 & 0 & 0 & 0 & 1 & 0 & 2 & 1 & 7 & 11 & 28 & 58 \\
\hline 14-16 & 13 & 3 & 1 & 3 & 3 & 0 & 1 & 4 & 0 & 1 & 0 & 1 & 0 & 14 & 31 & 27 & 102 \\
\hline $16-18$ & 6 & 1 & 1 & 7 & 18 & 5 & 5 & 27 & 9 & 2 & 2 & 2 & 0 & 233 & 36 & 6 & 360 \\
\hline $18-20$ & 12 & 1 & 5 & 9 & 63 & 31 & 4 & 120 & 67 & 17 & 4 & 2 & 0 & 40 & 643 & 4 & 1,022 \\
\hline 20-25 & 582 & 53 & 278 & 116 & 419 & 132 & 2,214 & 1,036 & 107 & 557 & 797 & 54 & 2 & 7 & 207 & 92 & 6,653 \\
\hline $25-30$ & 70 & 96 & 2,001 & 97 & 165 & 47 & 1,436 & 246 & 3 & 1,804 & 7,288 & 425 & 19 & 0 & 42 & 134 & 13,873 \\
\hline $30-35$ & 16 & 40 & 24 & 34 & 6 & 24 & 14,384 & 26 & 19 & 36 & 912 & 2,095 & 99 & 0 & 7 & 126 & 17,848 \\
\hline $35-40$ & 38 & 391 & 618 & 199 & 393 & 21 & 897 & 3 & 8 & 6 & 39 & 4,489 & 1,193 & 1 & 0 & 316 & 8,612 \\
\hline $40-50$ & 324 & 4,733 & 55 & 27 & 47 & 218 & 84 & 1,185 & 768 & 64 & 19 & 1,828 & 21,019 & 641 & 342 & 1,364 & 32,718 \\
\hline Total & 1,066 & 5,318 & 2,983 & 495 & 1,115 & 478 & 19,025 & 2,647 & 981 & 2,488 & 9,061 & 8,899 & 22,337 & 945 & 1,330 & 2,102 & 81,270 \\
\hline
\end{tabular}




\subsection{Area}

The 300 Area Meteorological Station is near the eastern boundary of the Hanford Site. At the 2000 Census there were about 349,100 people residing within $80 \mathrm{~km}$ (50 mi) of this location. Based on census block data, about 128,800 (37\%) of these residents were minorities. The Hispanic and Latino population (all races) was the single largest minority group, with 110,900 members (32\% of the population). There were 4,500 Native Americans (1\% of the total). There were an estimated 56,100 low-income individuals (over 16\% of the population).

Figures 3.20 to 3.25 and Tables 3.17 to 3.21 show population distributions for total, minority, and low-income populations living within 50 miles of the 300 Area Meteorological Station on the Hanford Site.

The 300 Area Meteorological Station is comparatively close to the Tri-Cities and to the relatively densely settled farmland to the east of the Columbia River. About 60,500 people live within 10 miles of the 300 Area Meteorological Station, and about 1,000 people live within 3 miles. Most of these people reside in the Richland area, ranging from SSE to SSW, but there are several hundred people living across the river within 10 miles. About 157,000 people reside within 15 miles, and 177,200 people reside within 20 miles, mostly from the SE to SW. Only 5,200 people live between 20 and 25 miles from the 300 Area Meteorological Station, mostly to the WSW. There are small concentration of Native Americans living between 5 and 20 miles to the SE and south, in the Tri-Cities area. The largest Native American population is 40-50 miles to the west, with over 1,600 individuals. Hispanic and Latino populations are located 4-8 miles due south (1,300 individuals), 10-16 miles to the SSE and SE (combined 26,700 individuals), and beyond 25 miles in several directions (mostly N-NNE, W-WSW, and due south). 


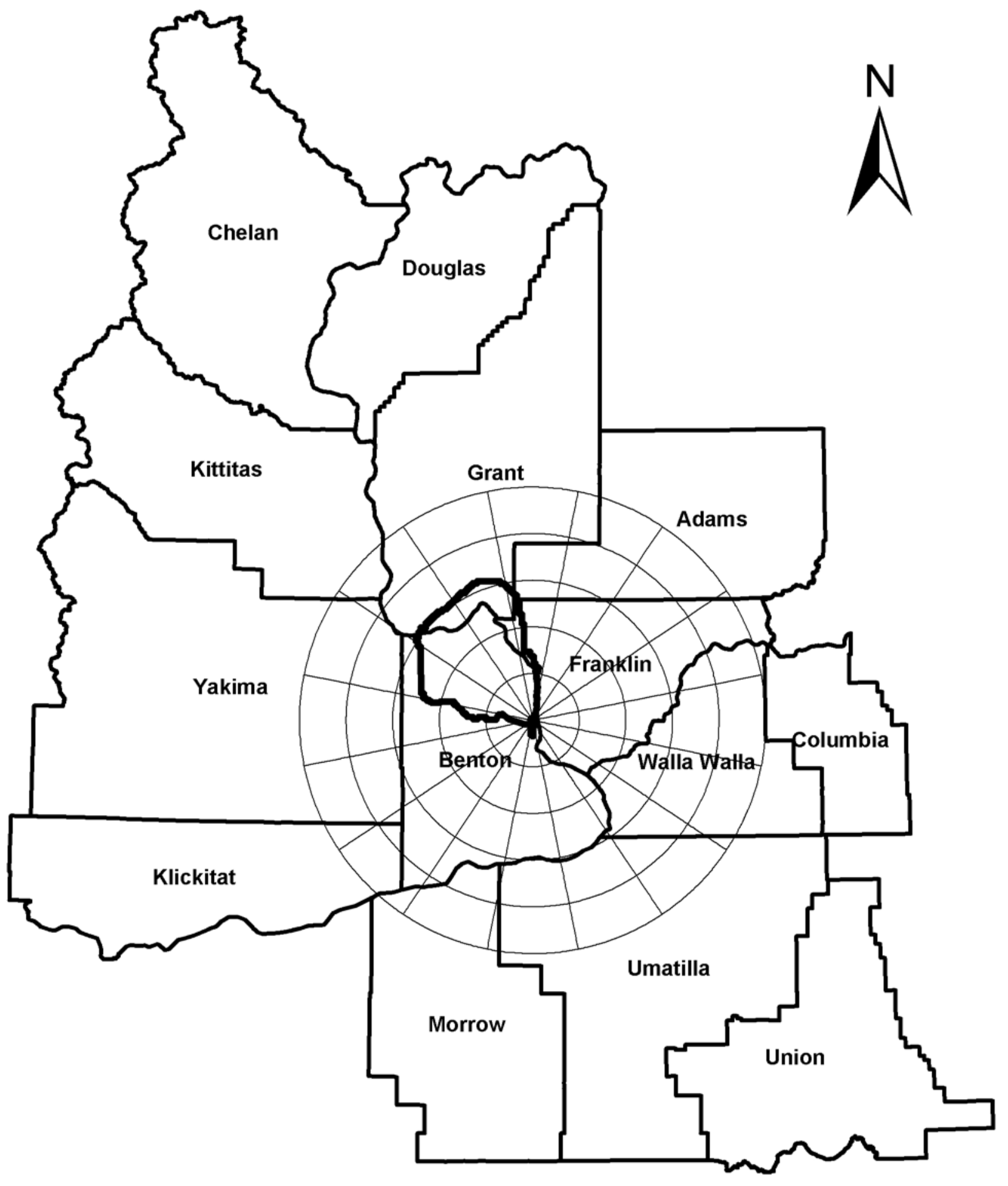

Figure 3.20. Map of $80-\mathrm{km}$ (50-mi) Region Surrounding the 300 Area Meteorological Station 


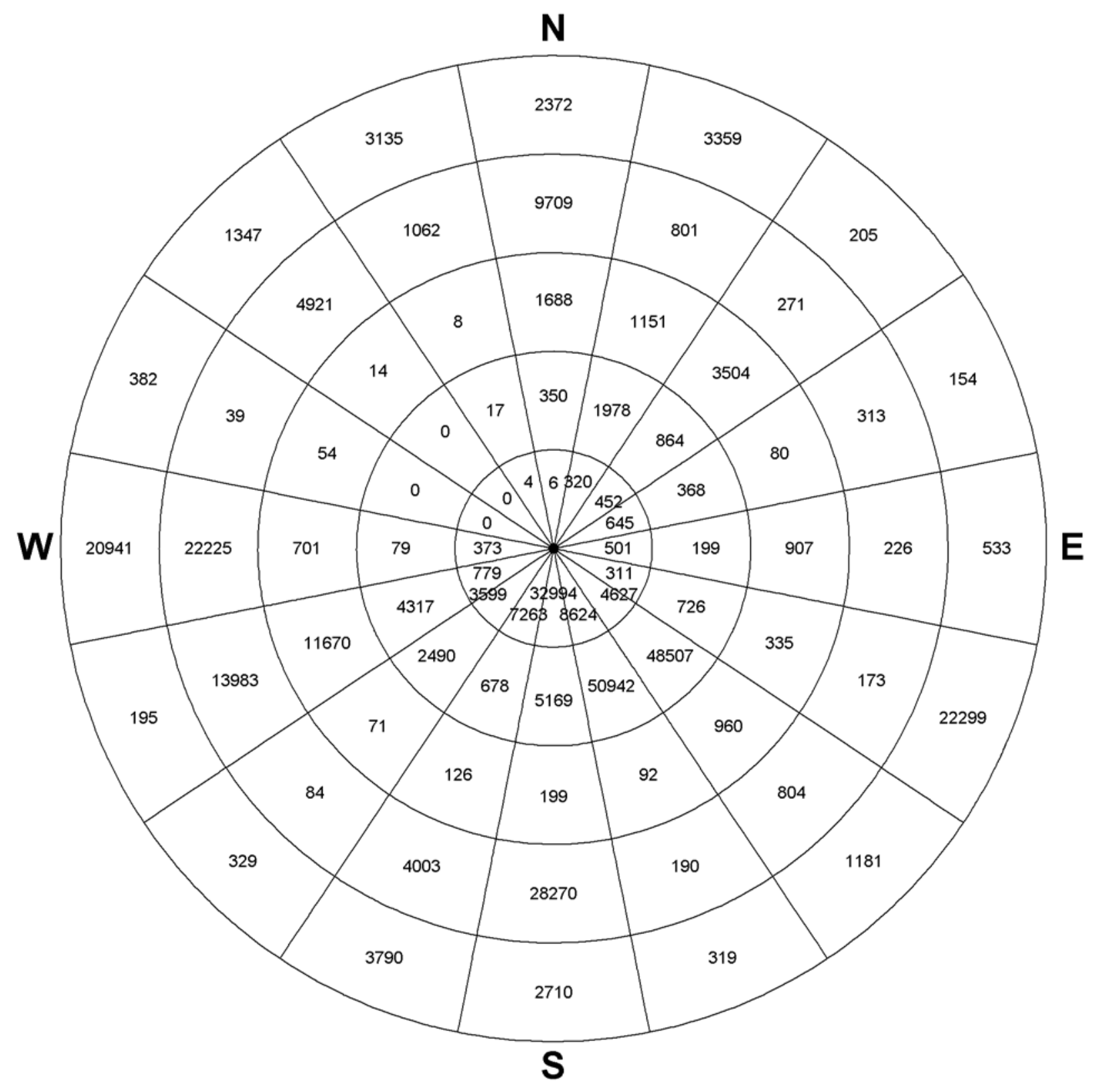

Figure 3.21. Map of Total Resident Population within $80 \mathrm{~km}(50 \mathrm{mi})$ of the Hanford 300 Area Meteorological Station, in 16-km (10-mi) Increments by Distance and Direction 


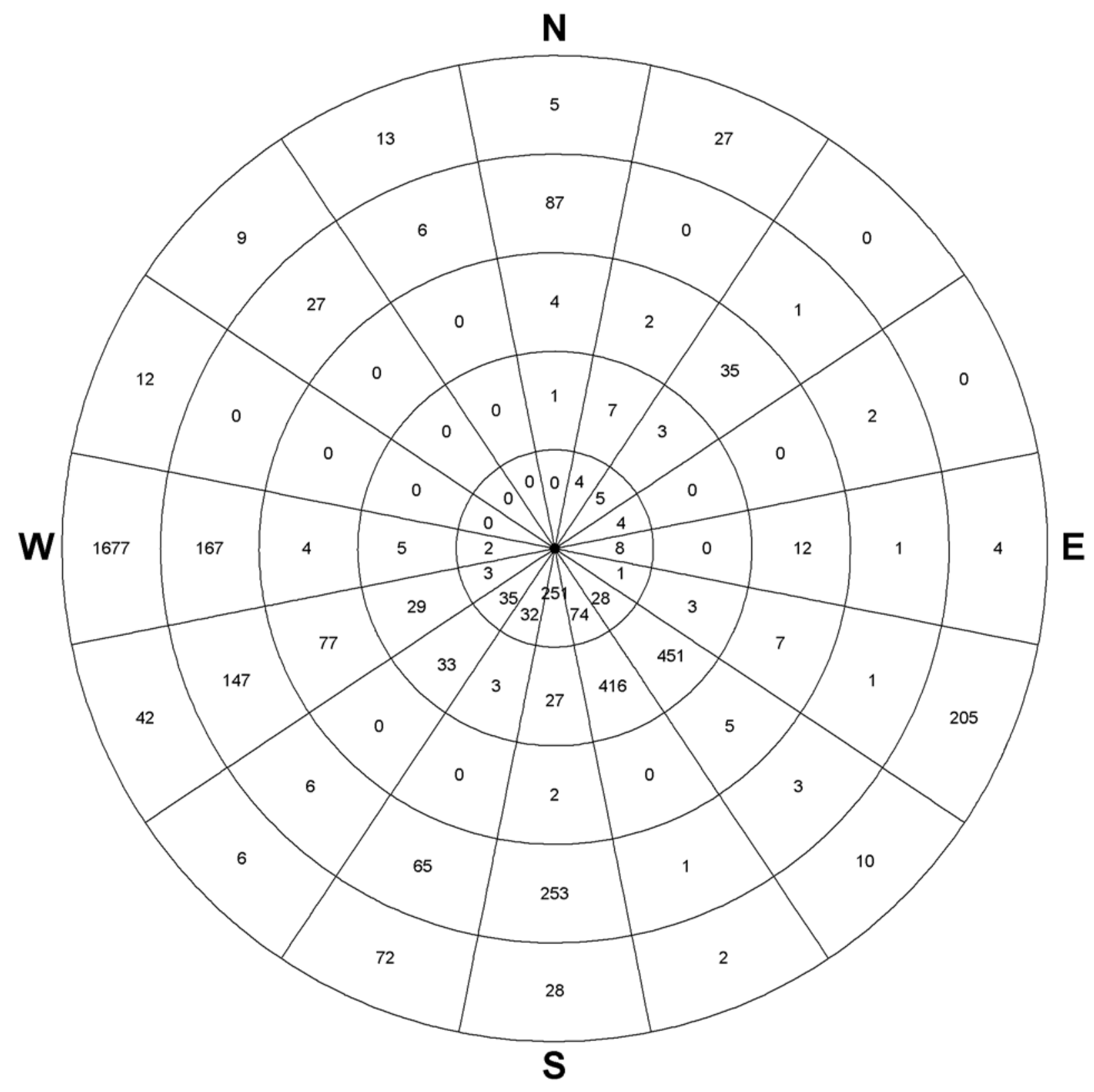

Figure 3.22. Map of Native American Population within $80 \mathrm{~km}(50 \mathrm{mi})$ of the Hanford 300 Area Meteorological Station, in 16-km (10-mi) Increments by Distance and Direction 


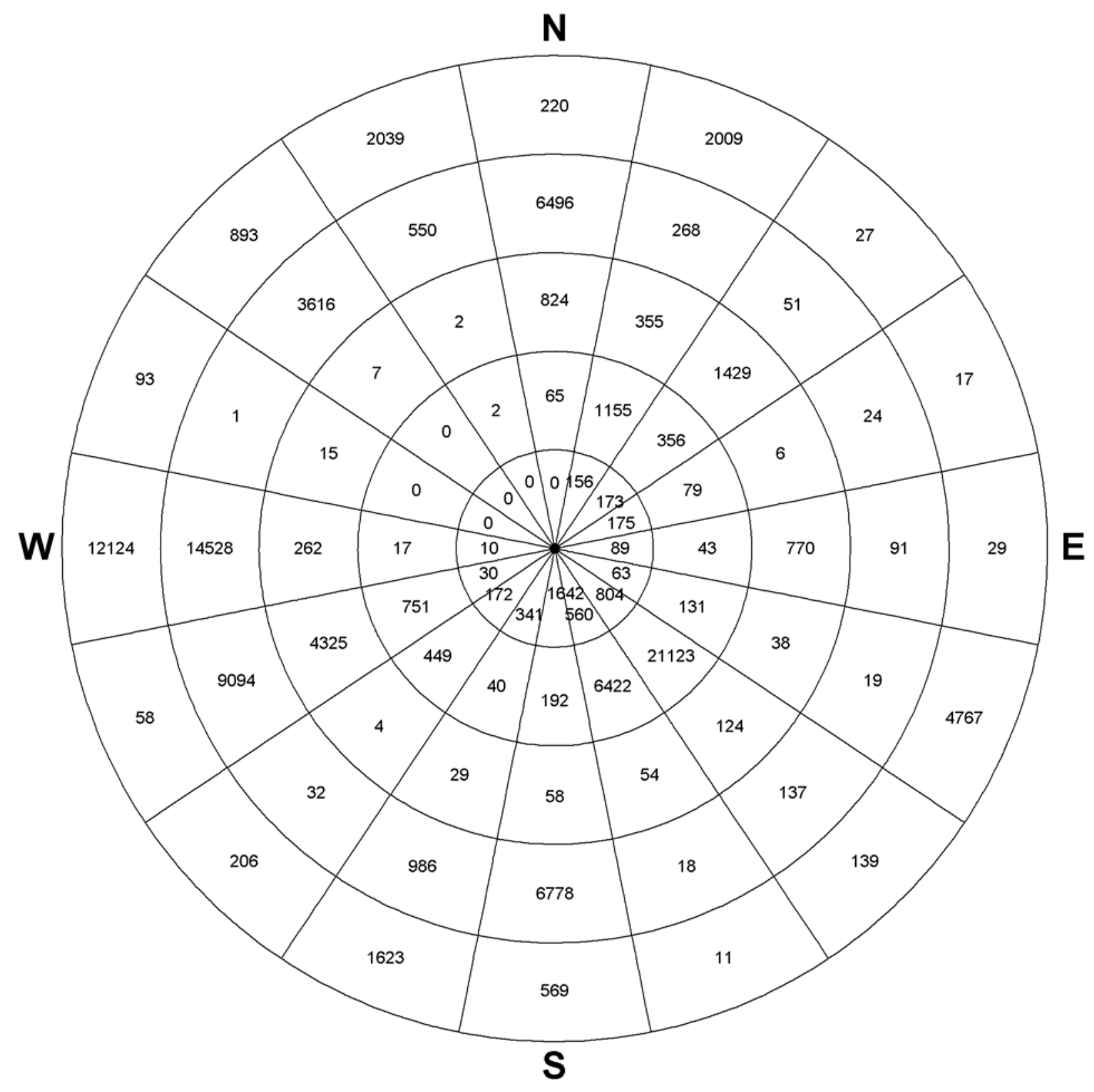

Figure 3.23. Map of Hispanic and Latino Population within $80 \mathrm{~km}(50 \mathrm{mi})$ of the Hanford 300 Area Meteorological Station, in 16-km (10-mi) Increments by Distance and Direction 


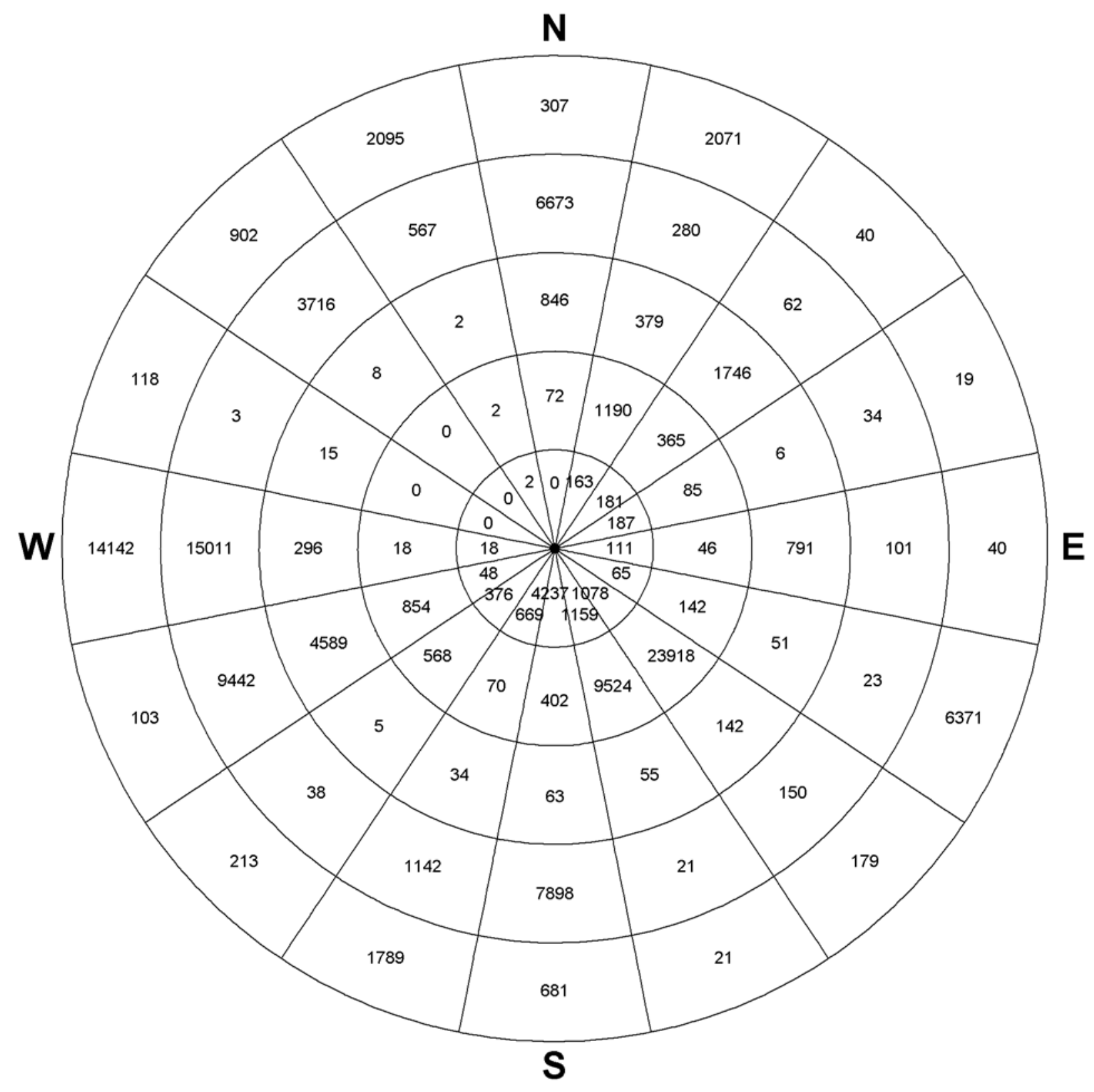

Figure 3.24. Map of Total Minority Population within $80 \mathrm{~km}(50 \mathrm{mi})$ of the Hanford 300 Area Meteorological Station, in 16-km (10-mi) Increments by Distance and Direction 


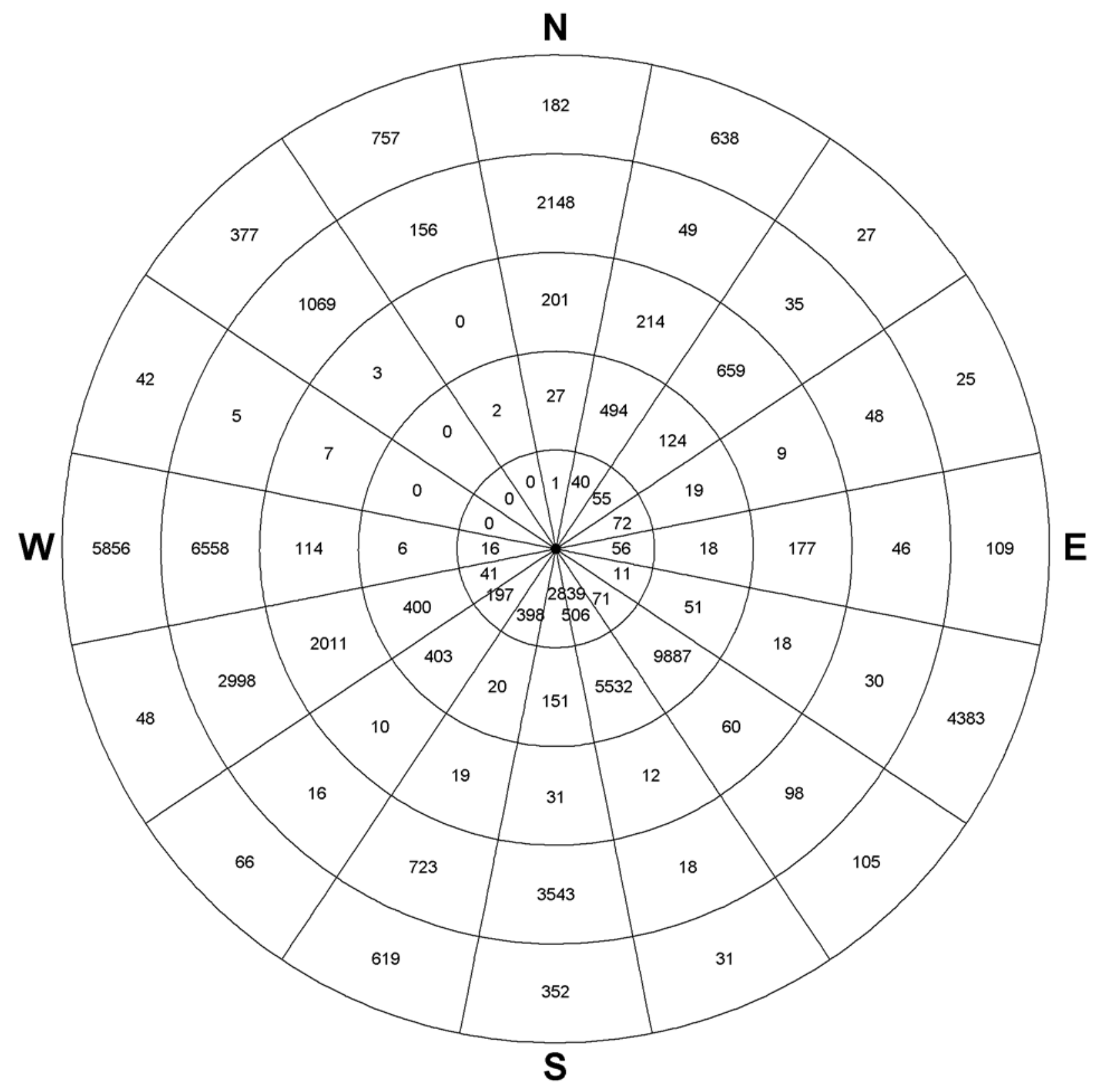

Figure 3.25. Map of Low-Income Population within $80 \mathrm{~km}(50 \mathrm{mi})$ of the Hanford 300 Area Meteorological Station, in 16-km (10-mi) Increments by Distance and Direction 
Table 3.17. Distribution of Total Resident Population within $80 \mathrm{~km}(50 \mathrm{mi})$ of the Hanford 300 Area Meteorological Station

\begin{tabular}{|c|c|c|c|c|c|c|c|c|c|c|c|c|c|c|c|c|c|}
\hline & \multicolumn{17}{|c|}{ Direction } \\
\hline $\begin{array}{l}\text { Distance } \\
\text { from } \\
\text { Reference } \\
\text { Point } \\
\text { (Miles) }\end{array}$ & $\mathbf{N}$ & NNE & NE & ENE & $\mathbf{E}$ & ESE & SE & SSE & S & SSW & SW & WSW & W & WNW & NW & NNW & Total \\
\hline 0-1mi & 0 & 0 & 0 & 0 & 0 & 0 & 0 & 0 & 0 & 0 & 0 & 0 & 0 & 0 & 0 & 0 & $\mathbf{0}$ \\
\hline 1-2 & 0 & 0 & 6 & 21 & 18 & 14 & 3 & 0 & 0 & 0 & 0 & 0 & 0 & 0 & 0 & 0 & 62 \\
\hline $2-3$ & 0 & 5 & 24 & 43 & 21 & 16 & 50 & 555 & 186 & 1 & 7 & 1 & 0 & 0 & 0 & 0 & 909 \\
\hline 3-4 & 0 & 12 & 28 & 108 & 28 & 27 & 70 & 1,841 & 2,683 & 76 & 440 & 23 & 0 & 0 & 0 & 0 & 5,336 \\
\hline $4-5$ & 0 & 21 & 64 & 59 & 132 & 49 & 39 & 331 & 8,114 & 72 & 99 & 21 & 3 & 0 & 0 & 0 & 9,004 \\
\hline 5-6 & 0 & 29 & 54 & 78 & 75 & 27 & 35 & 90 & 7,081 & 1,809 & 1,899 & 197 & 200 & 0 & 0 & 0 & 11,574 \\
\hline 6-7 & 0 & 39 & 65 & 107 & 52 & 19 & 96 & 62 & 6,202 & 3,104 & 394 & 26 & 148 & 0 & 0 & 0 & 10,314 \\
\hline $7-8$ & 0 & 47 & 42 & 65 & 122 & 19 & 322 & 813 & 1,728 & 1,929 & 283 & 52 & 7 & 0 & 0 & 4 & 5,433 \\
\hline 8-9 & 1 & 79 & 77 & 75 & 29 & 123 & 1,568 & 1,326 & 2,861 & 243 & 213 & 213 & 6 & 0 & 0 & 0 & 6,814 \\
\hline $9-10$ & 5 & 88 & 92 & 89 & 24 & 17 & 2,444 & 3,606 & 4,139 & 29 & 264 & 246 & 9 & 0 & 0 & 0 & 11,052 \\
\hline $10-12$ & 49 & 195 & 210 & 105 & 66 & 49 & 12,494 & 17,821 & 4,312 & 519 & 1,830 & 2,379 & 51 & 0 & 0 & 0 & 40,080 \\
\hline $12-14$ & 29 & 178 & 107 & 85 & 80 & 44 & 22,148 & 22,546 & 713 & 67 & 628 & 1,125 & 5 & 0 & 0 & 0 & 47,755 \\
\hline 14-16 & 52 & 403 & 190 & 124 & 21 & 95 & 5,953 & 10,076 & 130 & 50 & 5 & 277 & 1 & 0 & 0 & 1 & 17,378 \\
\hline $16-18$ & 104 & 1,088 & 117 & 36 & 22 & 350 & 4,902 & 445 & 9 & 23 & 11 & 238 & 8 & 0 & 0 & 8 & 7,361 \\
\hline $18-20$ & 116 & 114 & 240 & 18 & 10 & 188 & 3,010 & 54 & 5 & 19 & 16 & 298 & 14 & 0 & 0 & 8 & 4,110 \\
\hline $20-25$ & 169 & 330 & 319 & 31 & 49 & 331 & 706 & 20 & 6 & 85 & 41 & 3,000 & 58 & 0 & 0 & 7 & 5,152 \\
\hline $25-30$ & 1,519 & 821 & 3,185 & 49 & 858 & 4 & 254 & 72 & 193 & 41 & 30 & 8,670 & 643 & 54 & 14 & 1 & 16,408 \\
\hline $30-35$ & 9,061 & 599 & 101 & 56 & 177 & 57 & 81 & 128 & 10,513 & 3,411 & 3 & 11,142 & 12,013 & 22 & 427 & 179 & 47,970 \\
\hline $35-40$ & 648 & 202 & 170 & 257 & 49 & 116 & 723 & 62 & 17,757 & 592 & 81 & 2,841 & 10,212 & 17 & 4,494 & 883 & 39,104 \\
\hline $40-50$ & 2,372 & 3,359 & 205 & 154 & 533 & 22,299 & 1,181 & 319 & 2,710 & 3,790 & 329 & 195 & 20,941 & 382 & 1,347 & 3,135 & 63,251 \\
\hline Total & 14,125 & 7,609 & 5,296 & 1,560 & 2,366 & 23,844 & 56,079 & 60,167 & 69,342 & 15,860 & 6,573 & 30,944 & 44,319 & 475 & 6,282 & 4,226 & 349,067 \\
\hline
\end{tabular}


Table 3.18. Distribution of Native American Population within 80 km (50 mi) of the Hanford 300 Area Meteorological Station

\begin{tabular}{|c|c|c|c|c|c|c|c|c|c|c|c|c|c|c|c|c|c|}
\hline & \multicolumn{17}{|c|}{ Direction } \\
\hline $\begin{array}{l}\text { Distance } \\
\text { from } \\
\text { Reference } \\
\text { Point } \\
\text { (Miles) } \\
\end{array}$ & $\mathbf{N}$ & NNE & $\mathrm{NE}$ & ENE & $\mathbf{E}$ & ESE & SE & SSE & $\mathbf{S}$ & SSW & SW & WSW & $\mathbf{W}$ & WNW & NW & NNW & Total \\
\hline 0-1mi & 0 & 0 & 0 & 0 & 0 & 0 & 0 & 0 & 0 & 0 & 0 & 0 & 0 & 0 & 0 & 0 & 0 \\
\hline 1-2 & 0 & 0 & 0 & 0 & 0 & 0 & 0 & 0 & 0 & 0 & 0 & 0 & 0 & 0 & 0 & 0 & 0 \\
\hline $2-3$ & 0 & 0 & 0 & 0 & 0 & 0 & 0 & 15 & 0 & 0 & 0 & 0 & 0 & 0 & 0 & 0 & 15 \\
\hline 3-4 & 0 & 0 & 0 & 2 & 0 & 0 & 0 & 5 & 19 & 1 & 5 & 0 & 0 & 0 & 0 & 0 & 32 \\
\hline 4-5 & 0 & 1 & 1 & 1 & 6 & 0 & 0 & 1 & 63 & 1 & 1 & 0 & 0 & 0 & 0 & 0 & 75 \\
\hline $5-6$ & 0 & 1 & 2 & 1 & 2 & 0 & 0 & 0 & 68 & 14 & 17 & 0 & 1 & 0 & 0 & 0 & 106 \\
\hline 6-7 & 0 & 1 & 2 & 0 & 0 & 0 & 1 & 0 & 63 & 11 & 3 & 0 & 1 & 0 & 0 & 0 & 82 \\
\hline $7-8$ & 0 & 1 & 0 & 0 & 0 & 0 & 4 & 3 & 6 & 4 & 3 & 0 & 0 & 0 & 0 & 0 & 21 \\
\hline 8-9 & 0 & 0 & 0 & 0 & 0 & 1 & 13 & 11 & 8 & 1 & 1 & 2 & 0 & 0 & 0 & 0 & 37 \\
\hline $9-10$ & 0 & 0 & 0 & 0 & 0 & 0 & 10 & 39 & 24 & 0 & 5 & 1 & 0 & 0 & 0 & 0 & 79 \\
\hline $10-12$ & 1 & 0 & 0 & 0 & 0 & 0 & 94 & 124 & 15 & 3 & 23 & 12 & 0 & 0 & 0 & 0 & 272 \\
\hline $12-14$ & 0 & 1 & 1 & 0 & 0 & 0 & 194 & 221 & 8 & 0 & 10 & 13 & 0 & 0 & 0 & 0 & 448 \\
\hline 14-16 & 0 & 0 & 0 & 0 & 0 & 0 & 95 & 69 & 4 & 0 & 0 & 1 & 0 & 0 & 0 & 0 & 169 \\
\hline $16-18$ & 0 & 6 & 0 & 0 & 0 & 2 & 45 & 2 & 0 & 0 & 0 & 3 & 5 & 0 & 0 & 0 & 63 \\
\hline $18-20$ & 0 & 0 & 2 & 0 & 0 & 1 & 23 & 0 & 0 & 0 & 0 & 0 & 0 & 0 & 0 & 0 & 26 \\
\hline 20-25 & 0 & 1 & 2 & 0 & 0 & 5 & 3 & 0 & 0 & 0 & 0 & 12 & 0 & 0 & 0 & 0 & 23 \\
\hline $25-30$ & 4 & 1 & 33 & 0 & 12 & 2 & 2 & 0 & 2 & 0 & 0 & 65 & 4 & 0 & 0 & 0 & 125 \\
\hline 30-35 & 83 & 0 & 0 & 0 & 1 & 1 & 0 & 1 & 99 & 58 & 0 & 100 & 103 & 0 & 5 & 0 & 451 \\
\hline $35-40$ & 4 & 0 & 1 & 2 & 0 & 0 & 3 & 0 & 154 & 7 & 6 & 47 & 64 & 0 & 22 & 6 & 316 \\
\hline $40-50$ & 5 & 27 & 0 & 0 & 4 & 205 & 10 & 2 & 28 & 72 & 6 & 42 & 1,677 & 12 & 9 & 13 & 2,112 \\
\hline Total & 97 & 40 & 44 & 6 & 25 & 217 & 497 & 493 & 561 & 172 & 80 & 298 & 1,855 & 12 & 36 & 19 & 4,452 \\
\hline
\end{tabular}


Table 3.19. Distribution of Hispanic and Latino Population within 80 km (50 mi) of the Hanford 300 Area Meteorological Station

\begin{tabular}{|c|c|c|c|c|c|c|c|c|c|c|c|c|c|c|c|c|c|}
\hline & \multicolumn{17}{|c|}{ Direction } \\
\hline $\begin{array}{l}\text { Distance } \\
\text { from } \\
\text { Reference } \\
\text { Point } \\
\text { (Miles) }\end{array}$ & $\mathbf{N}$ & NNE & NE & ENE & $\mathbf{E}$ & ESE & SE & SSE & $\mathbf{S}$ & SSW & SW & WSW & W & WNW & NW & NNW & Total \\
\hline 0-1mi & 0 & 0 & 0 & 0 & 0 & 0 & 0 & 0 & 0 & 0 & 0 & 0 & 0 & 0 & 0 & 0 & $\mathbf{0}$ \\
\hline 1-2 & 0 & 0 & 2 & 0 & 0 & 0 & 0 & 0 & 0 & 0 & 0 & 0 & 0 & 0 & 0 & 0 & 2 \\
\hline $2-3$ & 0 & 2 & 4 & 15 & 0 & 0 & 4 & 27 & 5 & 0 & 0 & 0 & 0 & 0 & 0 & 0 & 57 \\
\hline 3-4 & 0 & 6 & 4 & 11 & 1 & 0 & 10 & 35 & 85 & 3 & 14 & 1 & 0 & 0 & 0 & 0 & 170 \\
\hline $4-5$ & 0 & 10 & 25 & 7 & 16 & 2 & 2 & 8 & 470 & 3 & 1 & 0 & 0 & 0 & 0 & 0 & 544 \\
\hline 5-6 & 0 & 14 & 26 & 19 & 17 & 2 & 2 & 3 & 364 & 96 & 81 & 7 & 2 & 0 & 0 & 0 & 633 \\
\hline 6-7 & 0 & 20 & 30 & 42 & 15 & 3 & 28 & 5 & 286 & 139 & 35 & 3 & 3 & 0 & 0 & 0 & 609 \\
\hline $7-8$ & 0 & 25 & 8 & 13 & 32 & 4 & 90 & 45 & 133 & 91 & 14 & 8 & 0 & 0 & 0 & 0 & 463 \\
\hline 8-9 & 0 & 39 & 34 & 20 & 5 & 47 & 303 & 92 & 97 & 8 & 11 & 8 & 2 & 0 & 0 & 0 & 666 \\
\hline $9-10$ & 0 & 40 & 40 & 48 & 3 & 5 & 365 & 345 & 202 & 1 & 16 & 3 & 3 & 0 & 0 & 0 & 1,071 \\
\hline $10-12$ & 11 & 93 & 110 & 30 & 7 & 16 & 5,175 & 1,874 & 144 & 24 & 301 & 310 & 5 & 0 & 0 & 0 & 8,100 \\
\hline $12-14$ & 6 & 68 & 27 & 5 & 8 & 16 & 13,706 & 3,935 & 40 & 7 & 148 & 159 & 3 & 0 & 0 & 0 & 18,128 \\
\hline 14-16 & 5 & 217 & 79 & 33 & 10 & 26 & 1,462 & 570 & 3 & 5 & 0 & 60 & 0 & 0 & 0 & 0 & 2,470 \\
\hline $16-18$ & 16 & 756 & 29 & 8 & 12 & 50 & 492 & 40 & 2 & 3 & 0 & 96 & 5 & 0 & 0 & 1 & 1,510 \\
\hline $18-20$ & 27 & 21 & 111 & 3 & 6 & 23 & 288 & 3 & 3 & 1 & 0 & 126 & 4 & 0 & 0 & 1 & 617 \\
\hline $20-25$ & 52 & 108 & 164 & 3 & 10 & 38 & 76 & 6 & 1 & 15 & 2 & 1,195 & 26 & 0 & 0 & 1 & 1,697 \\
\hline $25-30$ & 772 & 247 & 1,265 & 3 & 760 & 0 & 48 & 48 & 57 & 14 & 2 & 3,130 & 236 & 15 & 7 & 1 & 6,605 \\
\hline $30-35$ & 6,181 & 197 & 21 & 4 & 88 & 8 & 16 & 16 & 2,776 & 829 & 1 & 6,836 & 8,555 & 0 & 236 & 114 & 25,878 \\
\hline $35-40$ & 315 & 71 & 30 & 20 & 3 & 11 & 121 & 2 & 4,002 & 157 & 31 & 2,258 & 5,973 & 1 & 3,380 & 436 & 16,811 \\
\hline $40-50$ & 220 & 2,009 & 27 & 17 & 29 & 4,767 & 139 & 11 & 569 & 1,623 & 206 & 58 & 12,124 & 93 & 893 & 2,039 & 24,824 \\
\hline Total & 7,605 & 3,943 & 2,036 & 301 & 1,022 & 5,018 & 22,327 & 7,065 & 9,239 & 3,019 & 863 & 14,258 & 26,941 & 109 & 4,516 & 2,593 & 110,855 \\
\hline
\end{tabular}


Table 3.20. Distribution of Total Minority Population within $80 \mathrm{~km}(50 \mathrm{mi})$ of the Hanford 300 Area Meteorological Station

\begin{tabular}{|c|c|c|c|c|c|c|c|c|c|c|c|c|c|c|c|c|c|}
\hline & \multicolumn{17}{|c|}{ Direction } \\
\hline $\begin{array}{l}\text { Distance } \\
\text { from } \\
\text { Reference } \\
\text { Point } \\
\text { (Miles) } \\
\end{array}$ & $\mathbf{N}$ & NNE & NE & ENE & $\mathbf{E}$ & ESE & SE & SSE & 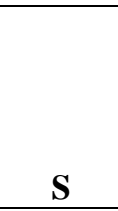 & SSW & SW & WSW & $\mathbf{W}$ & WNW & NW & NNW & Total \\
\hline 0-1mi & 0 & 0 & 0 & 0 & 0 & 0 & 0 & 0 & 0 & 0 & 0 & 0 & 0 & 0 & 0 & 0 & $\mathbf{0}$ \\
\hline 1-2 & 0 & 0 & 2 & 1 & 1 & 0 & 0 & 0 & 0 & 0 & 0 & 0 & 0 & 0 & 0 & 0 & 4 \\
\hline $2-3$ & 0 & 2 & 6 & 16 & 0 & 0 & 4 & 103 & 32 & 0 & 2 & 0 & 0 & 0 & 0 & 0 & 165 \\
\hline $3-4$ & 0 & 7 & 4 & 13 & 1 & 0 & 10 & 220 & 344 & 16 & 75 & 5 & 0 & 0 & 0 & 0 & 695 \\
\hline $4-5$ & 0 & 11 & 26 & 8 & 25 & 2 & 4 & 28 & 1,239 & 6 & 7 & 1 & 0 & 0 & 0 & 0 & 1,357 \\
\hline $5-6$ & 0 & 15 & 27 & 21 & 21 & 3 & 3 & 5 & 785 & 178 & 165 & 11 & 6 & 0 & 0 & 0 & 1,240 \\
\hline 6-7 & 0 & 22 & 31 & 45 & 16 & 3 & 29 & 9 & 691 & 270 & 58 & 4 & 6 & 0 & 0 & 0 & 1,184 \\
\hline $7-8$ & 0 & 27 & 10 & 13 & 34 & 4 & 100 & 81 & 304 & 177 & 19 & 8 & 1 & 0 & 0 & 2 & 780 \\
\hline $8-9$ & 0 & 39 & 34 & 20 & 8 & 48 & 440 & 149 & 320 & 21 & 15 & 13 & 2 & 0 & 0 & 0 & 1,109 \\
\hline 9-10 & 0 & 40 & 41 & 50 & 5 & 5 & 488 & 564 & 522 & 1 & 35 & 6 & 3 & 0 & 0 & 0 & 1,760 \\
\hline 10-12 & 13 & 93 & 112 & 31 & 9 & 16 & 6,104 & 3,032 & 326 & 49 & 399 & 355 & 6 & 0 & 0 & 0 & 10,545 \\
\hline $12-14$ & 7 & 72 & 29 & 6 & 9 & 16 & 15,012 & 5,394 & 62 & 9 & 169 & 204 & 3 & 0 & 0 & 0 & 20,992 \\
\hline 14-16 & 6 & 224 & 79 & 37 & 10 & 27 & 1,768 & 1,046 & 9 & 6 & 0 & 61 & 0 & 0 & 0 & 0 & 3,273 \\
\hline 16-18 & 17 & 776 & 29 & 8 & 12 & 57 & 667 & 49 & 2 & 4 & 0 & 101 & 5 & 0 & 0 & 1 & 1,728 \\
\hline $18-20$ & 29 & 25 & 116 & 3 & 6 & 26 & 367 & 3 & 3 & 2 & 0 & 133 & 4 & 0 & 0 & 1 & 718 \\
\hline 20-25 & 55 & 114 & 172 & 3 & 14 & 49 & 92 & 6 & 1 & 19 & 3 & 1,266 & 28 & 0 & 0 & 1 & 1,823 \\
\hline $25-30$ & 791 & 265 & 1,574 & 3 & 777 & 2 & 50 & 49 & 62 & 15 & 2 & 3,323 & 268 & 15 & 8 & 1 & 7,205 \\
\hline 30-35 & 6,336 & 208 & 24 & 4 & 98 & 12 & 16 & 17 & 3,164 & 963 & 1 & 7,087 & 8,792 & 1 & 256 & 115 & 27,094 \\
\hline $35-40$ & 337 & 72 & 38 & 30 & 3 & 11 & 134 & 4 & 4,734 & 179 & 37 & 2,355 & 6,219 & 2 & 3,460 & 452 & 18,067 \\
\hline $40-50$ & 307 & 2,071 & 40 & 19 & 40 & 6,371 & 179 & 21 & 681 & 1,789 & 213 & 103 & 14,142 & 118 & 902 & 2,095 & 29,091 \\
\hline Total & 7,898 & 4,083 & 2,394 & 331 & 1,089 & 6,652 & 25,467 & 10,780 & 13,281 & 3,704 & 1,200 & 15,036 & 29,485 & 136 & 4,626 & 2,668 & 128,830 \\
\hline
\end{tabular}


Table 3.21. Distribution of Low-Income Population within 80 km (50 mi) of the Hanford 300 Area Meteorological Station

\begin{tabular}{|c|c|c|c|c|c|c|c|c|c|c|c|c|c|c|c|c|c|}
\hline & \multicolumn{17}{|c|}{ Direction } \\
\hline $\begin{array}{l}\text { Distance } \\
\text { from } \\
\text { Reference } \\
\text { Point } \\
\text { (Miles) } \\
\end{array}$ & $\mathbf{N}$ & NNE & NE & ENE & $\mathbf{E}$ & ESE & SE & SSE & $\mathbf{S}$ & SSW & SW & WSW & $\mathbf{W}$ & WNW & NW & NNW & Total \\
\hline 0-1mi & 0 & 0 & 0 & 0 & 0 & 0 & 0 & 0 & 0 & 0 & 0 & 0 & 0 & 0 & 0 & 0 & 0 \\
\hline 1-2 & 0 & 0 & 1 & 3 & 2 & 0 & 0 & 0 & 0 & 0 & 0 & 0 & 0 & 0 & 0 & 0 & 6 \\
\hline $2-3$ & 0 & 1 & 3 & 5 & 2 & 0 & 0 & 10 & 0 & 0 & 0 & 0 & 0 & 0 & 0 & 0 & 21 \\
\hline $3-4$ & 0 & 1 & 3 & 13 & 3 & 1 & 0 & 48 & 172 & 3 & 19 & 1 & 0 & 0 & 0 & 0 & 264 \\
\hline $4-5$ & 0 & 3 & 8 & 7 & 16 & 4 & 0 & 17 & 979 & 4 & 7 & 1 & 0 & 0 & 0 & 0 & 1,046 \\
\hline $5-6$ & 0 & 3 & 7 & 9 & 9 & 2 & 0 & 0 & 589 & 112 & 105 & 9 & 9 & 0 & 0 & 0 & 854 \\
\hline 6-7 & 0 & 5 & 8 & 13 & 6 & 1 & 0 & 0 & 652 & 160 & 21 & 1 & 7 & 0 & 0 & 0 & 874 \\
\hline $7-8$ & 0 & 6 & 5 & 8 & 15 & 1 & 2 & 14 & 150 & 97 & 17 & 3 & 0 & 0 & 0 & 0 & 318 \\
\hline $8-9$ & 0 & 10 & 9 & 8 & 2 & 1 & 21 & 103 & 77 & 21 & 12 & 12 & 0 & 0 & 0 & 0 & 276 \\
\hline 9-10 & 1 & 11 & 11 & 6 & 1 & 1 & 48 & 314 & 220 & 1 & 16 & 14 & 0 & 0 & 0 & 0 & 644 \\
\hline 10-12 & 6 & 23 & 19 & 4 & 6 & 5 & 2,409 & 1,733 & 126 & 14 & 278 & 198 & 3 & 0 & 0 & 0 & 4,824 \\
\hline $12-14$ & 3 & 29 & 7 & 4 & 7 & 6 & 5,915 & 3,403 & 19 & 2 & 123 & 112 & 0 & 0 & 0 & 0 & 9,630 \\
\hline 14-16 & 3 & 107 & 9 & 6 & 2 & 9 & 1,027 & 353 & 4 & 1 & 0 & 22 & 0 & 0 & 0 & 0 & 1,543 \\
\hline 16-18 & 7 & 312 & 22 & 3 & 2 & 19 & 323 & 38 & 1 & 1 & 1 & 25 & 1 & 0 & 0 & 1 & 756 \\
\hline $18-20$ & 8 & 23 & 67 & 2 & 1 & 12 & 213 & 5 & 1 & 2 & 1 & 43 & 2 & 0 & 0 & 1 & 381 \\
\hline 20-25 & 11 & 89 & 73 & 3 & 4 & 17 & 29 & 2 & 1 & 13 & 6 & 632 & 7 & 0 & 0 & 0 & 887 \\
\hline 25-30 & 190 & 125 & 586 & 6 & 173 & 1 & 31 & 10 & 30 & 6 & 4 & 1,379 & 107 & 7 & 3 & 0 & 2,658 \\
\hline 30-35 & 2,050 & 42 & 12 & 8 & 36 & 10 & 10 & 14 & 1,537 & 643 & 0 & 2,191 & 3,963 & 3 & 93 & 37 & 10,649 \\
\hline $35-40$ & 98 & 7 & 23 & 40 & 10 & 20 & 88 & 4 & 2,006 & 80 & 16 & 807 & 2,595 & 2 & 976 & 119 & 6,891 \\
\hline $40-50$ & 182 & 638 & 27 & 25 & 109 & 4,383 & 105 & 31 & 352 & 619 & 66 & 48 & 5,856 & 42 & 377 & 757 & 13,617 \\
\hline Total & 2,559 & 1,435 & 900 & 173 & 406 & 4,493 & 10,221 & 6,099 & 6,916 & 1,779 & 692 & 5,498 & 12,550 & 54 & 1,449 & 915 & 56,139 \\
\hline
\end{tabular}




\subsection{Area}

The 400 Area Meteorological Station is in the south central part of the Hanford Site. At the 2000 Census there were about 353,700 people residing within $80 \mathrm{~km}$ (50 mi) of this location. Based on census block data, about 131,700 (37\%) of these residents were minorities. The Hispanic and Latino population (all races) was the single largest minority group, with 113,100 members (32\% of the population). There were 5,300 Native Americans (1\% of the total). There were an estimated 55,700 low-income individuals (about $16 \%$ of the population).

Figures 3.26 to 3.31 and Tables 3.22 to 3.26 show population distributions for total, minority, and low-income populations living within 50 miles of the 400 Area Meteorological Station on the Hanford Site.

The 400 Area Meteorological Station is comparatively close to the Tri-Cities, but a few miles further away than the 300 Area from both the city of Richland to the south and the settled farmland to the east of the Columbia River. About 19,700 people live within 10 miles of the 400 Area Meteorological Station, but only about 100 people live within 5 miles, and there are no residents within 3 miles. Most of these people reside in the Richland area, ranging from SSE to SSW, but there are few hundred people living within 10 miles across the river toward the east. About 65,500 people reside within 15 miles, and 156,600 people reside within 20 miles, mostly from the SE to SW. There are small concentrations of Native Americans living between 8 and 25 miles to the SE and south, in the Tri-Cities area. The largest Native American population is 40-50 miles to the west, with about 2,400 individuals. Hispanic and Latino populations are located 8 to 20 miles SE to due south (over 29,000 individuals), 25-35 miles to the NW through NE and WSW, and beyond 35 miles in several directions (mostly N-NNE, west, and south). 


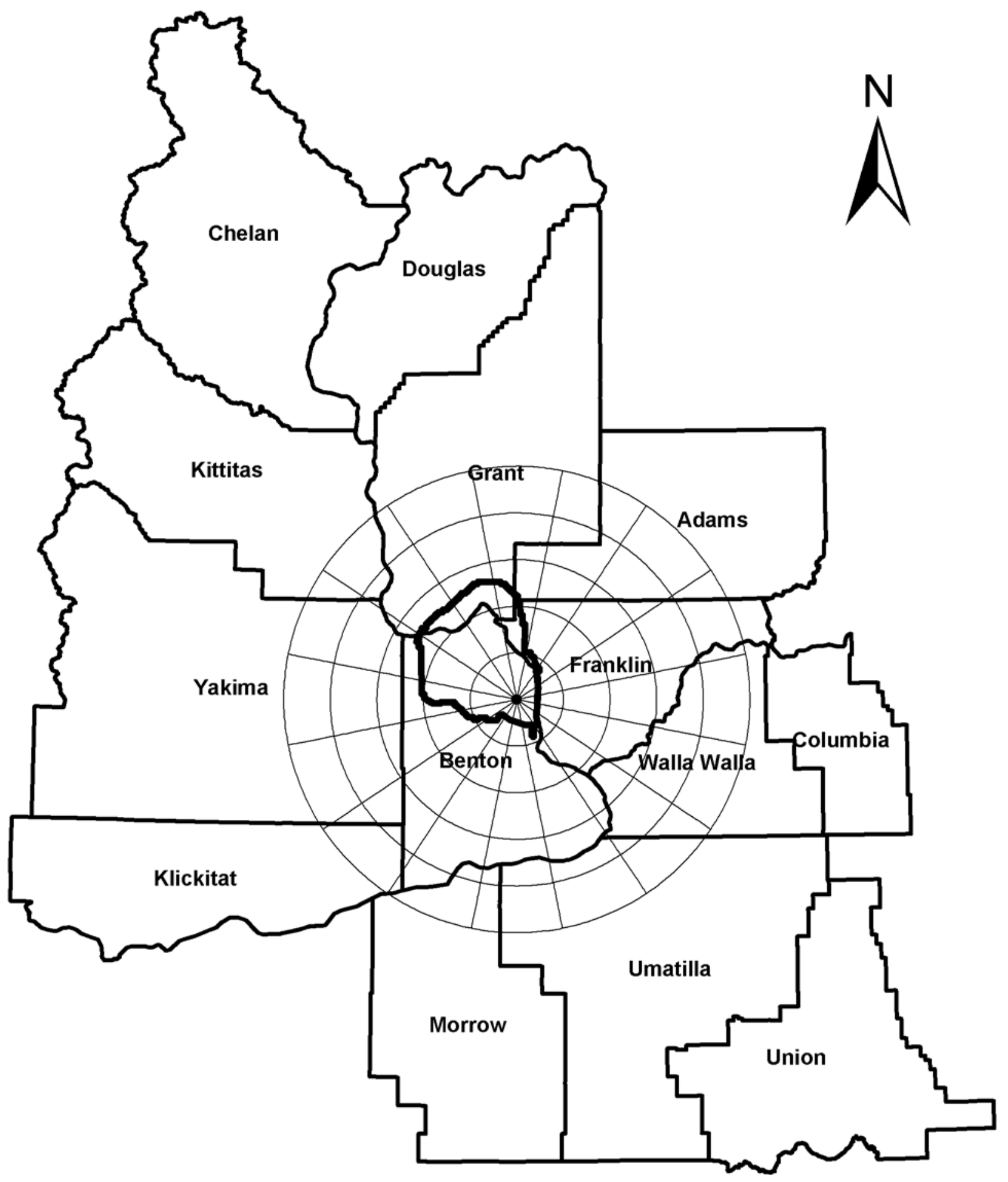

Figure 3.26. Map of $80-\mathrm{km}$ (50-mi) Region Surrounding the 400 Area Meteorological Station 


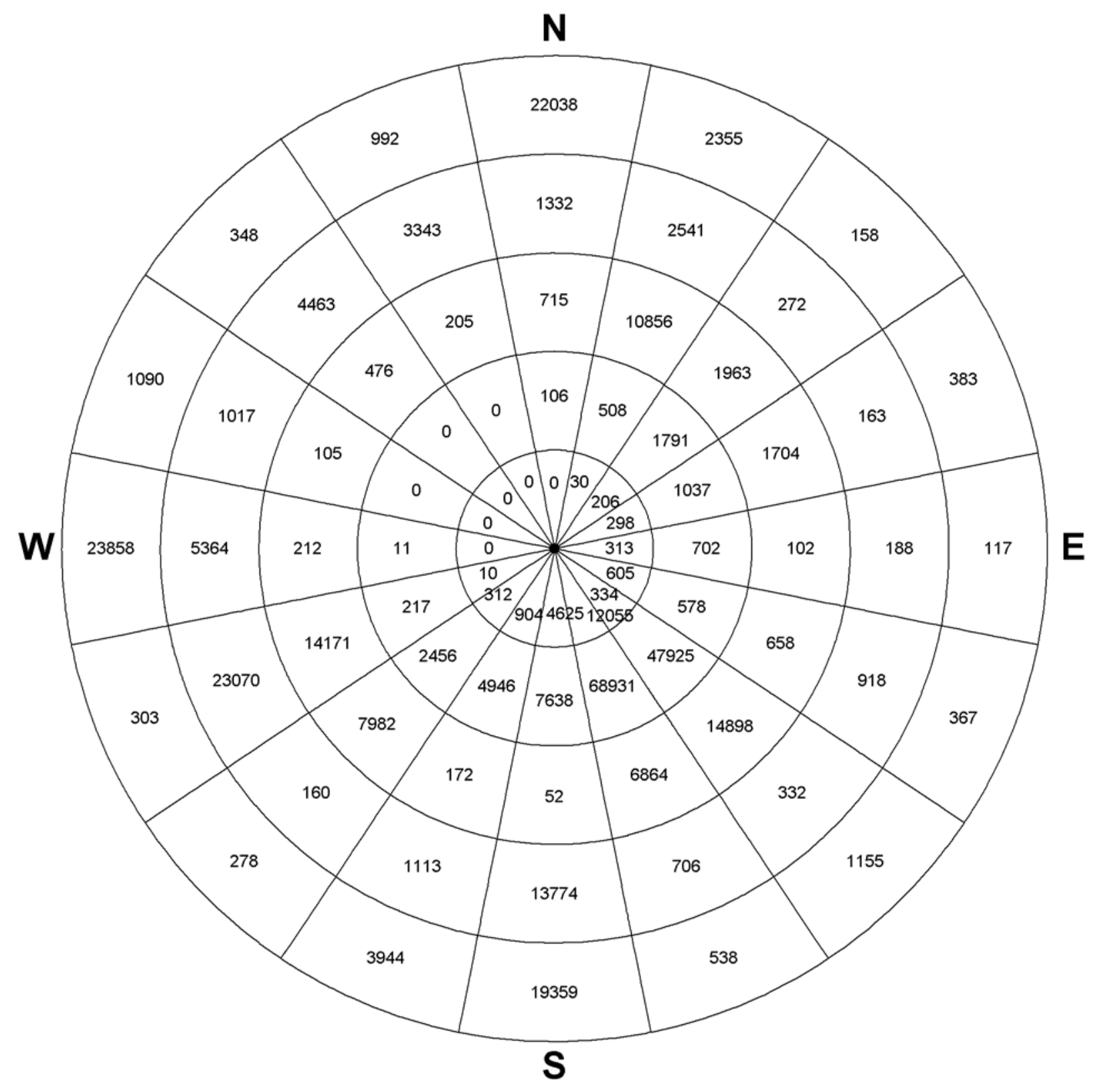

Figure 3.27. Map of Total Resident Population within $80 \mathrm{~km}(50 \mathrm{mi})$ of the Hanford 400 Area Meteorological Station, in 16-km (10-mi) Increments by Distance and Direction 


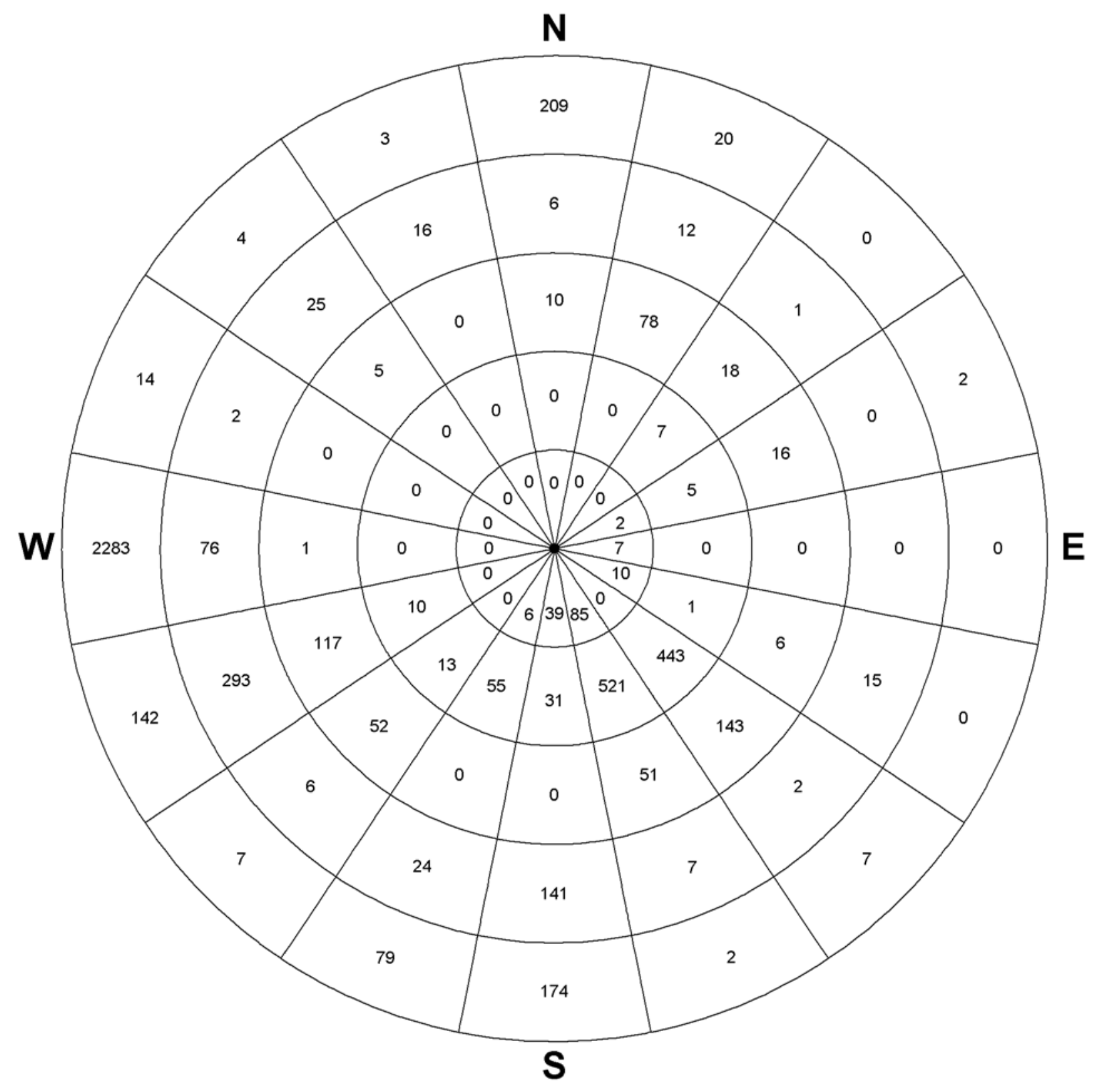

Figure 3.28. Map of Native American Population within $80 \mathrm{~km}(50 \mathrm{mi})$ of the Hanford 400 Area Meteorological Station, in 16-km (10-mi) Increments by Distance and Direction 


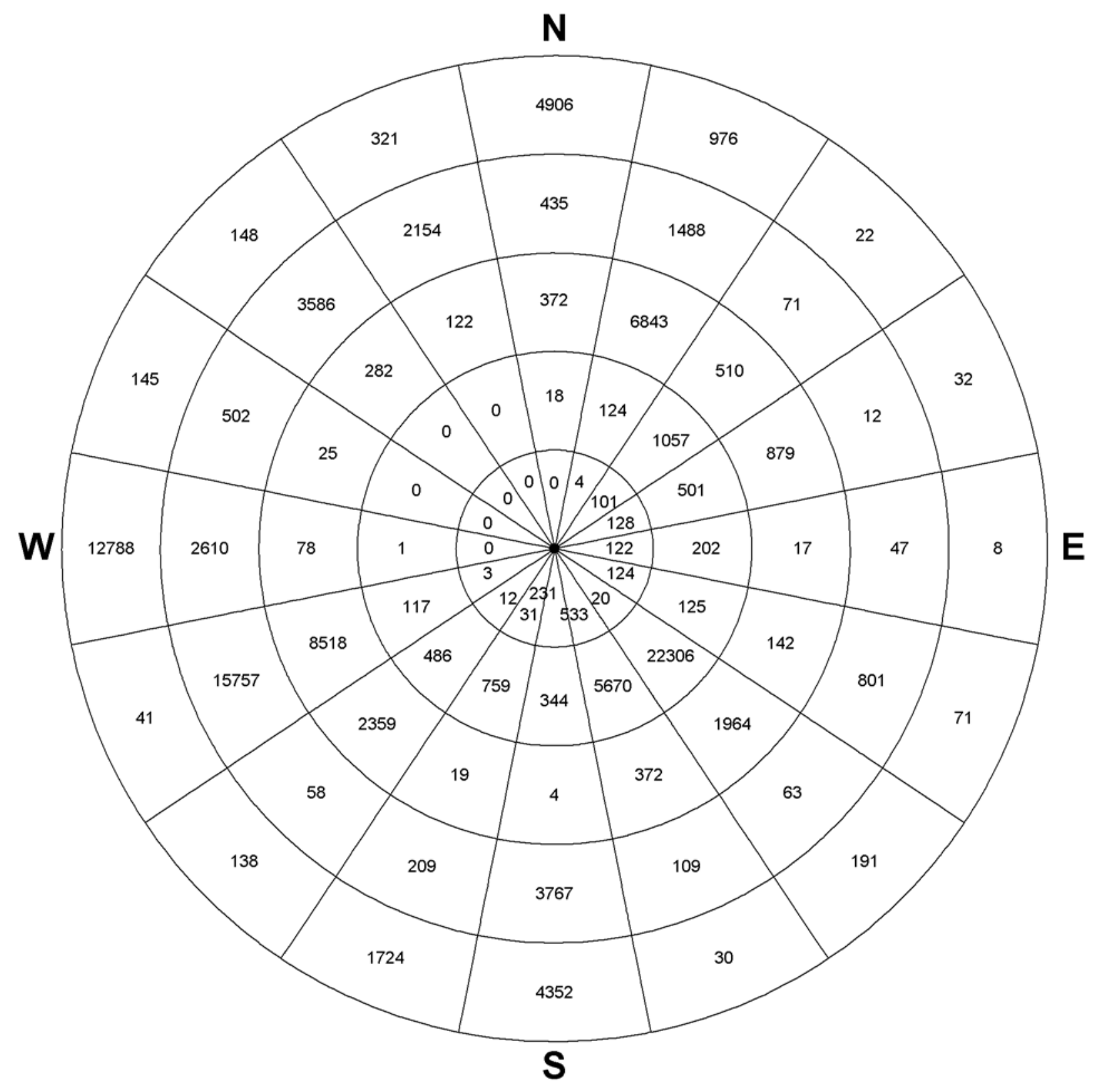

Figure 3.29. Map of Hispanic and Latino Population within $80 \mathrm{~km}(50 \mathrm{mi})$ of the Hanford 400 Area Meteorological Station, in 16-km (10-mi) Increments by Distance and Direction 


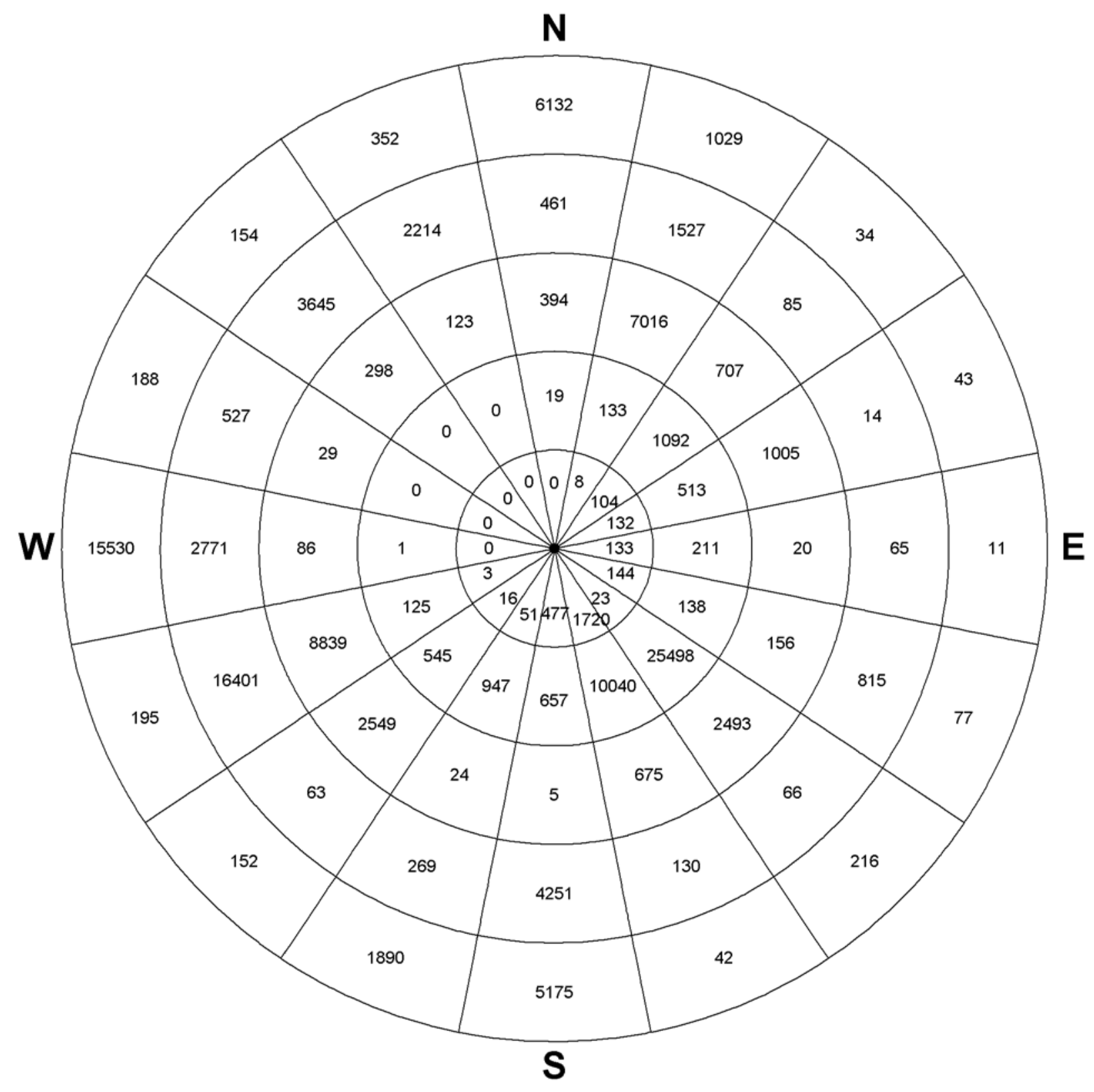

Figure 3.30. Map of Total Minority Population within $80 \mathrm{~km}(50 \mathrm{mi})$ of the Hanford 400 Area Meteorological Station, in 16-km (10-mi) Increments by Distance and Direction 


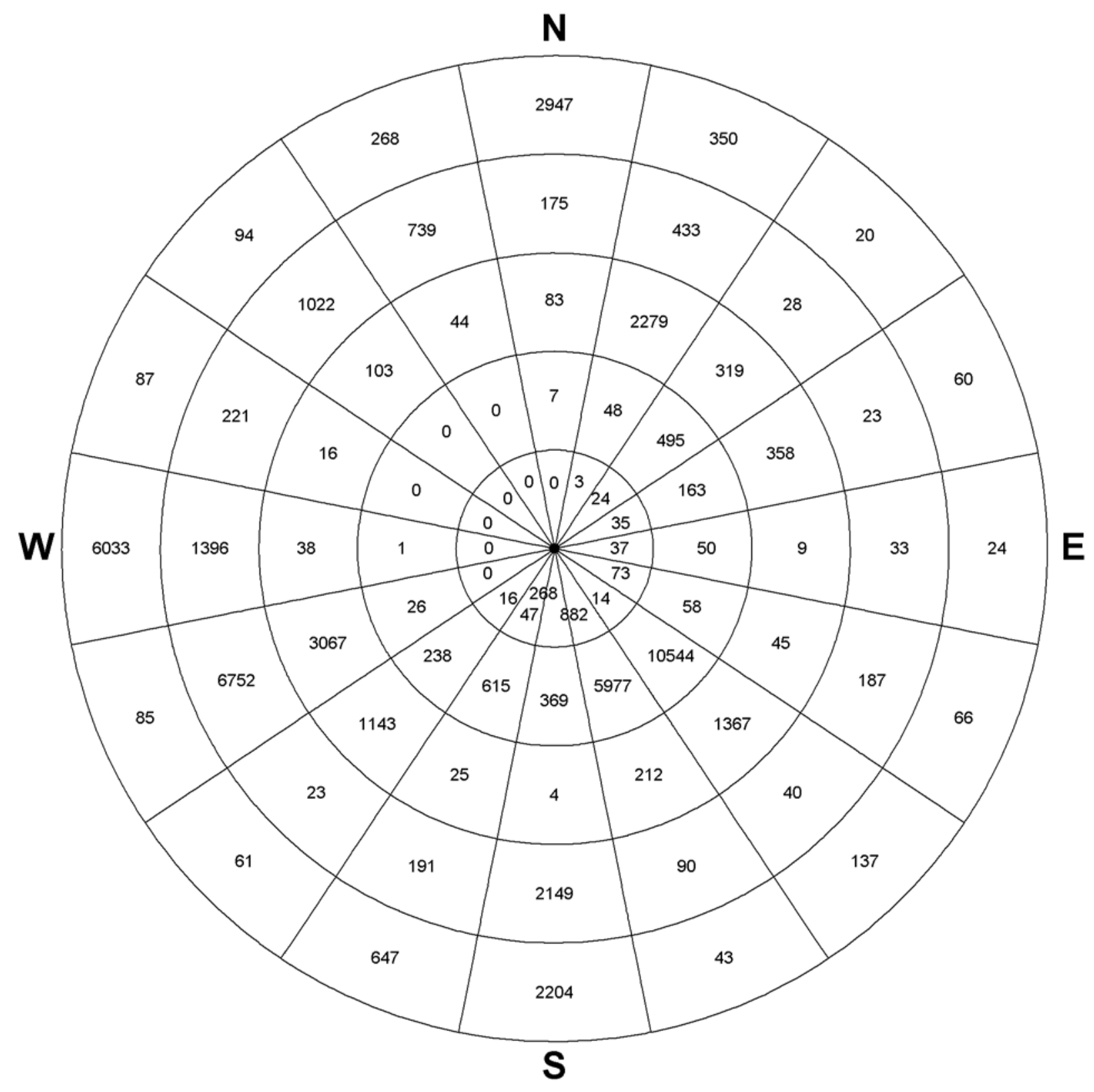

Figure 3.31. Map of Low-Income Population within $80 \mathrm{~km}(50 \mathrm{mi})$ of the Hanford 400 Area Meteorological Station, in 16-km (10-mi) Increments by Distance and Direction 
Table 3.22. Distribution of Total Resident Population within 80 km (50 mi) of the Hanford 400 Area Meteorological Station

\begin{tabular}{|c|c|c|c|c|c|c|c|c|c|c|c|c|c|c|c|c|c|}
\hline & \multicolumn{17}{|c|}{ Direction } \\
\hline $\begin{array}{l}\text { Distance } \\
\text { from } \\
\text { Reference } \\
\text { Point } \\
\text { (Miles) } \\
\end{array}$ & $\mathbf{N}$ & NNE & NE & ENE & $\mathbf{E}$ & ESE & SE & SSE & $\mathbf{S}$ & SSW & SW & WSW & $\mathbf{W}$ & WNW & NW & NNW & Total \\
\hline 0-1mi & 0 & 0 & 0 & 0 & 0 & 0 & 0 & 0 & 0 & 0 & 0 & 0 & 0 & 0 & 0 & 0 & $\mathbf{0}$ \\
\hline 1-2 & 0 & 0 & 0 & 0 & 0 & 0 & 0 & 0 & 0 & 0 & 0 & 0 & 0 & 0 & 0 & 0 & $\mathbf{0}$ \\
\hline $2-3$ & 0 & 0 & 0 & 0 & 0 & 0 & 0 & 0 & 0 & 0 & 0 & 0 & 0 & 0 & 0 & 0 & $\mathbf{0}$ \\
\hline $3-4$ & 0 & 4 & 0 & 0 & 0 & 0 & 0 & 0 & 0 & 0 & 0 & 0 & 0 & 0 & 0 & 0 & 4 \\
\hline $4-5$ & 0 & 0 & 0 & 0 & 2 & 0 & 0 & 0 & 0 & 52 & 43 & 0 & 0 & 0 & 0 & 0 & 97 \\
\hline 5-6 & 0 & 0 & 0 & 20 & 34 & 33 & 3 & 1 & 5 & 216 & 49 & 0 & 0 & 0 & 0 & 0 & 361 \\
\hline $6-7$ & 0 & 0 & 9 & 43 & 42 & 86 & 42 & 13 & 162 & 123 & 5 & 0 & 0 & 0 & 0 & 0 & 525 \\
\hline $7-8$ & 0 & 1 & 40 & 70 & 56 & 156 & 55 & 247 & 384 & 19 & 7 & 1 & 0 & 0 & 0 & 0 & 1,036 \\
\hline $8-9$ & 0 & 15 & 70 & 75 & 86 & 107 & 109 & 2,816 & 2,161 & 108 & 13 & 4 & 0 & 0 & 0 & 0 & 5,564 \\
\hline 9-10 & 0 & 10 & 87 & 90 & 93 & 223 & 125 & 8,978 & 1,913 & 386 & 195 & 5 & 0 & 0 & 0 & 0 & 12,105 \\
\hline 10-12 & 14 & 37 & 149 & 202 & 234 & 199 & 147 & 14,204 & 4,885 & 1,226 & 716 & 1 & 0 & 0 & 0 & 0 & 22,014 \\
\hline $12-14$ & 25 & 93 & 437 & 127 & 204 & 151 & 1,318 & 5,520 & 930 & 3,525 & 886 & 2 & 1 & 0 & 0 & 0 & 13,219 \\
\hline 14-16 & 26 & 140 & 1,009 & 188 & 93 & 88 & 3,814 & 14,390 & 976 & 173 & 262 & 10 & 4 & 0 & 0 & 0 & 21,173 \\
\hline 16-18 & 22 & 156 & 106 & 118 & 129 & 109 & 16,238 & 20,235 & 370 & 8 & 242 & 41 & 4 & 0 & 0 & 0 & 37,778 \\
\hline 18-20 & 19 & 82 & 90 & 402 & 42 & 31 & 26,408 & 14,582 & 477 & 14 & 350 & 163 & 2 & 0 & 0 & 0 & 42,662 \\
\hline $20-25$ & 115 & 896 & 229 & 73 & 48 & 332 & 13,382 & 6,838 & 47 & 71 & 3,736 & 1,065 & 8 & 48 & 27 & 8 & 26,923 \\
\hline $25-30$ & 600 & 9,960 & 1,734 & 1,631 & 54 & 326 & 1,516 & 26 & 5 & 101 & 4,246 & 13,106 & 204 & 57 & 449 & 197 & 34,212 \\
\hline 30-35 & 712 & 580 & 187 & 68 & 122 & 792 & 256 & 68 & 331 & 59 & 98 & 22,113 & 2,091 & 1,017 & 3,569 & 1,246 & 33,309 \\
\hline $35-40$ & 620 & 1,961 & 85 & 95 & 66 & 126 & 76 & 638 & 13,443 & 1,054 & 62 & 957 & 3,273 & 0 & 894 & 2,097 & 25,447 \\
\hline $40-50$ & 22,038 & 2,355 & 158 & 383 & 117 & 367 & 1,155 & 538 & 19,359 & 3,944 & 278 & 303 & 23,858 & 1,090 & 348 & 992 & 77,283 \\
\hline Total & 24,191 & 16,290 & 4,390 & 3,585 & 1,422 & 3,126 & 64,644 & 89,094 & 45,448 & 11,079 & 11,188 & 37,771 & 29,445 & 2,212 & 5,287 & 4,540 & 353,712 \\
\hline
\end{tabular}


Table 3.23. Distribution of Native American Population within 80 km (50 mi) of the Hanford 400 Area Meteorological Station

\begin{tabular}{|c|c|c|c|c|c|c|c|c|c|c|c|c|c|c|c|c|c|}
\hline \multirow[b]{2}{*}{$\begin{array}{l}\text { Distance } \\
\text { from } \\
\text { Reference } \\
\text { Point } \\
\text { (Miles) }\end{array}$} & \multicolumn{17}{|c|}{ Direction } \\
\hline & $\mathbf{N}$ & NNE & NE & ENE & $\mathbf{E}$ & ESE & SE & SSE & $\mathbf{S}$ & SSW & SW & WSW & $\mathbf{W}$ & WNW & NW & NNW & Total \\
\hline 0-1mi & 0 & 0 & 0 & 0 & 0 & 0 & 0 & 0 & 0 & 0 & 0 & 0 & 0 & 0 & 0 & 0 & 0 \\
\hline 1-2 & 0 & 0 & 0 & 0 & 0 & 0 & 0 & 0 & 0 & 0 & 0 & 0 & 0 & 0 & 0 & 0 & 0 \\
\hline $2-3$ & 0 & 0 & 0 & 0 & 0 & 0 & 0 & 0 & 0 & 0 & 0 & 0 & 0 & 0 & 0 & 0 & 0 \\
\hline $3-4$ & 0 & 0 & 0 & 0 & 0 & 0 & 0 & 0 & 0 & 0 & 0 & 0 & 0 & 0 & 0 & 0 & 0 \\
\hline $4-5$ & 0 & 0 & 0 & 0 & 0 & 0 & 0 & 0 & 0 & 0 & 0 & 0 & 0 & 0 & 0 & 0 & 0 \\
\hline 5-6 & 0 & 0 & 0 & 1 & 1 & 1 & 0 & 0 & 0 & 1 & 0 & 0 & 0 & 0 & 0 & 0 & 4 \\
\hline $6-7$ & 0 & 0 & 0 & 1 & 1 & 0 & 0 & 0 & 2 & 0 & 0 & 0 & 0 & 0 & 0 & 0 & 4 \\
\hline $7-8$ & 0 & 0 & 0 & 0 & 2 & 2 & 0 & 3 & 3 & 0 & 0 & 0 & 0 & 0 & 0 & 0 & 10 \\
\hline 8-9 & 0 & 0 & 0 & 0 & 3 & 1 & 0 & 23 & 20 & 0 & 0 & 0 & 0 & 0 & 0 & 0 & 47 \\
\hline 9-10 & 0 & 0 & 0 & 0 & 0 & 6 & 0 & 59 & 14 & 5 & 0 & 0 & 0 & 0 & 0 & 0 & 84 \\
\hline 10-12 & 0 & 0 & 1 & 0 & 0 & 1 & 0 & 145 & 12 & 13 & 1 & 0 & 0 & 0 & 0 & 0 & 173 \\
\hline $12-14$ & 0 & 0 & 0 & 0 & 0 & 0 & 11 & 26 & 5 & 41 & 8 & 1 & 0 & 0 & 0 & 0 & 92 \\
\hline 14-16 & 0 & 0 & 6 & 1 & 0 & 0 & 20 & 93 & 5 & 1 & 1 & 4 & 0 & 0 & 0 & 0 & 131 \\
\hline 16-18 & 0 & 0 & 0 & 0 & 0 & 0 & 119 & 142 & 1 & 0 & 3 & 0 & 0 & 0 & 0 & 0 & 265 \\
\hline $18-20$ & 0 & 0 & 0 & 4 & 0 & 0 & 293 & 115 & 8 & 0 & 0 & 5 & 0 & 0 & 0 & 0 & 425 \\
\hline 20-25 & 2 & 2 & 1 & 0 & 0 & 1 & 136 & 51 & 0 & 0 & 16 & 4 & 0 & 0 & 0 & 0 & 213 \\
\hline 25-30 & 8 & 76 & 17 & 16 & 0 & 5 & 7 & 0 & 0 & 0 & 36 & 113 & 1 & 0 & 5 & 0 & 284 \\
\hline $30-35$ & 5 & 1 & 1 & 0 & 0 & 14 & 2 & 0 & 3 & 0 & 0 & 168 & 17 & 2 & 21 & 6 & 240 \\
\hline 35-40 & 1 & 11 & 0 & 0 & 0 & 1 & 0 & 7 & 138 & 24 & 6 & 125 & 59 & 0 & 4 & 10 & 386 \\
\hline 40-50 & 209 & 20 & 0 & 2 & 0 & 0 & 7 & 2 & 174 & 79 & 7 & 142 & 2,283 & 14 & 4 & 3 & 2,946 \\
\hline Total & 225 & 110 & 26 & 25 & 7 & 32 & 595 & 666 & 385 & 164 & 78 & 562 & 2,360 & 16 & 34 & 19 & 5,304 \\
\hline
\end{tabular}


Table 3.24. Distribution of Hispanic and Latino Population within 80 km (50 mi) of the Hanford 400 Area Meteorological Station

\begin{tabular}{|c|c|c|c|c|c|c|c|c|c|c|c|c|c|c|c|c|c|}
\hline & \multicolumn{17}{|c|}{ Direction } \\
\hline $\begin{array}{l}\text { Distance } \\
\text { from } \\
\text { Reference } \\
\text { Point } \\
\text { (Miles) } \\
\end{array}$ & $\mathbf{N}$ & NNE & NE & ENE & $\mathbf{E}$ & ESE & SE & SSE & $\mathbf{S}$ & SSW & SW & WSW & $\mathbf{W}$ & WNW & NW & NNW & Total \\
\hline 0-1mi & 0 & 0 & 0 & 0 & 0 & 0 & 0 & 0 & 0 & 0 & 0 & 0 & 0 & 0 & 0 & 0 & $\mathbf{0}$ \\
\hline 1-2 & 0 & 0 & 0 & 0 & 0 & 0 & 0 & 0 & 0 & 0 & 0 & 0 & 0 & 0 & 0 & 0 & $\mathbf{0}$ \\
\hline $2-3$ & 0 & 0 & 0 & 0 & 0 & 0 & 0 & 0 & 0 & 0 & 0 & 0 & 0 & 0 & 0 & 0 & $\mathbf{0}$ \\
\hline $3-4$ & 0 & 0 & 0 & 0 & 0 & 0 & 0 & 0 & 0 & 0 & 0 & 0 & 0 & 0 & 0 & 0 & $\mathbf{0}$ \\
\hline 4-5 & 0 & 0 & 0 & 0 & 1 & 0 & 0 & 0 & 0 & 1 & 1 & 0 & 0 & 0 & 0 & 0 & 3 \\
\hline 5-6 & 0 & 0 & 0 & 10 & 17 & 13 & 1 & 0 & 0 & 2 & 1 & 0 & 0 & 0 & 0 & 0 & 44 \\
\hline $6-7$ & 0 & 0 & 3 & 21 & 18 & 29 & 3 & 0 & 7 & 1 & 2 & 0 & 0 & 0 & 0 & 0 & 84 \\
\hline $7-8$ & 0 & 0 & 15 & 38 & 25 & 20 & 0 & 7 & 16 & 2 & 2 & 0 & 0 & 0 & 0 & 0 & 125 \\
\hline $8-9$ & 0 & 3 & 33 & 30 & 37 & 19 & 10 & 103 & 101 & 9 & 2 & 1 & 0 & 0 & 0 & 0 & 348 \\
\hline 9-10 & 0 & 1 & 50 & 29 & 24 & 43 & 6 & 423 & 107 & 16 & 4 & 2 & 0 & 0 & 0 & 0 & 705 \\
\hline 10-12 & 2 & 3 & 67 & 119 & 74 & 61 & 6 & 697 & 206 & 94 & 78 & 0 & 0 & 0 & 0 & 0 & 1,407 \\
\hline $12-14$ & 3 & 15 & 268 & 40 & 78 & 26 & 304 & 307 & 51 & 637 & 134 & 1 & 0 & 0 & 0 & 0 & 1,864 \\
\hline 14-16 & 6 & 28 & 693 & 69 & 4 & 16 & 608 & 847 & 41 & 28 & 45 & 4 & 0 & 0 & 0 & 0 & 2,389 \\
\hline 16-18 & 7 & 63 & 15 & 40 & 34 & 14 & 7,308 & 2,127 & 27 & 0 & 116 & 20 & 0 & 0 & 0 & 0 & 9,771 \\
\hline $18-20$ & 0 & 15 & 14 & 233 & 12 & 8 & 14,080 & 1,692 & 19 & 0 & 113 & 92 & 1 & 0 & 0 & 0 & 16,279 \\
\hline 20-25 & 58 & 296 & 69 & 28 & 11 & 94 & 1,826 & 366 & 4 & 4 & 1,218 & 612 & 1 & 14 & 15 & 6 & 4,622 \\
\hline $25-30$ & 314 & 6,547 & 441 & 851 & 6 & 48 & 138 & 6 & 0 & 15 & 1,141 & 7,906 & 77 & 11 & 267 & 116 & 17,884 \\
\hline $30-35$ & 334 & 335 & 53 & 9 & 30 & 731 & 49 & 45 & 89 & 20 & 34 & 15,245 & 907 & 502 & 2,934 & 807 & 22,124 \\
\hline $35-40$ & 101 & 1,153 & 18 & 3 & 17 & 70 & 14 & 64 & 3,678 & 189 & 24 & 512 & 1,703 & 0 & 652 & 1,347 & 9,545 \\
\hline $40-50$ & 4,906 & 976 & 22 & 32 & 8 & 71 & 191 & 30 & 4,352 & 1,724 & 138 & 41 & 12,788 & 145 & 148 & 321 & 25,893 \\
\hline Total & 5,731 & 9,435 & 1,761 & 1,552 & 396 & 1,263 & 24,544 & 6,714 & 8,698 & 2,742 & 3,053 & 24,436 & 15,477 & 672 & 4,016 & 2,597 & 113,087 \\
\hline
\end{tabular}


Table 3.25. Distribution of Total Minority Population within $80 \mathrm{~km}(50 \mathrm{mi})$ of the Hanford 400 Area Meteorological Station

\begin{tabular}{|c|c|c|c|c|c|c|c|c|c|c|c|c|c|c|c|c|c|}
\hline & \multicolumn{17}{|c|}{ Direction } \\
\hline $\begin{array}{l}\text { Distance } \\
\text { from } \\
\text { Reference } \\
\text { Point } \\
\text { (Miles) } \\
\end{array}$ & $\mathbf{N}$ & NNE & NE & ENE & $\mathbf{E}$ & ESE & SE & SSE & $\mathbf{S}$ & SSW & SW & WSW & $\mathbf{W}$ & WNW & NW & NNW & Total \\
\hline 0-1mi & 0 & 0 & 0 & 0 & 0 & 0 & 0 & 0 & 0 & 0 & 0 & 0 & 0 & 0 & 0 & 0 & $\mathbf{0}$ \\
\hline 1-2 & 0 & 0 & 0 & 0 & 0 & 0 & 0 & 0 & 0 & 0 & 0 & 0 & 0 & 0 & 0 & 0 & $\mathbf{0}$ \\
\hline $2-3$ & 0 & 0 & 0 & 0 & 0 & 0 & 0 & 0 & 0 & 0 & 0 & 0 & 0 & 0 & 0 & 0 & $\mathbf{0}$ \\
\hline $3-4$ & 0 & 2 & 0 & 0 & 0 & 0 & 0 & 0 & 0 & 0 & 0 & 0 & 0 & 0 & 0 & 0 & 2 \\
\hline $4-5$ & 0 & 0 & 0 & 0 & 1 & 0 & 0 & 0 & 0 & 1 & 2 & 0 & 0 & 0 & 0 & 0 & 4 \\
\hline $5-6$ & 0 & 0 & 0 & 11 & 19 & 14 & 1 & 0 & 0 & 7 & 2 & 0 & 0 & 0 & 0 & 0 & 54 \\
\hline $6-7$ & 0 & 0 & 3 & 23 & 20 & 30 & 4 & 3 & 30 & 4 & 2 & 0 & 0 & 0 & 0 & 0 & 119 \\
\hline $7-8$ & 0 & 0 & 15 & 38 & 27 & 22 & 0 & 46 & 52 & 3 & 2 & 0 & 0 & 0 & 0 & 0 & 205 \\
\hline $8-9$ & 0 & 4 & 35 & 30 & 40 & 23 & 11 & 436 & 193 & 11 & 3 & 1 & 0 & 0 & 0 & 0 & 787 \\
\hline 9-10 & 0 & 2 & 51 & 30 & 26 & 55 & 7 & 1,235 & 202 & 25 & 5 & 2 & 0 & 0 & 0 & 0 & 1,640 \\
\hline 10-12 & 2 & 4 & 70 & 119 & 76 & 66 & 11 & 1,631 & 413 & 132 & 87 & 0 & 0 & 0 & 0 & 0 & 2,611 \\
\hline $12-14$ & 3 & 17 & 275 & 44 & 80 & 32 & 381 & 740 & 88 & 783 & 171 & 1 & 0 & 0 & 0 & 0 & 2,615 \\
\hline 14-16 & 6 & 30 & 709 & 69 & 5 & 18 & 831 & 1,769 & 77 & 32 & 46 & 4 & 0 & 0 & 0 & 0 & 3,596 \\
\hline 16-18 & 7 & 66 & 21 & 40 & 37 & 14 & 8,466 & 3,395 & 44 & 0 & 126 & 21 & 0 & 0 & 0 & 0 & 12,237 \\
\hline $18-20$ & 1 & 16 & 17 & 241 & 13 & 8 & 15,809 & 2,505 & 35 & 0 & 115 & 99 & 1 & 0 & 0 & 0 & 18,860 \\
\hline 20-25 & 63 & 302 & 73 & 28 & 11 & 97 & 2,318 & 669 & 5 & 6 & 1,309 & 635 & 1 & 14 & 16 & 6 & 5,553 \\
\hline $25-30$ & 331 & 6,714 & 634 & 977 & 9 & 59 & 175 & 6 & 0 & 18 & 1,240 & 8,204 & 85 & 15 & 282 & 117 & 18,866 \\
\hline 30-35 & 345 & 348 & 60 & 10 & 45 & 738 & 51 & 46 & 108 & 20 & 34 & 15,740 & 955 & 527 & 2,990 & 833 & 22,850 \\
\hline $35-40$ & 116 & 1,179 & 25 & 4 & 20 & 77 & 15 & 84 & 4,143 & 249 & 29 & 661 & 1,816 & 0 & 655 & 1,381 & 10,454 \\
\hline $40-50$ & 6,132 & 1,029 & 34 & 43 & 11 & 77 & 216 & 42 & 5,175 & 1,890 & 152 & 195 & 15,530 & 188 & 154 & 352 & 31,220 \\
\hline Total & 7,006 & 9,713 & 2,022 & 1,707 & 440 & 1,330 & 28,296 & 12,607 & 10,565 & 3,181 & 3,325 & 25,563 & 18,388 & 744 & 4,097 & 2,689 & 131,673 \\
\hline
\end{tabular}


Table 3.26. Distribution of Low-Income Population within 80 km (50 mi) of the Hanford 400 Area Meteorological Station

\begin{tabular}{|c|c|c|c|c|c|c|c|c|c|c|c|c|c|c|c|c|c|}
\hline & \multicolumn{17}{|c|}{ Direction } \\
\hline $\begin{array}{l}\text { Distance } \\
\text { from } \\
\text { Reference } \\
\text { Point } \\
\text { (Miles) }\end{array}$ & $\mathbf{N}$ & NNE & NE & ENE & $\mathbf{E}$ & ESE & SE & SSE & $\mathbf{S}$ & SSW & SW & WSW & $\mathbf{W}$ & WNW & NW & NNW & Total \\
\hline $0-1 \mathrm{mi}$ & 0 & 0 & 0 & 0 & 0 & 0 & 0 & 0 & 0 & 0 & 0 & 0 & 0 & 0 & 0 & 0 & $\mathbf{0}$ \\
\hline $1-2$ & 0 & 0 & 0 & 0 & 0 & 0 & 0 & 0 & 0 & 0 & 0 & 0 & 0 & 0 & 0 & 0 & 0 \\
\hline $2-3$ & 0 & 0 & 0 & 0 & 0 & 0 & 0 & 0 & 0 & 0 & 0 & 0 & 0 & 0 & 0 & 0 & $\mathbf{0}$ \\
\hline $3-4$ & 0 & 0 & 0 & 0 & 0 & 0 & 0 & 0 & 0 & 0 & 0 & 0 & 0 & 0 & 0 & 0 & $\mathbf{0}$ \\
\hline $4-5$ & 0 & 0 & 0 & 0 & 0 & 0 & 0 & 0 & 0 & 2 & 2 & 0 & 0 & 0 & 0 & 0 & 4 \\
\hline $5-6$ & 0 & 0 & 0 & 2 & 4 & 4 & 0 & 0 & 0 & 10 & 2 & 0 & 0 & 0 & 0 & 0 & 22 \\
\hline 6-7 & 0 & 0 & 1 & 5 & 5 & 10 & 5 & 1 & 7 & 6 & 0 & 0 & 0 & 0 & 0 & 0 & 40 \\
\hline $7-8$ & 0 & 0 & 5 & 8 & 7 & 19 & 3 & 8 & 20 & 1 & 0 & 0 & 0 & 0 & 0 & 0 & 71 \\
\hline $8-9$ & 0 & 2 & 8 & 9 & 10 & 13 & 2 & 130 & 120 & 5 & 0 & 0 & 0 & 0 & 0 & 0 & 299 \\
\hline 9-10 & 0 & 1 & 10 & 11 & 11 & 27 & 4 & 743 & 121 & 23 & 12 & 0 & 0 & 0 & 0 & 0 & 963 \\
\hline $10-12$ & 1 & 2 & 27 & 24 & 27 & 24 & 2 & 1,452 & 221 & 81 & 56 & 0 & 0 & 0 & 0 & 0 & 1,917 \\
\hline $12-14$ & 2 & 6 & 117 & 11 & 8 & 14 & 14 & 361 & 99 & 525 & 76 & 0 & 0 & 0 & 0 & 0 & 1,233 \\
\hline $14-16$ & 2 & 9 & 294 & 10 & 4 & 7 & 67 & 822 & 26 & 8 & 21 & 1 & 1 & 0 & 0 & 0 & 1,272 \\
\hline 16-18 & 1 & 16 & 31 & 15 & 7 & 10 & 3,451 & 2,085 & 10 & 0 & 27 & 5 & 0 & 0 & 0 & 0 & 5,658 \\
\hline $18-20$ & 1 & 15 & 26 & 103 & 4 & 3 & 7,010 & 1,257 & 13 & 1 & 58 & 20 & 0 & 0 & 0 & 0 & 8,511 \\
\hline $20-25$ & 7 & 126 & 60 & 11 & 4 & 29 & 1,286 & 210 & 3 & 9 & 659 & 186 & 1 & 6 & 6 & 2 & 2,605 \\
\hline $25-30$ & 76 & 2,153 & 259 & 347 & 5 & 16 & 81 & 2 & 1 & 16 & 484 & 2,881 & 37 & 10 & 97 & 42 & 6,507 \\
\hline 30-35 & 101 & 71 & 18 & 9 & 20 & 162 & 31 & 9 & 54 & 9 & 11 & 6,478 & 457 & 221 & 775 & 251 & $\mathbf{8 , 6 7 7}$ \\
\hline $35-40$ & 74 & 362 & 10 & 14 & 13 & 25 & 9 & 81 & 2,095 & 182 & 12 & 274 & 939 & 0 & 247 & 488 & 4,825 \\
\hline $40-50$ & 2,947 & 350 & 20 & 60 & 24 & 66 & 137 & 43 & 2,204 & 647 & 61 & 85 & 6,033 & 87 & 94 & 268 & 13,126 \\
\hline Total & 3,212 & 3,113 & 886 & 639 & 153 & 429 & 12,102 & 7,204 & 4,994 & 1,525 & 1,481 & 9,930 & 7,468 & 324 & 1,219 & 1,051 & 55,730 \\
\hline
\end{tabular}




\subsection{References}

42 USC 4321 et seq. National Environmental Policy Act (NEPA) of 1969, as amended. Online at: http://www4.law.cornell.edu

59 FR 7629. Executive Order 12898, "Federal Actions to Address Environmental Justice in Minority and Low-Income Populations.” Federal Register. Vol. 59, No. 32. February 16, 1994.

Beck, D.M , M.J Scott, M.D. Davis, S.F. Shindle, B.A. Napier, A.G. Thurman, D.B. Pittenger, and N.C. Batishko. 1991. Hanford Area 1990 Population and 50-Year Projections. PNL-7803. Pacific Northwest Laboratory, Richland, Washington.

Census: See U.S. Census Bureau.

Council on Environmental Quality (CEQ). 1997. Environmental Justice: Guidance Under the National Environmental Policy Act. Executive Office of the President, Washington, D.C.

DOE. 1993. Radiation Protection of the Public and the Environment. DOE Order 5400.5, U.S. Department of Energy, Washington, D.C. Online at: http://www.directives.doe.gov

Sommer, D.J., R.G. Rau, and D.C. Robinson. 1981. Population Estimates for the Areas Within a 50-mile Radius of Four Reference Points on the Hanford Site. PNL-4010. Pacific Northwest Laboratory, Richland, Washington.

U.S. Census Bureau (Census). 1991. Table DP-1. General Population and Housing Characteristics: 1990. Data Set: 1990 Summary Tape File 1 (STF 1) 100-Percent Data. (Website: http://factfinder.census.gov/servlet/QTGeoSearchByListServlet?ds name=DEC 1990 STF1 \& lang=en) Accessed September 8, 2003.

U.S. Census Bureau (Census). 2001a. Census 2000 Redistricting Data (P.L. 94-171) Summary File Oregon. C1-D00-PLF1-07-OR1. U.S. Census Bureau, Washington D.C.

U.S. Census Bureau (Census). 2001b. Census 2000 Redistricting Data (P.L. 94-171) Summary File Washington. C1-D00-PLF1-07-WA1. U.S. Census Bureau, Washington D.C.

U.S. Census Bureau (Census). 2001c. Redistricting Census 2000 Tiger/Line Files. (Website: http://www.esri.com/data/download/census2000_tigerline/index.html). U.S. Census Bureau, Washington D.C.

U.S. Census Bureau (Census). 2001d. Table DP-1. Profile of General Demographic Characteristics: 2000. Data Set: Census 2000 Summary File 1 (SF 1) 100-Percent Data. (Website: http://factfinder.census.gov/servlet/QTGeoSearchByListServlet?ds name=DEC 2000 SF1_U\& lang=en) Accessed September 8, 2003.

U.S. Census Bureau (Census). 2002a. Census 2000 Summary File 3 - Oregon. (Website: http://www2.census.gov/census_2000/datasets/Summary_File_3/Oregon/). U.S. Census Bureau, Washington D.C.

U.S. Census Bureau (Census). 2002b. Census 2000 Summary File 3 - Washington. (Website: http://www2.census.gov/census_2000/datasets/Summary_File_3/Washington/). U.S. Census Bureau, Washington D.C.

U.S. Census Bureau (Census). 2003a. Glossary - Definition and Explanations-decennial census terms. (Website: http://www.census.gov/main/www/glossary.html. Last revised February 11, 2003.) Accessed September 5, 2003. 
U.S. Census Bureau (Census). 2003b. Poverty 1999. Census 2000 Brief. C2KBR-19. Issued May 2003. (Website: http://www.census.gov/prod/2003pubs/c2kbr-19.pdf.) Accessed September 8, 2003.

U.S. Nuclear Regulatory Commission (NRC). 1975. Standard Format and Content of Safety Analysis Reports for Fuel Reprocessing Plants. Regulatory Guide 3.26, Washington, D.C.

U.S. Nuclear Regulatory Commission (NRC). 1988. Standard Format and Content of a License Application for a Low-Level Radioactive Waste Disposal Facility. NUREG-1199, Revision 1, Washington D.C. 